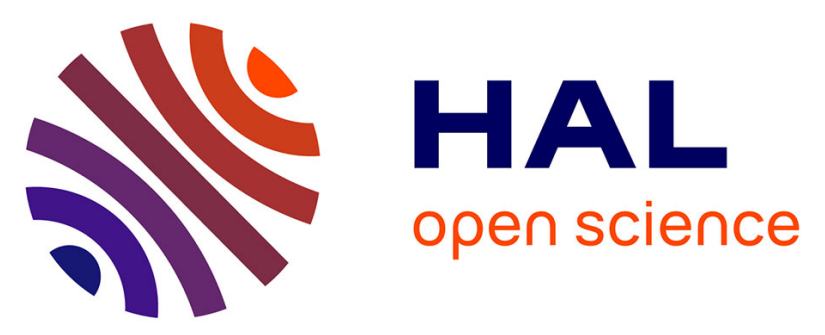

\title{
Un ancien lac au pied de l'oppidum de Gergovie (Puy-de-Dôme) : interactions sociétés-milieux dans le bassin de Sarliève à l'Holocène
}

Frédéric Trément, Jacqueline Argant, Jean-Gabriel Bréhéret, Manon Cabanis, Bertrand Dousteyssier, Agathe Fourmont, Gabriel Fournier, René Liabeuf, Gilles Loison, José Antonio López Sáez, et al.

\section{To cite this version:}

Frédéric Trément, Jacqueline Argant, Jean-Gabriel Bréhéret, Manon Cabanis, Bertrand Dousteyssier, et al.. Un ancien lac au pied de l'oppidum de Gergovie (Puy-de-Dôme) : interactions sociétés-milieux dans le bassin de Sarliève à l'Holocène. Gallia - Archéologie de la France antique, 2007, 64, pp.289-351. 10.3406/galia.2007.3314 . hal-00351025

\section{HAL Id: hal-00351025 \\ https://hal.science/hal-00351025}

Submitted on 11 Feb 2013

HAL is a multi-disciplinary open access archive for the deposit and dissemination of scientific research documents, whether they are published or not. The documents may come from teaching and research institutions in France or abroad, or from public or private research centers.
L'archive ouverte pluridisciplinaire HAL, est destinée au dépôt et à la diffusion de documents scientifiques de niveau recherche, publiés ou non, émanant des établissements d'enseignement et de recherche français ou étrangers, des laboratoires publics ou privés.

\section{(1) (1) $\$$}

Distributed under a Creative Commons Attribution - NonCommercial - NoDerivatives 44.0 


\title{
UN ANCIEN LAC AU PIED DE L'OPPIDUM DE GERGOVIE (PUY-DE-DÔME)
}

\section{Interactions sociétés-milieux dans le bassin de Sarliève à l'Holocène}

\author{
Sous la direction de Frédéric TRÉMENT* \\ avec les contributions de Jacqueline ARGANT, Jean-Gabriel BRÉHÉRET, \\ Manon CABAnis, Bertrand Dousteyssier, Agathe Fourmont, Gabriel FourniER, \\ René LiAbeUf, Gilles LoISOn, José Antonio LóPEz SÁEZ, Jean-Jacques MACAIRE, \\ Philippe Marinval, Christine Mennessier-Jouannet, Pierre-Yves Milcent, \\ Béatrice PRAT, Yannick RIALLAND, Gérard VERNET
}

\begin{abstract}
Mots-clés. Limagne, Arvernes, paléoenvironnement, anthroposystème, habitat rural, marais, zone humide, drainage, climat. Résumé. Les recherches conduites depuis 1997 dans le bassin de Sarliève, au pied de l'oppidum de Gergovie (Puy-de-Dôme), visent à appréhender, dans une perspective systémique et interdisciplinaire, les interactions sociétés-milieux dans la longue durée, à l'échelle d'un bassin-versant de taille moyenne localisé dans une zone de première importance sur le plan historique. Trois axes de recherche ont été définis :

- la caractérisation de l'évolution du milieu à l'échelle du bassin-versant dans ses différentes composantes biophysiques ;

- la caractérisation de l'histoire de l'occupation du sol et de l'exploitation du milieu par les populations successives établies dans le bassin;

- la caractérisation de l'impact de la pression anthropique sur le milieu (couverture végétale, dynamiques hydrosédimentaires). Les résultats obtenus fournissent les éléments d'un modèle socio-environnemental qui renouvelle celui élaboré pour la Grande Limagne dans les années 1970-1980. Une succession de cycles agraires est clairement mise en évidence. Ceux-ci sont liés à des phases de forte pression humaine identifiées au Néolithique moyen, au Bronze ancien, au Bronze final, au premier âge du Fer, à La Tène finale, au Haut-Empire, au Moyen Âge et à l'époque moderne. Ces cycles sont étroitement corrélés avec l'évolution de la végétation et la dynamique sédimentaire, mais également avec les variations verticales du plan d'eau. Des phases de transgression particulièrement amples et contraignantes sont mises en évidence durant l'âge du Bronze et le Moyen Âge. Le bas niveau du paléolac durant le second âge du Fer et le Haut-Empire est, en revanche, propice à l'occupation des bordures et même du fond de la cuvette, qui est complètement asséchée grâce à un système de drainage aménagé aux environs du changement d'ère.

Un seuil quantitatif très net est franchi dans la première moitié du II ${ }^{e}$ s. av. J.-C., avec la forte densification de l'habitat dans tous les types d'unités physiques. Les données archéologiques et paléoenvironnementales traduisent la mise en valeur méthodique des sols les plus fertiles, dans un contexte de croissance économique et démographique continue qui coïncide avec l'apparition de vastes agglomérations interprétées comme des "places centrales " (agglomération de la Grande Borne à Aulnat, oppida de Corent à VeyreMonton, Gergovie à La Roche-Blanche et Gondole au Cendre). Cette tendance se poursuit durant les deux premiers siècles de notre ère, avec la généralisation de l'agrosystème à l'ensemble du bassin, quels que soient les types de reliefs, dans le cadre de l'économie domaniale qui se développe à la suite de la fondation d'Augustonemetum, nouvelle capitale de la cité arverne. La fin de l'Antiquité et le haut Moyen Âge voient le retour du marais à la faveur d'un climat plus humide et, surtout, de profonds changements dans les modalités de gestion du milieu. Un véritable lac est attesté par les textes médiévaux et par l'iconographie moderne. Il est définitivement asséché au début du XVII ${ }^{e}$.
\end{abstract}

* Les adresses des auteurs se trouvent en fin d'article, p. 351. 
Keywords. Limagne, Arverni, palaeoenvironment, anthroposystem, rural settlement, marsh, wet-land, draining, climate. Abstract. The aim of the researches conducted since 1997 in the basin of Sarliève, at the foot of the oppidum of Gergovia (Puy-de-Dôme), is to detect in a systemic and interdisciplinary perspective the longlasting interactions societies/environment on the scale of a mediumsized basin located in a major area from an historical point of view. Three axes have been defined:

- the characterization of the evolution of the environment on the scale of the basin inside its various biophysical components;

- the characterization of the history of land occcupation and of the exploitation of environment by successive populations settled in the basin;

- the characterization of the impact of human pressure on the environment (plant cover, hydro-sedimentary dynamics).

Results provide elements of a new socioenvironmental model after the model completed in Limagne in the 1970'-1980'. A succession of land cycles has been detected and connected to phases of strong human pressure identified for Middle Neolithic, Early and Late Bronze Age, Early Iron Age, Early Empire, Middle Age and Modern period. These cycles are tightly connected with vegetation evolution and sedimentary dynamics but also with vertical variations of the lake level. Some phases of encroachment, particularly wide and constraining, have been revealed for Bronze Age and Middle Age. The low level of the lake during the Late Iron Age and the Early Empire in the other hand is favourable to the occupation of the edges and even at the bottom of the basin which is entirely drained due to a draining system at the turn of our era.

A definite quantitative step, during the first half of the $2^{\text {nd }}$ century B.C., is represented by the high densification of the populated area in all kinds of physical units. Archaeological and environmental datas reveal a methodic exploitation of the most fertile lands in a context of continuous economic and demographic environment which coincides with the birth of towns identified as "central places" (such as la Grande Borne at Aulnat and the oppida of Corent at Veyre-Monton, Gergovia at La Roche-Blanche and Gondole at Le Cendre). This tendency goes on during the first two centuries A.D., with the generalization of agrosystem to the whole basin, for any kind of relief, in the context of economic estates growing since the foundation of Augustonemetum, the new capital of the Arverni. The return of marsh in Late Antiquity and Early Middle Age is due to a more humid climate and to deep changes in the methods of environmental management. A real lake is attested by medieval sources and modern iconography and is definitely dried out at the beginning of the $17^{\text {th }}$ century.

\section{Translation: Isabelle FAUDUET}

Schlüsselwörter. Limagne, Arverner, Umwelt im Holozän, Anthroposystem, ländliche Siedlung, Sumpf, Feuchtzone, Entwässerung, Klima.

Zusammenfassung. Ziel der seit 1997 im Becken von Sarliève, am Fuß des Oppidums von Gergovia (Puy-de-Dôme) durchgeführten Studien ist es, die wechselseitigen Beziehungen zwischen Mensch und Umwelt über einen langen Zeitraum aus einem systemischen und interdisziplinären Blickwinkel zu betrachten. Die Untersuchungen beziehen sich auf ein Niederschlagsgebiet mittlerer Größe in einer Region von eminenter geschichtlicher Bedeutung. Drei Untersuchungsachsen wurden definiert:

- die Analyse der Entwicklung der Umwelt im Bereich dieses Niederschlagsgebietes in Hinsicht auf seine unterschiedlichen biophysikalischen Komponenten;

- die Analyse der Geschichte der Flächennutzung und der Bewirtschaftung des Beckens durch die sich ablösenden Bevölkerungsgruppen;

- die Analyse der durch den Menschen hervorgerufenen Umweltbelastung (Pflanzendecke, hydrosedimentäre Kräfte).

Die Ergebnisse dieser Forschungen liefern die Elemente für die Erstellung eines sozialökologischen Modells, das das von 1970-1980 für die Grande Limagne ausgearbeitete Modell ablöst. Eine Abfolge von Agrarzyklen ist deutlich erkennbar. Diese stehen im Zusammenhang mit Phasen starker menschlicher Einflussnahme, die für das Mittelneolithikum, die Früh-und Spätbronzezeit, die ältere Eisenzeit, die späte Latène- und frühe Kaiserzeit, das Mittelalter und die Neuzeit erkannt wurden. Diese Zyklen sind eng verknüpft nicht nur mit der Entwicklung der Pflanzen und der Sedimentierungsdynamik, sondern auch mit den Schwankungen des Wasserstandes. In der Bronzezeit und im Mittelalter werden Transgressionsphasen mit besonders umfangreichen und einengenden Auswirkungen nachgewiesen.

Der niedrige Pegel des Paläosees in der jüngeren Eisenzeit und der frühen Kaiserzeit begünstigt dagegen die Besiedlung der Uferregionen und sogar der Sohle des Beckens, das um die Zeitenwende durch ein Entwässerungssystem vollständig trocken gelegt wird.

In der ersten Hälfte des 2. Jh. v. Chr. wird mit der starken Intensivierung der Siedlungstätigkeit in sämtlichen topographischen Kontexten eine deutliche quantitative Schwelle überschritten. Die Ergebnisse der archäologischen und paläoökologischen Untersuchungen lassen die methodische Erschließung der fruchtbarsten Böden im Zusammenhang mit einem stetigen wirtschaftlichen und demographischen Wachstum erkennen, das mit dem Bau großer als „zentrale Plätze“ interpretierter Siedlungen (La Grande Borne in Aulnat, Oppida von Corent, Gergovia und Gondole) zusammenfällt. In den ersten beiden Jahrhunderten unseres Zeitalters setzt sich diese Tendenz im Rahmen der Ökonomie der villae, die sich infolge der Gründung von Augustonemetum, der neuen Hauptstadt der avernischen civitas herausbildet, mit der von den Geländeformen unabhängigen Ausweitung der landwirtschaftlichen Nutzung auf das gesamte Becken fort. In der Spätantike und im frühen Mittelalter gewinnt der Sumpf in einem feuchteren Klima wieder an Boden, besonders durch die grundlegenden Veränderungen in der Art und Weise das Gebiet zu nutzen. Die mittelalterlichen Texte und die neuzeitliche Ikonographie bezeugen die Existenz eines Sees, der zu Beginn des 17. Jh. schließlich endgültig trockengelegt wird.

Übersetzung: Isa ODENHARDT-DONVEZ 


\section{CONTEXTE DES RECHERCHES, PROBLÉMATIQUES ET MÉTHODES}

\section{CADRE DES RECHERCHES}

Depuis le $\mathrm{XVI}^{\mathrm{e}} \mathrm{s}$. et la Description de la Limagne d'Auvergne en forme de dialogue du florentin Gabriel Syméoni (1561), l'ancien lac de Sarliève est étroitement associé à l'oppidum de Gergovie, au pied duquel il s'étend. Les érudits ont posé très tôt la question de son origine et de son évolution, avec l'idée de reconstituer le paysage qui fut le siège de la fameuse bataille de 52 av. J.-C. Certains auteurs, à la suite de Jean Savaron (1599), ont imaginé que la résidence d'Avitacum, dont Sidoine Apollinaire avait hérité de son beau-père, l'empereur Avitus, se trouvait au bord du lac. Dans son Vercingétorix, Camille Jullian (1901, p. 197) imaginait que « le lac de Sarlièves (sic) était peut-être le dépôt inviolable des trésors voués aux dieux ».

Plus prosaïquement, à l'image de notre siècle, les recherches interdisciplinaires conduites depuis 1997 visent à appréhender, dans une perspective systémique, les interactions sociétés-milieux dans la longue durée, à l'échelle d'un bassin-versant de taille moyenne situé dans une zone de première importance (fig. 1). Le bassin de Sarliève se localise en effet à proximité des grands centres de pouvoir arvernes: le complexe de la Grande Borne à Aulnat, les oppida de Corent, Gondole et Gergovie, le chef-lieu de cité : Augustonemetum. Deux axes routiers majeurs se rejoignent en bordure nord du paléolac dès l'Antiquité : la voie de Lyon à Saintes (Charente-Maritime), et celle qui reliait ClermontFerrand à la Méditerranée. Cette dépression asséchée au début du XVII ${ }^{\mathrm{e}} \mathrm{s}$. constitue également un excellent enregistreur des dynamiques environnementales holocènes. C'est, en particulier, l'un des rares pièges à sédiments de la Limagne propice à la conservation des pollens. Enfin, ce bassin a fait l'objet, au cours des dernières années, de nombreuses opérations d'archéologie préventive, dont plusieurs menées en bordure ou à l'intérieur du paléolac.

Les recherches ont été conduites, entre 1997 et 2001, dans le cadre du programme interdisciplinaire de recherche «Environnement, Vie et Société» du CNRS, intitulé «Interactions hommes/milieux dans le bassin-versant de la Loire (fin du Tardiglaciaire-Époque industrielle) ». Elles se sont prolongées, à partir de 2001, au sein de la "ZoneAtelier Loire " ${ }^{1}$. Elles ont également pris place, à partir

1. F. Trément a assuré la coordination du projet Sarliève dans le cadre de ces deux programmes, complétés par deux prospections thématiques du ministère de la Culture (Trément et al., 2005, 2006 et 2007).
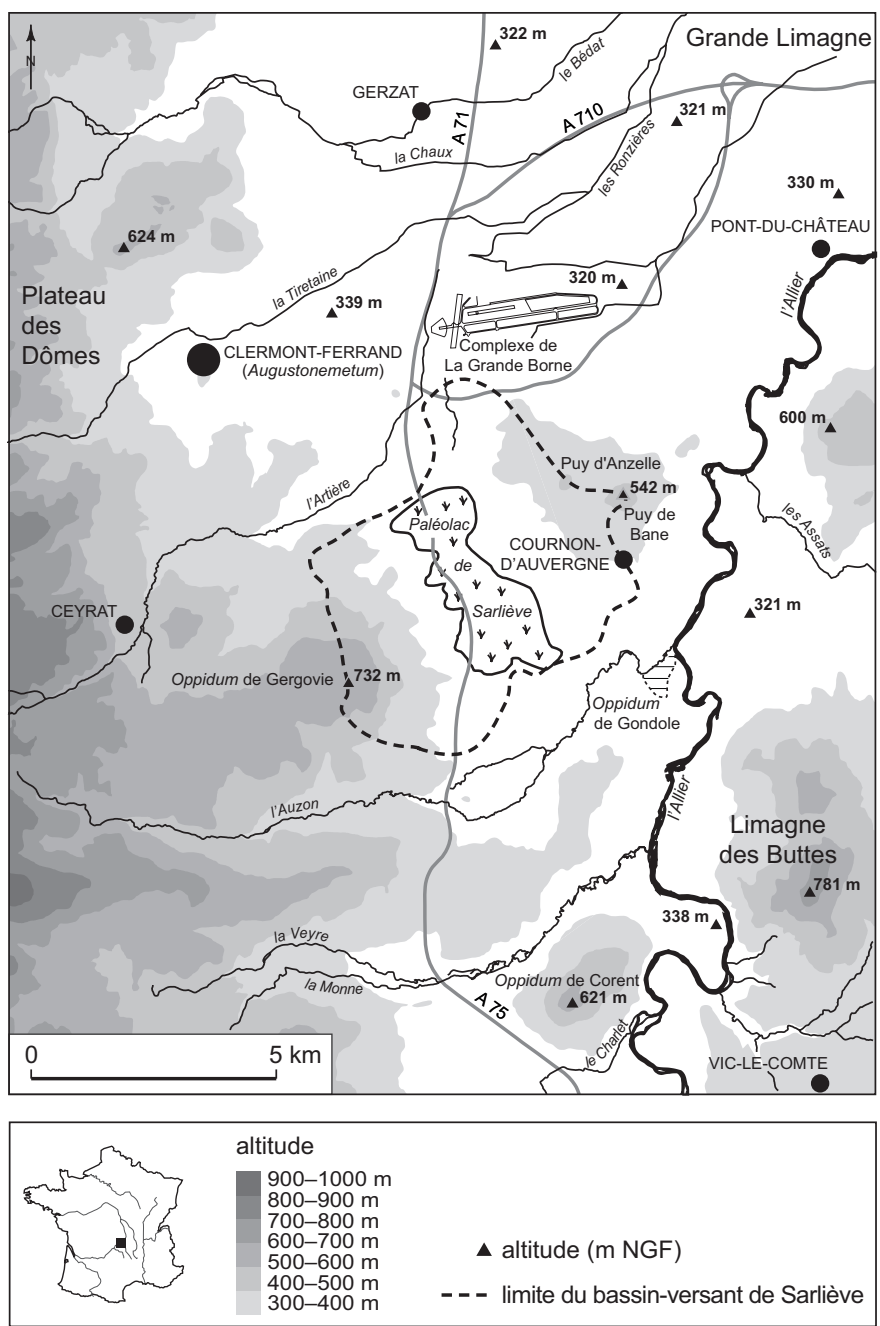

Fig. 1 - Carte de localisation du bassin de Sarliève (Puy-de-Dôme) (DAO : B. Dousteyssier et F. Trément, Université Clermont-Ferrand-II).

de 2002, dans le programme «Éclipse » du CNRS, intitulé «Interactions activités humaines, production et stockage de sédiments à l'Holocène en plaine et en moyenne montagne » ${ }^{2}$. Enfin, elles ont bénéficié des moyens et des données de l'importante opération d'archéologie préventive préalable à l'aménagement de la Grande Halle et du Zénith d'Auvergne au cœur du marais ${ }^{3}$.

F. T.

2. Responsables : J.-J. Macaire (2002-2003), puis J.-G. Bréhéret (20052006).

3. La réalisation des opérations d'archéologie préventive a été confiée à l'Association pour les fouilles archéologiques nationales (19992000), sous la responsabilité de G. Vernet (Vernet et al., 2005). 


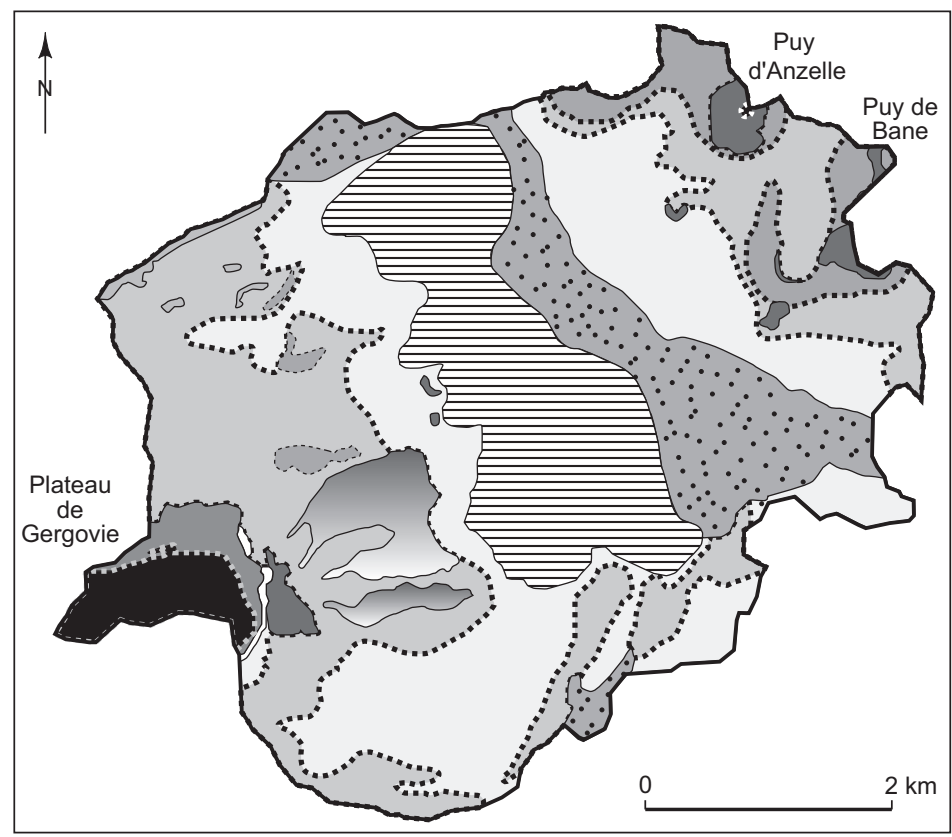

Formations superficielles

E: remplissage du lac $(K)$

$\because$ alluvions (Fv, Fx-y)

$\square$ colluvions (Cb)

$\square$ formations de versant (A)

$\square$ matériaux de déjection $(\mathrm{J})$

$\checkmark$ formations argilo-calcaires de bas de versant (CRg)

:..... matériaux fins argilo-calcaires $(\mathrm{RCg})$

Substrat

Miocène, complexe détritique argilo-quartzofeldspathique $(\mathrm{m} 1)$

Miocène, basalte (ebm)

:...... Oligocène supérieur, complexe argilo-calcaire (g3)

Oligocène supérieur, formations volcano-

sédimentaires-pépérites ( 97 )

Fig. 2 - Carte géologique du bassin-versant du marais de Sarliève

(DAO : J.-G. Bréhéret, A. Fourmont et J.-J. Macaire, d'après Jeambrun et al., 1973).

\section{PRÉSENTATION DU MILIEU}

Le marais de Sarliève se situe à quelques kilomètres au sud-est de Clermont-Ferrand, en bordure occidentale de la plaine de Limagne, au pied du plateau de Gergovie. Le bassin-versant, d'une superficie de $28,80 \mathrm{~km}^{2}$, est fermé à l'amont par des collines de marnes oligocènes et à l'aval par la terrasse fluviatile de l'Artière (Jeambrun et al., 1973) (fig. 2). L'ancien lac constitue un replat topographique d'environ $5 \mathrm{~km}^{2}$, allongé selon une direction nord-nordouest/sud-sud-est, qui s'élève à 343-344 m d'altitude (fig. 3). Il est encadré à l'est par les reliefs que constituent les puys d'Anzelle (528 m) et de Bane (542 m), et à l'ouest par le plateau de Gergovie (735 m) (fig. 4).

A. F., J.-J. M. et J.-G. B.

\section{PROBLÉMATIQUE ET MÉTHODES}

\section{PROBLÉMATIQUE GÉNÉRALE}

Trois axes de recherche ont été définis :

- la caractérisation de l'évolution du milieu dans ses différentes composantes biophysiques ; l'accent a été mis sur l'analyse des relations entre végétation, paléohydrographie et dynamiques sédimentaires dans la longue durée ;
- la caractérisation de l'histoire de l'occupation du sol et de l'exploitation du milieu par les populations successives ;

- la caractérisation de l'impact de la pression anthropique sur le milieu: modification de la couverture végétale, des dynamiques sédimentaires, pédologiques et hydrologiques.

Ces trois points de vue sur les relations sociétés-milieux sont indissociables: l'environnement est en effet conçu comme la résultante d'une interaction permanente entre composantes sociales et biogéographiques, c'est-à-dire comme un «anthroposystème ».

F. T.

\section{OBJECTIFS ET MÉTHODES}

\section{La prospection systématique du bassin}

L'enquête sur l'histoire de l'occupation du sol s'est appuyée sur un dépouillement méthodique de la bibliographie des neuf communes qui se partagent le bassin-versant et sur cinq campagnes de prospections systématiques réalisées entre 2001 et 2003 dans le cadre des stages d'archéologie de l'université Blaise-Pascal. Sur les $28,80 \mathrm{~km}^{2}$ du bassinversant, $6,30 \mathrm{~km}^{2}(22 \%)$ sont inaccessibles à la prospection : centres urbains, zones pavillonnaires et industrielles, réseau routier; $11,60 \mathrm{~km}^{2}$ ont été couverts avec une maille 


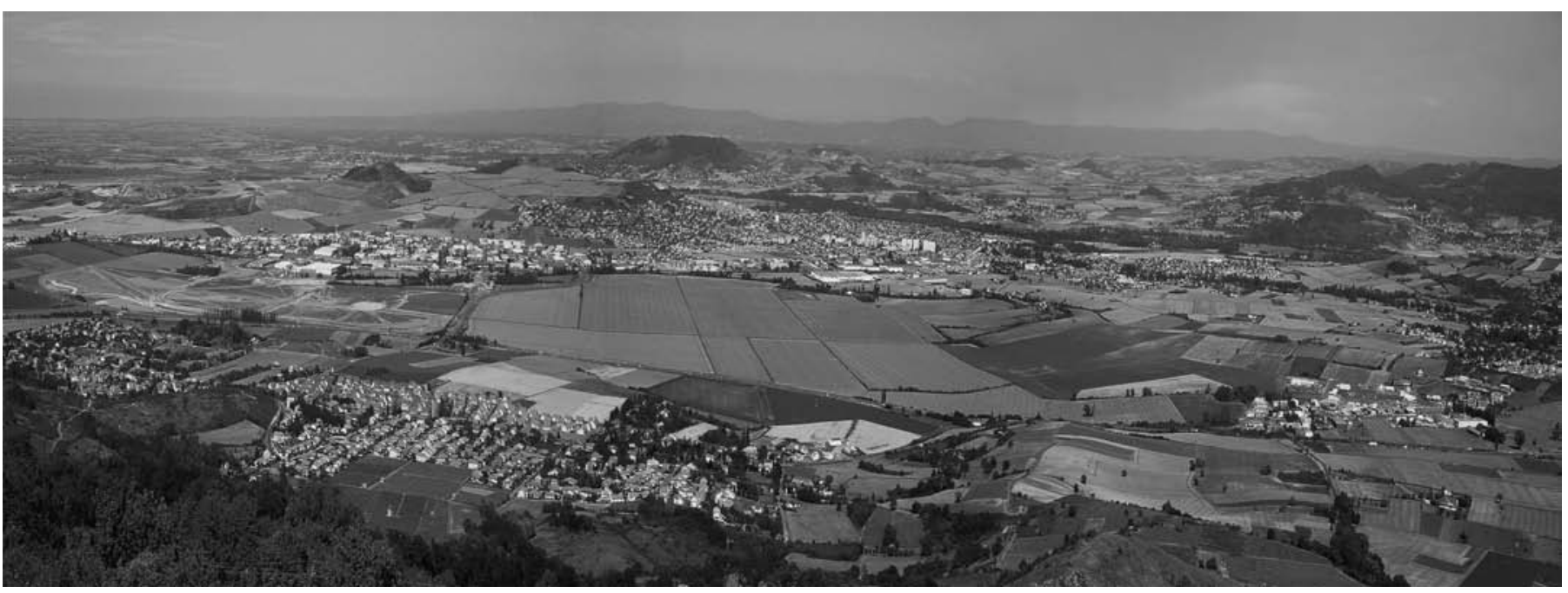

Fig. 3 - Vue panoramique du bassin de Sarliève en direction de l'est depuis le plateau de Gergovie (cliché : F. Trément, Université Clermont-Ferrand-II).

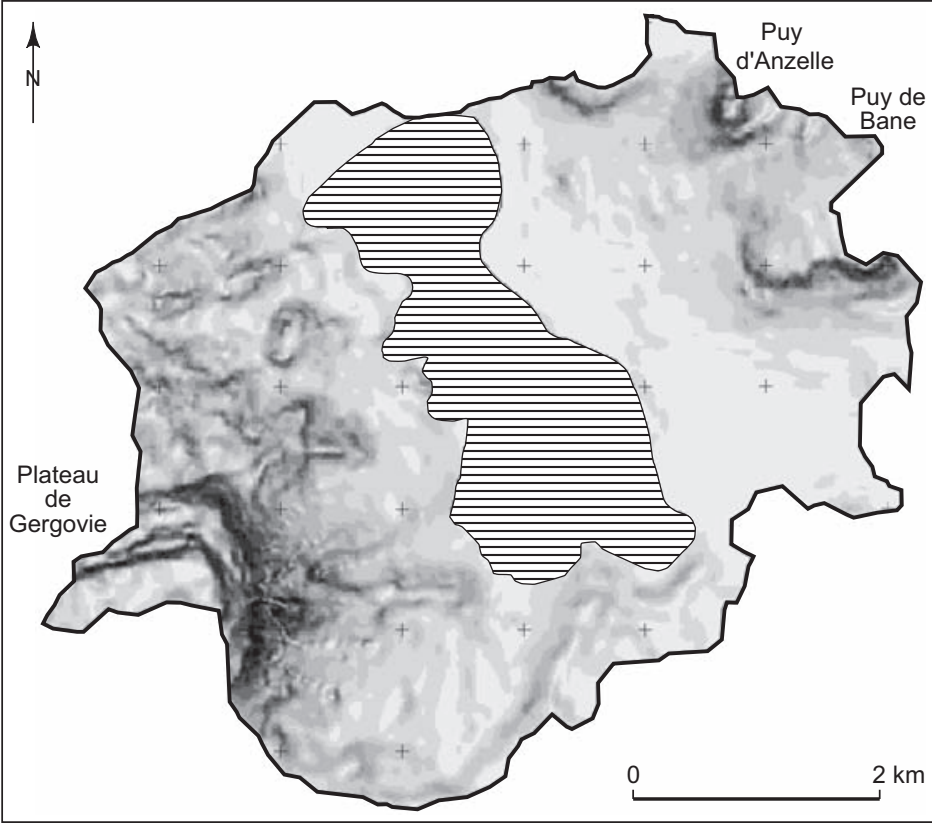

pente (en degrés)

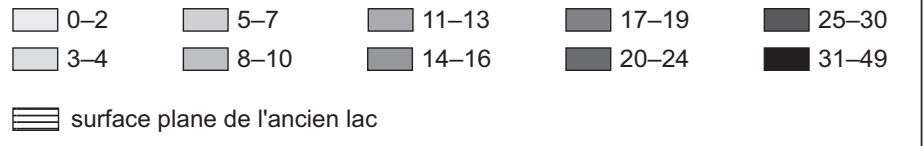

a

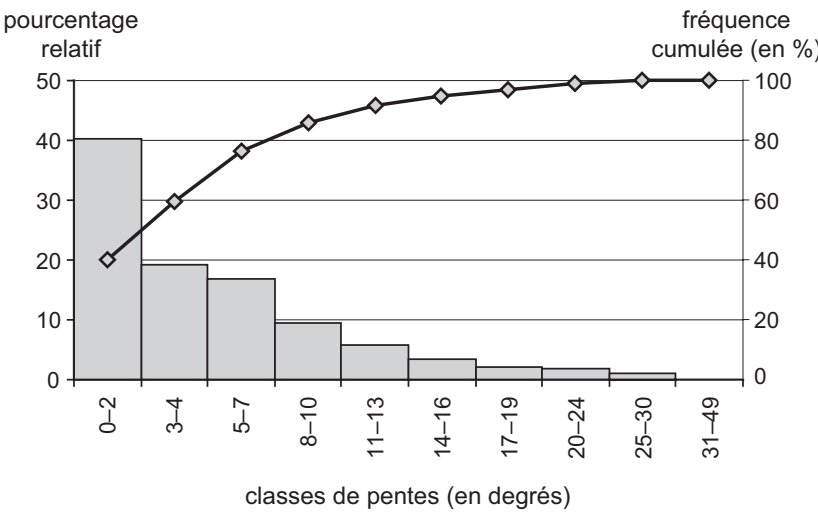

b

Fig. 4 - Les pentes du bassin-versant de Sarliève : a, carte des pentes ; b, histogramme des pentes (DAO :J.-G. Bréhéret, A. Fourmont et J.J. Macaire). 
de $10 \mathrm{~m}$, soit $51 \%$ des terrains prospectables et $40 \%$ de la superficie totale du bassin. Les zones prospectées couvrent équitablement les différentes unités physiques: plateaux, versants, vallons, basses terrasses, cuvette (fig. 5).
Le ramassage a été effectué par « unité de prospection », c'est-à-dire sur la base de la plus petite unité spatiale individualisable sur le terrain au moment de la prospection: parcelle prospectée, site, indice de site, découverte isolée ou

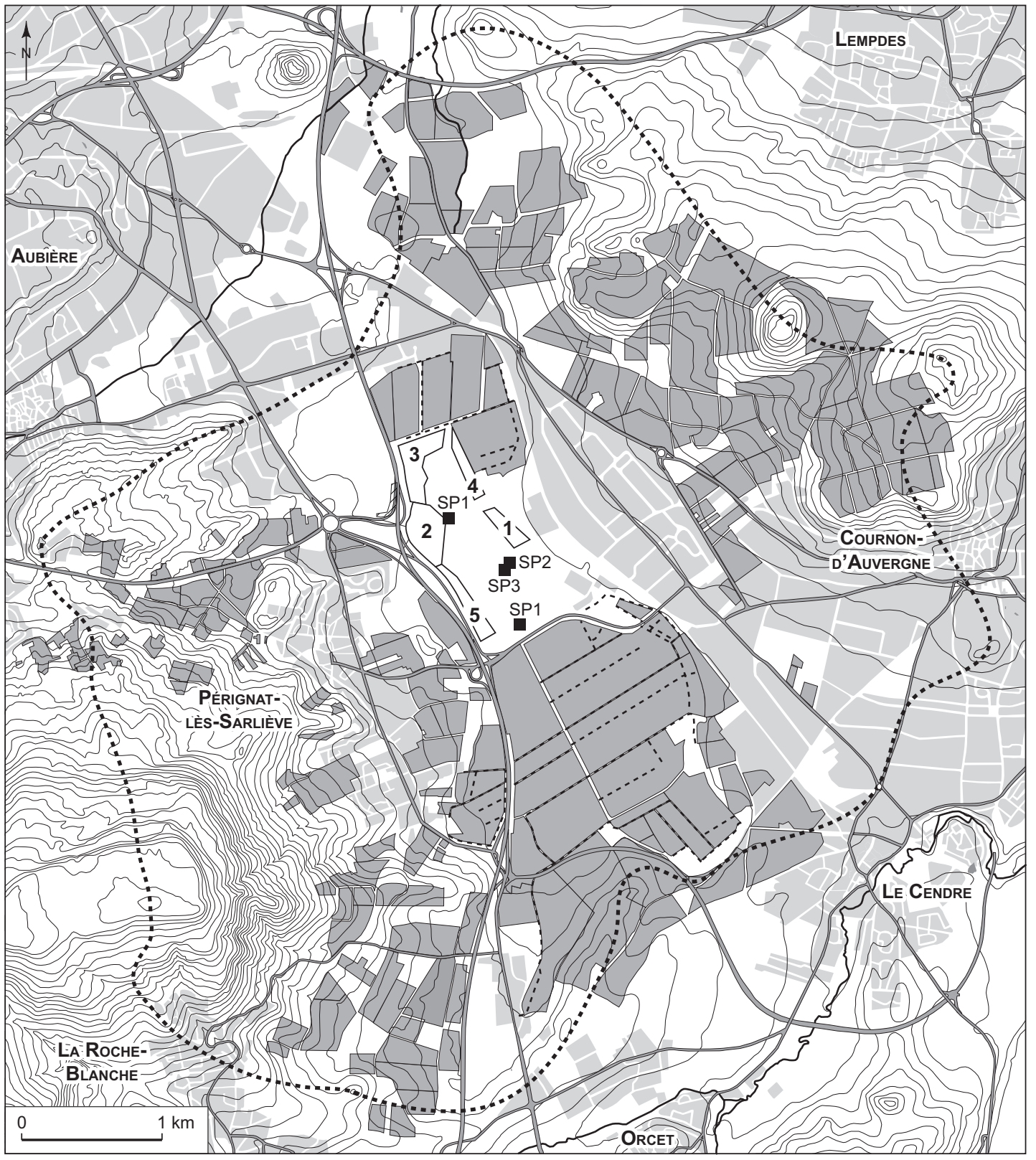

zone prospectée systématiquement

zone urbaine

.... limite du bassin-versant

1 à 5 zone d'emprise de l'opération Grande Halle avec numéro de secteur de fouille

sondage profond (SP)

Fig. 5 - Interventions archéologiques conduites dans le cadre du programme de recherche sur le bassin de Sarliève (DAO : B. Dousteyssier et F. Trément, Université Clermont-Ferrand-II). 
épandage ${ }^{4}$. Sur chaque site et indice de site, le mobilier a été ramassé de manière exhaustive.

Au total, 123 sites (dont 20 sites funéraires), 76 indices de sites et 20 découvertes isolées ont été recensés, soit 219 points correspondant à 405 occupations. Un site est défini comme une concentration de mobilier précisément délimitée dans l'espace et cohérente d'un point de vue chronologique, attestant une ou plusieurs occupations à un endroit donné. Un indice de site est défini comme un site potentiel, insuffisamment caractérisé du point de vue spatial, pour des motifs taphonomiques (érosion, recouvrement, état du couvert végétal, remaniements divers). Les découvertes isolées sont des artefacts remarquables recueillis hors de tout contexte. Enfin, les épandages, qui sont supposés résulter de pratiques agricoles anciennes (amendements), se traduisent par une présence diffuse mais récurrente de tessons de petite taille aux angles émoussés.

F. T. et B. D.

\section{Les fouilles de la Grande Halle d'Auvergne}

L'opération d'archéologie préventive de la Grande Halle d'Auvergne a été conduite sur une superficie de 90 ha préalablement à l'aménagement, par la Région Auvergne, de la partie nord de la cuvette (Vernet et al., 2005). Lors de la phase d'évaluation archéologique, la stratégie d'intervention a été la suivante :

- réalisation de quatre sondages profonds (SP1 à SP4) et d'un sondage linéaire de $50 \mathrm{~m}$ dans la zone d'étranglement du bassin; observation de la partie supérieure de la séquence lacustre sur $200 \mathrm{~m}$ de longueur sur le paléorivage oriental ; ces sondages avaient pour but de fournir des stratigraphies complètes du remplissage du bassin ;

- évaluation du potentiel archéologique par des sondages à $1 \%$ situés dans la périphérie du bassin, à l'intérieur de deux bandes, à l'est et à l'ouest, d'environ $90 \mathrm{~m}$ de large sur $1500 \mathrm{~m}$ de long (surface estimée à $270000 \mathrm{~m}^{2}$ ).

Un site archéologique important, détecté sur la bordure orientale de la cuvette, a fait l'objet d'une fouille préventive. L'intégralité de la zone archéologique estimée lors de la phase d'évaluation a été décapée, soit environ $7500 \mathrm{~m}^{2}$, tandis que le secteur situé immédiatement au sud a été

4. Les unités de prospection (UP) sont numérotées, entre crochets dans le texte, de 001 à 336 , tandis que les sites recensés dans la bibliographie sont identifiés au moyen d'un code composé des initiales de la commune et d'un numéro. Les codes utilisés pour les communes sont les suivants : CF (Clermont-Ferrand), LE (Lempdes), CO (Cournond'Auvergne), LC (Le Cendre), OR (Orcet), RB (La Roche-Blanche), PE (Pérignat-lès-Sarliève), RO (Romagnat), AU (Aubière). exploré au moyen de tranchées, ce qui a permis d'appréhender une surface complémentaire d'environ $7000 \mathrm{~m}^{2}$. À chacune de ces étapes, un protocole d'acquisition de données paléoenvironnementales a été mis en œuvre.

G. V.

\section{Les études géologiques}

Les travaux du laboratoire de Géologie des environnements aquatiques continentaux (GéEAC) ont été engagés dans le but de préciser le contexte physique de l'évolution des sociétés dans le bassin et de calculer les quantités de sédiments érodés sur les versants, déplacés et stockés dans les zones basses du bassin de la Loire au Tardiglaciaire et à l'Holocène (fig. 6). Ces objectifs impliquaient de préciser et de comparer le fonctionnement du géosystème lacustre (lac et bassin-versant) en contexte «naturel » avant anthropisation sensible, puis en contexte d'anthropisation croissante. Pour cela, les sédiments stockés dans le réceptacle lacustre ont été étudiés : faciès, compositions minéralogique et chimique, âges. Ces données permettent d'approcher les conditions de leur genèse (formation in situ ou détritisme, nature - dissoute ou solide - des apports depuis le bassin-versant, profondeur et extension de l'eau, chimie et dynamique des eaux) et les caractéristiques paléoenvironnementales influentes. Puis, l'analyse de la géométrie des dépôts lacustres a été effectuée en vue de quantifier les sédiments stockés dans le lac pour chaque phase chronologique, afin d'évaluer la quantité de matière solide ou dissoute produite par le lac ou par les versants lors de chacune d'elles. Cette approche a déjà été développée sur d'autres bassins (Gay et al., 1998 ; Bichet et al., 1999 ; Macaire et al., 1997 et 2002).

Les aspects géomorphologiques ont été traités au moyen d'un système d'information géographique (SIG). La nature et la géométrie du remplissage sédimentaire lacustre et de ses annexes (exutoire et terrasse alluviale) ont été approchées par 25 forages non destructifs réalisés avec un carottier à percussion Eijkelkamp (fig. 6), complétés par des observations dans les sondages archéologiques. Les tests de pénétrométrie statique réalisés en février 1999 par la société Fondasol-Auvergne au moyen d'un pénétromètre de $100 \mathrm{KN}$ ont été utilisés pour préciser la géométrie du remplissage (fig. 7). La lithologie du bassin-versant (roches et sols) a été étudiée par quatre forages carottés, de nombreux forages à la tarière manuelle et l'observation de coupes.

Les composantes minérales ont été analysées : teneur en carbonates par calcimétrie, composition par diffraction 


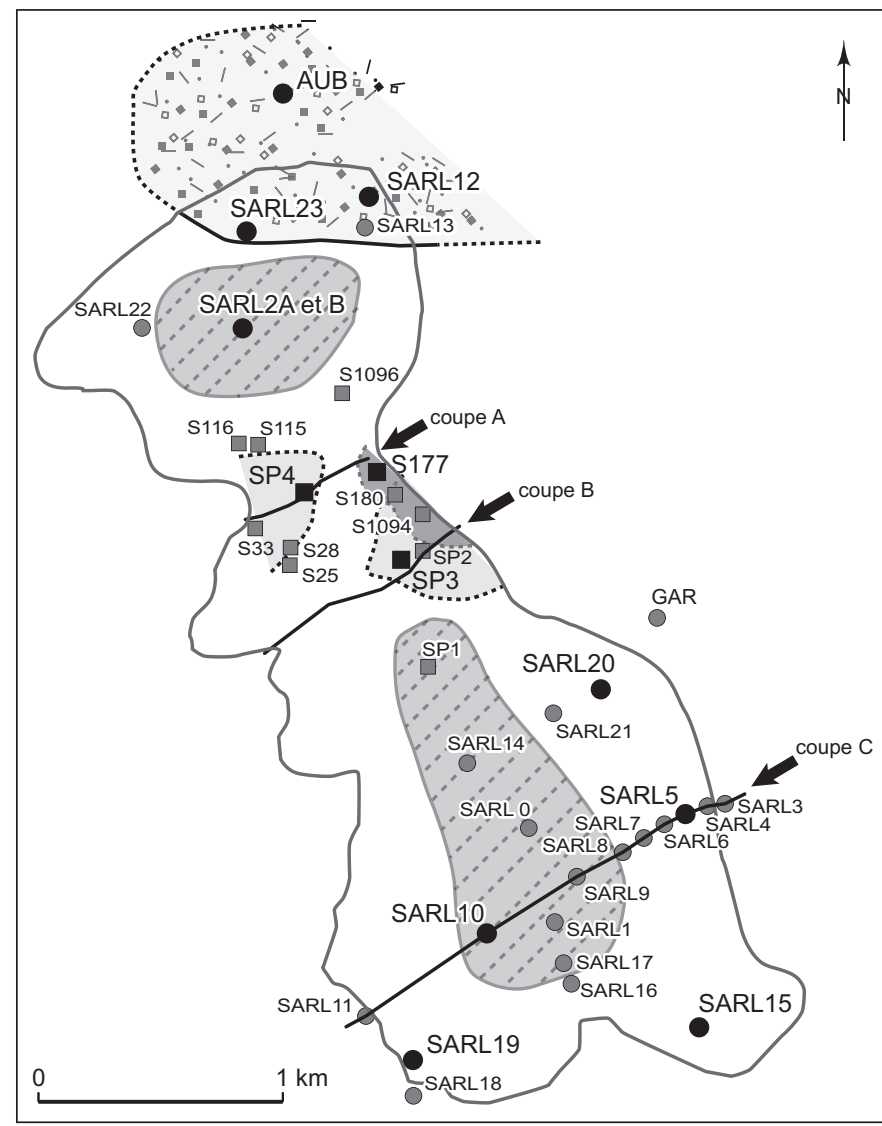

forage carotté décrit fosse, sondage ou sondage profond décrit forage carotté non décrit $\square$ fosse, sondage ou sondage profond non décrit $\therefore \because$ zone de bassin

0. zone à «coulées boueuses» ........ zone de delta

1...2. ( 1 : delta ancien, 2 : delta récent)

contour du marais

Fig. 6 - Localisation des prélèvements des principales zones de sédimentation et des forages ou sondages effectués dans le bassin de Sarliève : $S$, sondage; SP, sondage profond; SARL, carottage; GAR, gare de Cournon (DAO : J.-G. Bréhéret, A. Fourmont et J.-J. Macaire).

des rayons X (DRX) avec un diffractomètre Rigaku à anticathode $\mathrm{Cu}$. Une évaluation semi-quantitative de chaque minéral a été faite en pourcentage par mesure de l'aire du pic de plus forte intensité du minéral rapportée au total des aires des pics les plus intenses des minéraux présents. La minéralogie des carbonates a été précisée grâce à l'utilisation d'un étalon interne de silicium et par des observations en microscopie électronique à balayage (MEB). Les composantes organiques ont fait l'objet d'analyses par pyrolyse Rock-Eval 6 et d'un examen des palynofaciès en microscopie optique.

A. F., J.-J. M. et J.-G. B.

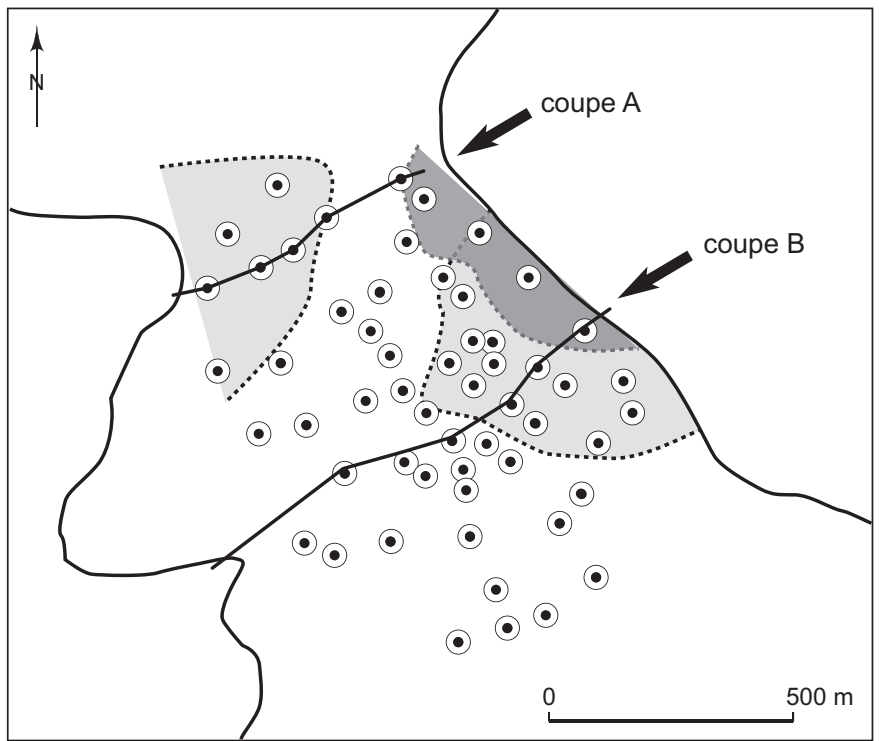

- test de pénétrométrie statique

trait de coupe 12 zone de delta (1: delta ancien, 2 : delta récent)

Fig. 7 - Localisation des tests de pénétrométrie statique réalisés dans la zone de delta du bassin de Sarliève (source : Fondasol-Auvergne ; DAO : J.-G. Bréhéret, A. Fourmont et J.-J. Macaire).

\section{Palynologie et microfossiles non polliniques}

Un carottage (SARL 2B) réalisé à la sonde russe dans la partie nord du bassin et des prélèvements tous les $3 \mathrm{~cm}$ sur la coupe du sondage profond SP4 ont permis d'obtenir une série continue de résultats polliniques, du milieu de l'Atlantique à la période subactuelle. Des prélèvements effectués sur des puits et fossés protohistoriques viennent compléter ces données.

L'étude des microfossiles non polliniques (spores d'algues, de champignons, de mousses, des cyanobactéries, des kystes d'origine variée), abondants dans ces sédiments lacustres, a enrichi les données paléoenvironnementales du diagramme pollinique (López Sáez et al., 1998). Elle a permis en outre d'apprécier les tendances de variation du niveau de l'ancien lac.

Tous les échantillons ont été traités suivant la méthode classique de concentration en liqueur dense (Thoulet $\mathrm{d}=2$ ) après décarbonatation et désilicification, puis traitement à la potasse (Argant, 1990). Les culots obtenus pour le carottage SARL 2B ont été utilisés à la fois pour l'analyse pollinique et pour celle des microfossiles non polliniques.

J. A., B. P. et J. A. L. S. 


\section{Carpologie}

L'analyse carpologique d'une structure fossoyée et de quatre puits exhumés dans la fouille de la Grande Halle a permis d'appréhender le milieu et son exploitation par l'homme. La carpologie donne un inventaire des plantes domestiques, des plantes sauvages et des mauvaises herbes des cultures. Ces espèces sont réparties dans le paysage en fonction de leurs affinités écologiques, nommées unités phytosociologiques ou groupements. Les groupements d'adventices ou mauvaises herbes sont associés aux cultures céréalières (moissons) ou aux cultures sarclées (Bournérias et al., 2001). Des analyses statistiques multivariées de type AFC (analyses factorielles des correspondances) ont permis d'identifier des remaniements dus aux pratiques agricoles et leur évolution entre le second âge du Fer et l'époque romaine.

Le choix s'est porté sur dix unités stratigraphiques qui, à la fouille, paraissaient les plus prometteuses pour fournir des données paléoenvironnementales. Datés par C. MennessierJouannet grâce aux céramiques, ces ensembles s'échelonnent entre La Tène B2/C1 (300-250 av. J.-C.) et les débuts de la période augustéenne. Les prélèvements ont livré un total de 10412 restes (semences carbonisées entières et fragmentées). Compte tenu de l'état de conservation des vestiges et de la nature des sédiments, la technique de récupération utilisée a été la flottation simple, pratiquée sur une colonne de tamis à maille de $2 \mathrm{~mm}$ et $0,5 \mathrm{~mm}$. Le tri et la détermination ont été réalisés sous loupe binoculaire. L'identification a été effectuée à l'aide d'atlas de références et de la collection de semences actuelles et fossiles du Centre d'anthropologie de Toulouse.

M. C. et P. M.

\section{UN LAC AU MOYEN ÂGE}

L'étude du lac de Sarliève au Moyen Âge repose sur la synthèse d'une documentation morcelée géographiquement et discontinue dans le temps. Les documents écrits sont rares avant le $\mathrm{XIII}^{\mathrm{e}} \mathrm{s}$ : : ils se réduisent à quelques mentions dans le cartulaire de Sauxillanges. Ils se multiplient à partir du XIII ${ }^{\mathrm{e}} \mathrm{s}$. mais, du fait de leurs origines diverses, ils ne donnent que des visions partielles et fragmentaires, qui rendent difficile toute synthèse. Les principaux proviennent du fonds des Prémontrés de Saint-André à Clermont-Ferrand, des archives relatives à l'administration de la Terre royale d'Auvergne, du fonds du chapitre de la Sainte-Chapelle de Riom.
Les plus anciens témoignages écrits sur le lac de Sarliève datent des environs de l'an mil, sous forme de simples mentions dans des confins, qui attestent son existence, sans rien nous apprendre sur son régime. Le partage du lac entre sept communes, autrefois paroisses, témoigne de la valeur attachée par les populations voisines aux ressources de ce terroir jadis mi-lacustre, mi-marécageux, et suppose une histoire complexe (fig. 8). Les eaux et les marais de la cuvette de Sarliève fournissaient aux riverains des ressources originales, auxquelles correspondaient des paysages originaux. Des textes du XI ${ }^{\mathrm{e}}$ s. relatifs au lac voisin de la Narce (commune de La Sauvetat, immédiatement au sud d'Orcet) donnent une idée des ressources et des modes d'exploitation des eaux et des marais, qui fournissaient des poissons, ainsi que des roseaux pour les toitures et le chauffage, avec également des possibilités de défrichements. Le rapprochement avec le lac de Sarliève est d'autant plus légitime qu'un texte contemporain désigne la Narce par un diminutif évoquant un petit lac par opposition au grand lac de Sarliève.

Une partie de la cuvette de Sarliève était en eau libre (des textes parlent de l'«eau claire») et la pêche y était active : une pêcherie et une rente en poissons d'un demipied de long sont mentionnées en 1224. Aux XIV et XVI ${ }^{\mathrm{e}}$ s., des baux et des procès révèlent l'importance attachée à la pêche par les seigneurs et par les habitants : ils décrivent des aménagements et des engins destinés à prendre les poissons, filets et nasses (« pantennes », « trubles », « nassons ») ; une livraison d'anguilles figure dans un bail en 1364 (c'est le seul poisson attesté). L'installation d'engins de pêche dormants suppose une profondeur d'eau suffisante qui rendait nécessaire l'utilisation d'embarcations. Les pêcheurs, habitants des villages voisins, prenaient à ferme des lots de pêche et construisaient sur les rives des cabanes pour s'abriter et ranger leur matériel.

Une autre partie de la cuvette était marécageuse et les plantes de marais représentaient une ressource pour les riverains: au XIII ${ }^{\mathrm{e}}$ s., les Prémontrés de Saint-André acquirent des roselières dans le sud-ouest du lac et en louèrent de nouvelles au XIV $\mathrm{e}$. au nord-ouest, dans la seigneurie d'Aubière. Les textes font état d'une végétation de terrain humide (roselières, cannes, mottes, roques), sans qu'il soit possible d'identifier les espèces botaniques mentionnées. Mais il est certain que cette végétation représentait une ressource appréciée et recherchée par les riverains. Ces terrains marécageux étaient traversés par des sentiers tracés par les passages répétés des pêcheurs et autres usagers.

Une partie des rives marécageuses de la cuvette était consacrée aux herbages et utilisée comme pacages, sous 


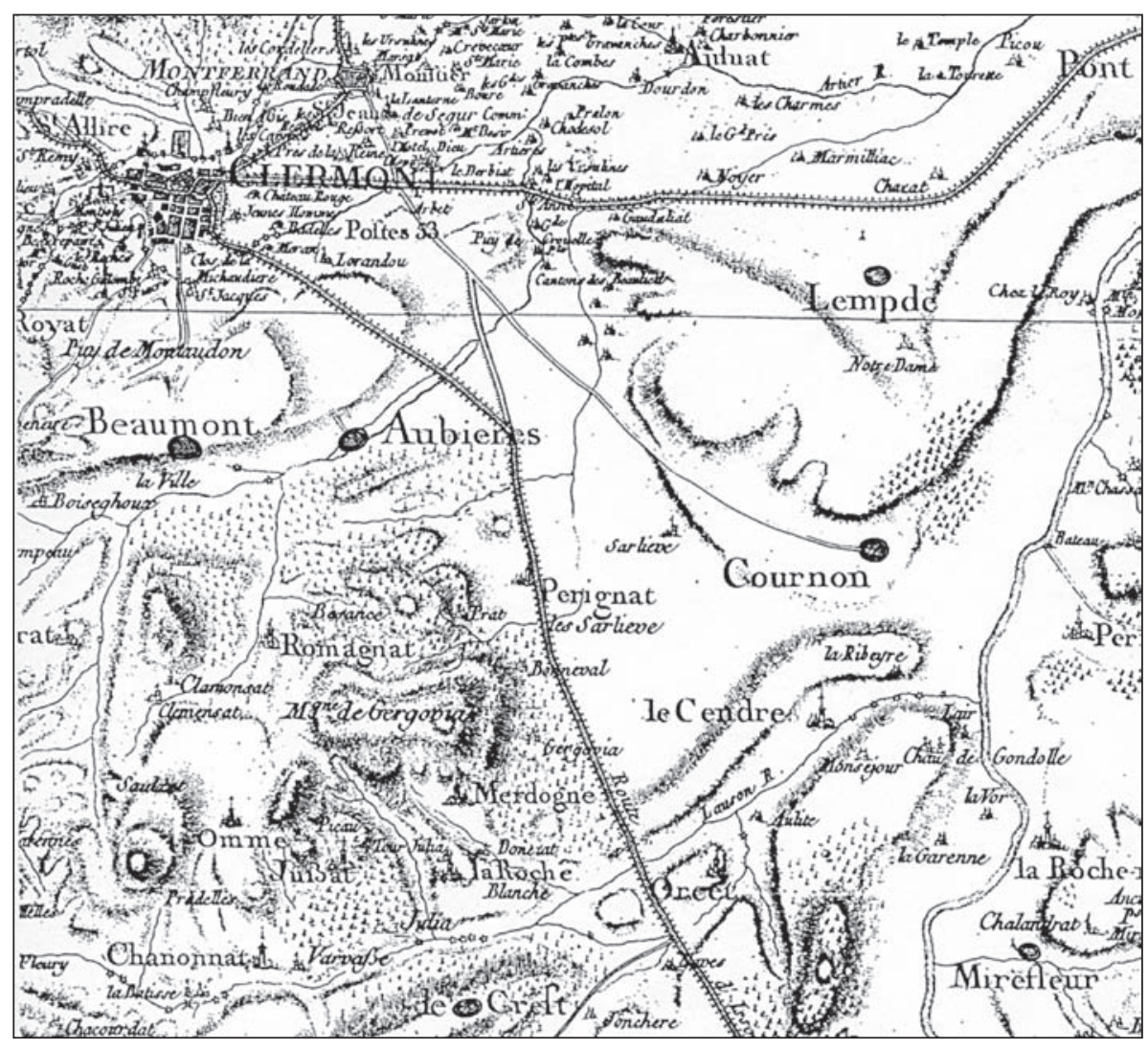

Fig. 8 - Extrait de la carte de Cassini montrant le lac asséché de Sarliève (Archives nationales).

forme soit de droits d'usage, soit de communaux au profit des communautés seigneuriales et villageoises limitrophes; celles-ci étaient très attachées à ces pratiques qui fournissaient des ressources complémentaires non négligeables pour l'élevage du bétail. Tout donne à penser que de nouveaux espaces furent ainsi conquis sur les marais, voire gagnés à la culture.

Les domaines créés par des religieux sur les rives du lac, souvent sur les marges des paroisses et des seigneuries, incorporèrent dans leur territoire des secteurs de marais et de lac. Les domaines de ce type les mieux connus sont celui de Sarliève/Fontentige, sur la rive septentrionale, aux mains des Prémontrés de Saint-André de Clermont depuis la fin du XII ${ }^{\mathrm{e}}$ s., et celui de Bonneval, sur la rive occidentale, aux mains des Cisterciens de Bonnaigue, attesté depuis le milieu du $\mathrm{XIII}^{\mathrm{e}} \mathrm{s}$. Les deux domaines possédaient des roselières et des pêcheries; depuis le XIII ${ }^{\mathrm{e}}$ s., le secteur sudouest du lac était indivis entre les deux établissements. Le domaine et prieuré de Prat, dépendant des Cisterciennes de L'Éclache, bien attesté à partir des XIII ${ }^{\mathrm{e}}$-XIV ${ }^{\mathrm{e}}$ s., exerçait également des droits sur le lac.
À partir de la fin du XVI ${ }^{\mathrm{e}}$ s., dans le cadre d'une nouvelle politique royale en matière de marais, l'idée s'imposa de remplacer l'exploitation du lac sous sa forme traditionnelle, morcelée entre les seigneuries riveraines, par une mise en valeur globale: des promoteurs étrangers à la province (les Bradley, puis les Strada) procédèrent, non sans mal en raison de la résistance des usagers traditionnels, à des remembrements et à des travaux d'assèchement, créant un nouveau domaine sur la rive orientale (fig. 9 et 10).

G. F.

\section{L'OCCUPATION DU SOL DANS LE BASSIN DE SARLIÈVE DU NÉOLITHIQUE AU MOYEN ÂGE}

Les prospections systématiques, complétées par les données des fouilles et les découvertes anciennes, permettent de restituer l'évolution de l'habitat avec une précision remarquable. Ce qui frappe, à la lecture des cartes archéologiques, c'est, d'une part, la densité remarquable des sites de toutes périodes, et, d'autre part, les contrastes très marqués dans les dynamiques de l'occupation du sol (fig. 11). 


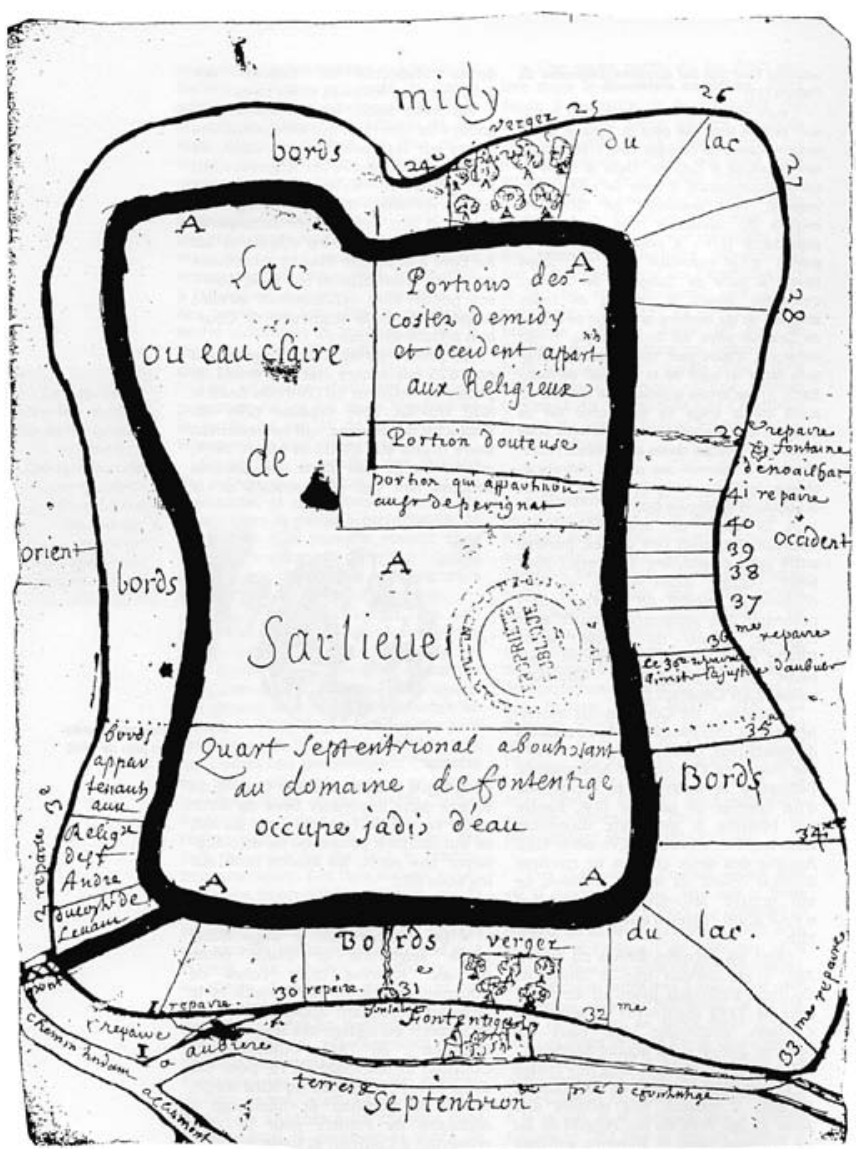

Fig. 9 - Plan de la plaine de Sarliève vers 1663 (Archives départementales du Puy-de-Dôme, $n^{o}$ inv. 16H82c.2).

\section{LE NÉOLITHIQUE}

\section{LE NÉOLITHIQUE ANCIEN}

Pas moins de 21 sites, 7 indices de sites et 12 découvertes isolées sont attestés pour le Néolithique. Le Néolithique cardial récent ou Épicardial (fin du Néolithique ancien) est représenté par deux tessons de céramique à pastillage recueillis, l'un sur un site de bas de versant occupé également au Néolithique moyen et final [264, fig. 12], l'autre sur un site du Bronze ancien et moyen [274, fig. 13] localisé au sommet du plateau séparant les puys d'Anzelle et de Bane. Bien que quantitativement négligeables, ces deux découvertes sont suffisamment exceptionnelles pour être soulignées. On connaît en effet très peu de sites de cette période en Auvergne. Si l'on excepte quelques découvertes dans le sud du Velay, les très rares sites ou indices déjà connus pour cette période dans la région se localisent tous immédiatement au nord du bassin de Sarliève : Pontcharaud 2 et son extension du Brézet-Est (Clermont-Ferrand), la rue des Quatre-Passeports (Clermont-Ferrand), le parc logistique de Gerzat. En périphérie de cet ensemble s'ajoutent les sépultures individuelles en fosse de la Madeleine à Pont-du-Château (5580-5430 av. J.-C.) et du Creux-Rouge à Clermont-Ferrand (5200-4720 av. J.-C.). Il semble donc bien, en l'état des connaissances, que le bassin de Clermont-Ferrand et plus particulièrement cette zone de contact entre Limagne des Marais et Limagne des Buttes, aient constitué l'un des terroirs privilégiés de néolithisation dans le Massif central.

\section{LE NÉOLITHIQUE MOYEN}

Le Néolithique moyen chasséen est particulièrement bien documenté, avec 17 sites, 3 indices de sites et 5 découvertes isolées (fig. 12). Les implantations se localisent très préférentiellement sur les versants et les piémonts du plateau de Gergovie à l'ouest et des puys d'Anzelle et de Bane à l'est. Le sommet du plateau de Gergovie est également occupé. Mais les zones basses ne paraissent pas désertées pour autant, comme en témoigne la présence de quelques établissements sur la terrasse orientale et à l'extrémité nord du bassin, à son débouché. L'altitude moyenne des implantations est de 412 m NGF. L'examen de leur situation topographique révèle que les occupants ont préféré systématiquement les replats et les terrains les moins pentus, quelle que soit leur orientation.

\section{LE NÉOLITHIQUE RÉGENT/FINAL}

L'occupation du bassin de Sarliève au cours du Néolithique récent/final est encore très mal connue. Cette phase chronoculturelle est en effet difficile à reconnaître clairement sur la base des trouvailles de surface, en partie en raison d'un problème de caractérisation des productions céramiques ; et si les caractères des industries sur silex sont plus évidents (armatures évoluées, fragments de poignard, grands racloirs), de tels ramassages sont trop rares pour être significatifs. Il n'en demeure pas moins évident que cette période marque une nette rupture avec le Néolithique moyen chasséen: rupture du mouvement extensif vers les surfaces agricoles, abandon des sites, phénomène de repli, développement d'une économie probablement plus orientée vers le pastoralisme. Les implantations semblent s'orienter sur l'arrière-pays, sur les coteaux et les reliefs périphériques, qui sont également le siège de sépultures collectives en fosse (plateau de Corent) ou en mégalithes (la Grotta à Cournols). 


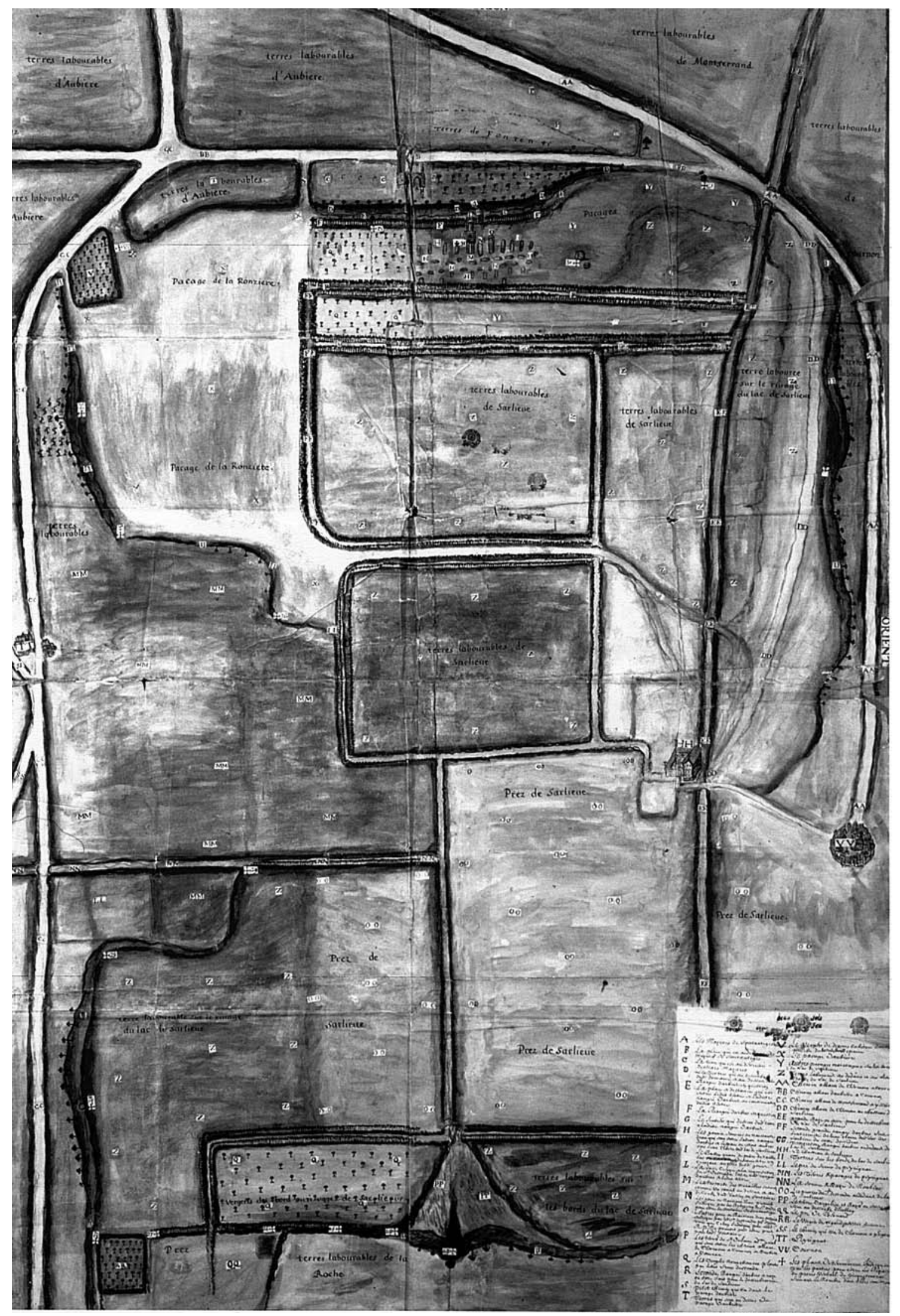

Fig. 10 - Plan de la plaine de Sarliève dressé en 1678 par le peintre clermontois Guillaume Duclos, sur lequel on peut situer la grande rase creusée pour le dessèchement du lac (Archives départementales du Puy-de-Dôme, meuble à plans noo 1152). 


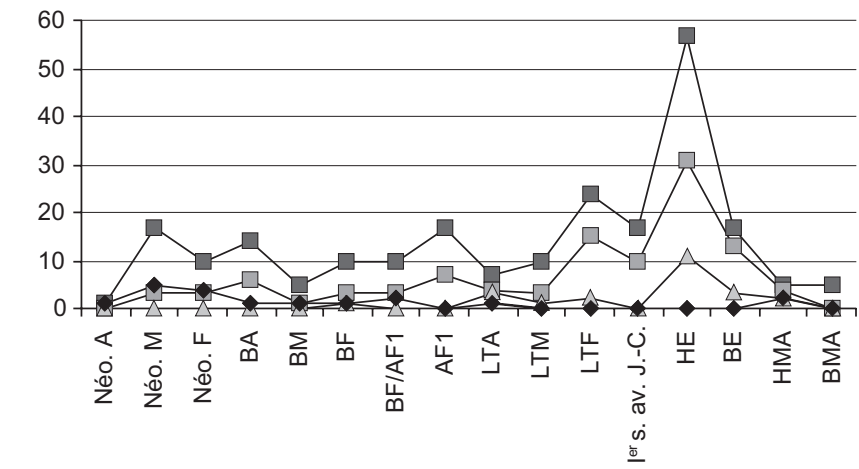

$\neg-$ site $\longrightarrow$ indice $\longrightarrow$ funéraire $\longrightarrow$ découverte isolée

1

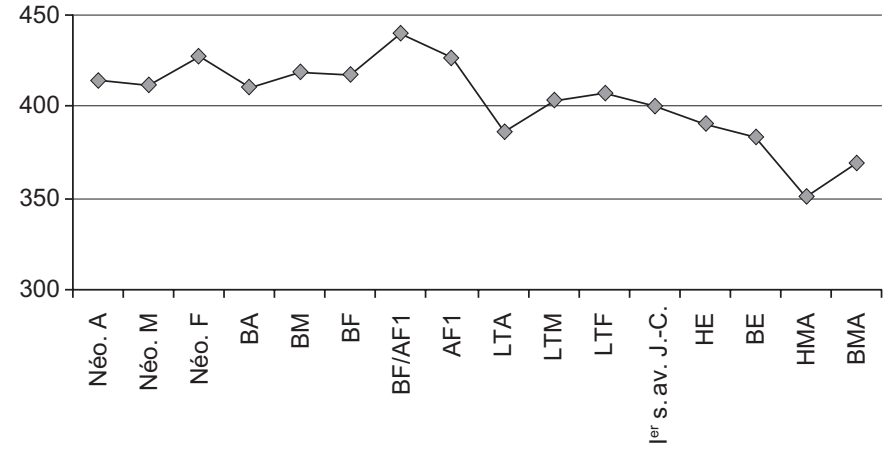

$\sim$ site

2

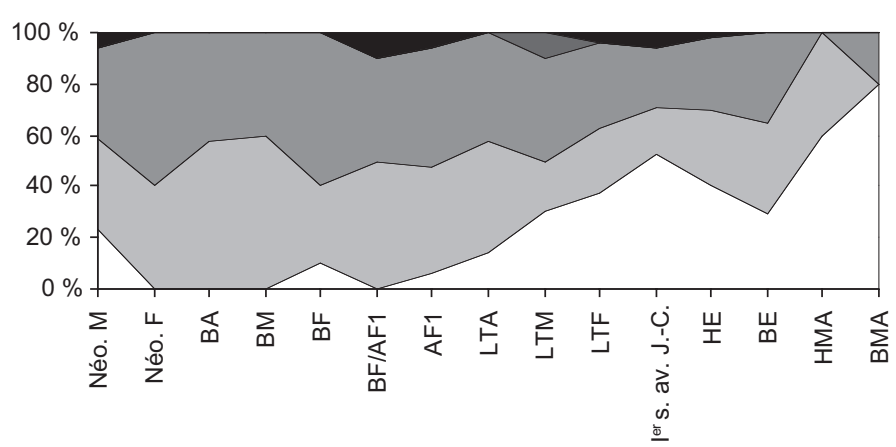

$A>700 \square 500<A<700 \square 400<A<500 \square 350<A<400 \square A<350$
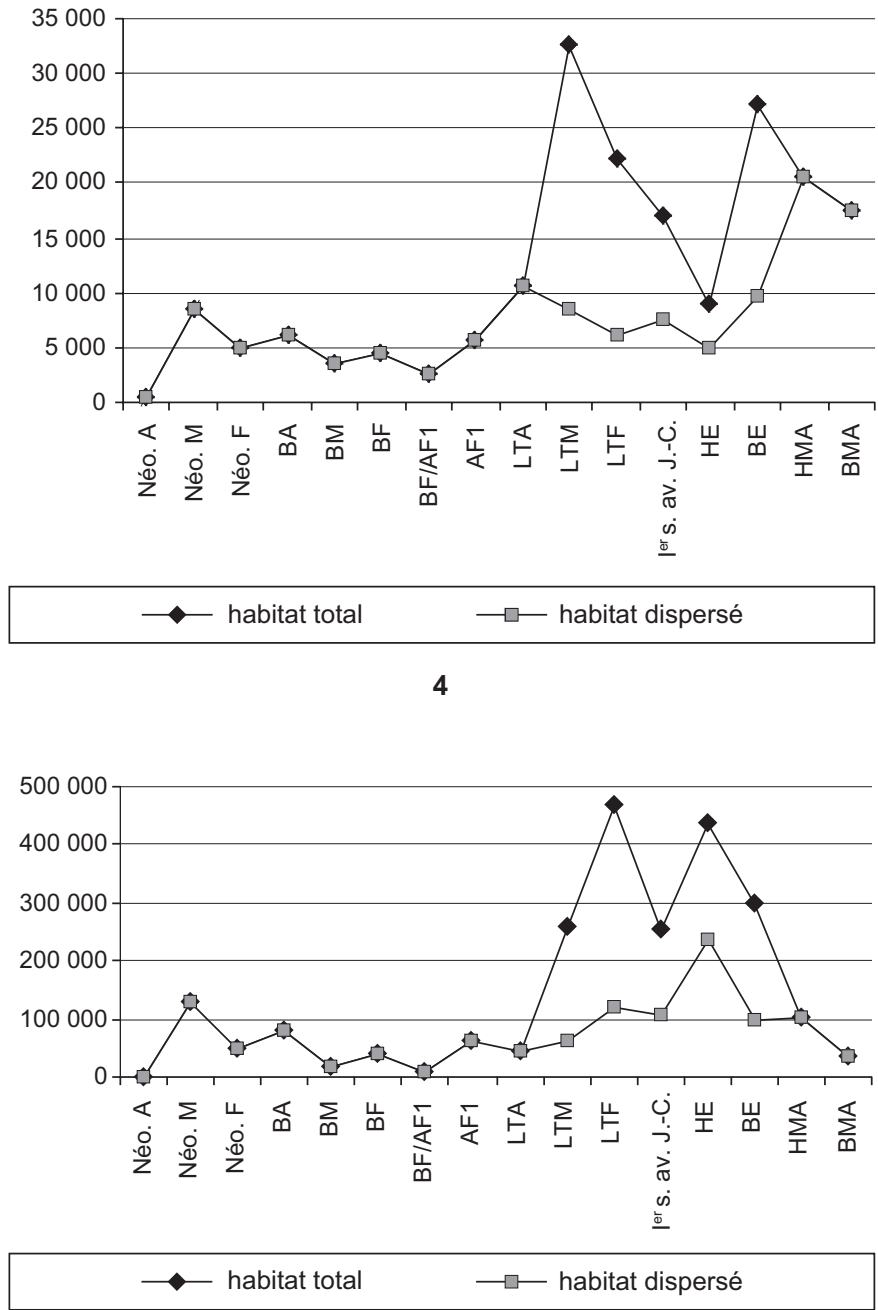

5

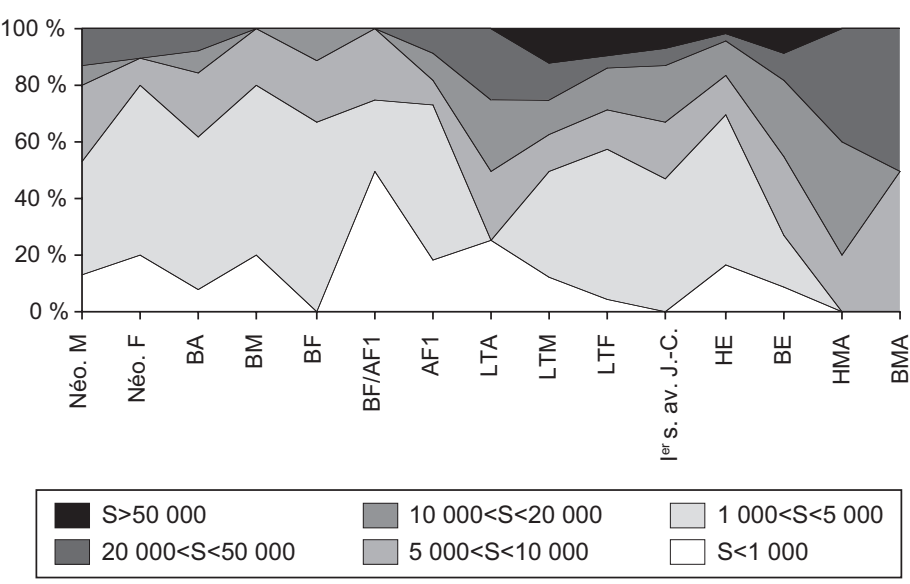

Fig. 11 - Dynamique de l'occupation du bassin de Sarliève: 1, quantification des sites, indices de sites, sites funéraires et découvertes isolées; 2, altitude moyenne des sites (en $m N G F)$; 3, répartition des sites par classes d'altitude (en $m N G F)$; 4, superficie moyenne des sites (en $\left.m^{2}\right)$; 5 , superficie cumulée des sites (en $m^{2}$ ); 6, répartition des sites par classes de superficie (en $\left.\mathrm{m}^{2}\right)$; Néo. A., Néolithique ancien ; Néo. M., Néolithique moyen ; Néo. F., Néolithique final ; BA, Bronze ancien ; BM, Bronze moyen; BF, Bronze final; BF/AF, transition Bronze final/Âge du Fer ; LTA, La Tène ancienne; LTM, La Tène moyenne; LTF, La Tène finale ; HE, Haut-Empire; BE, Bas-Empire ; HMA, haut Moyen Âge ; BMA, bas Moyen Âge (DAO : F. Trément, Université Clermont-Ferrand-II). 


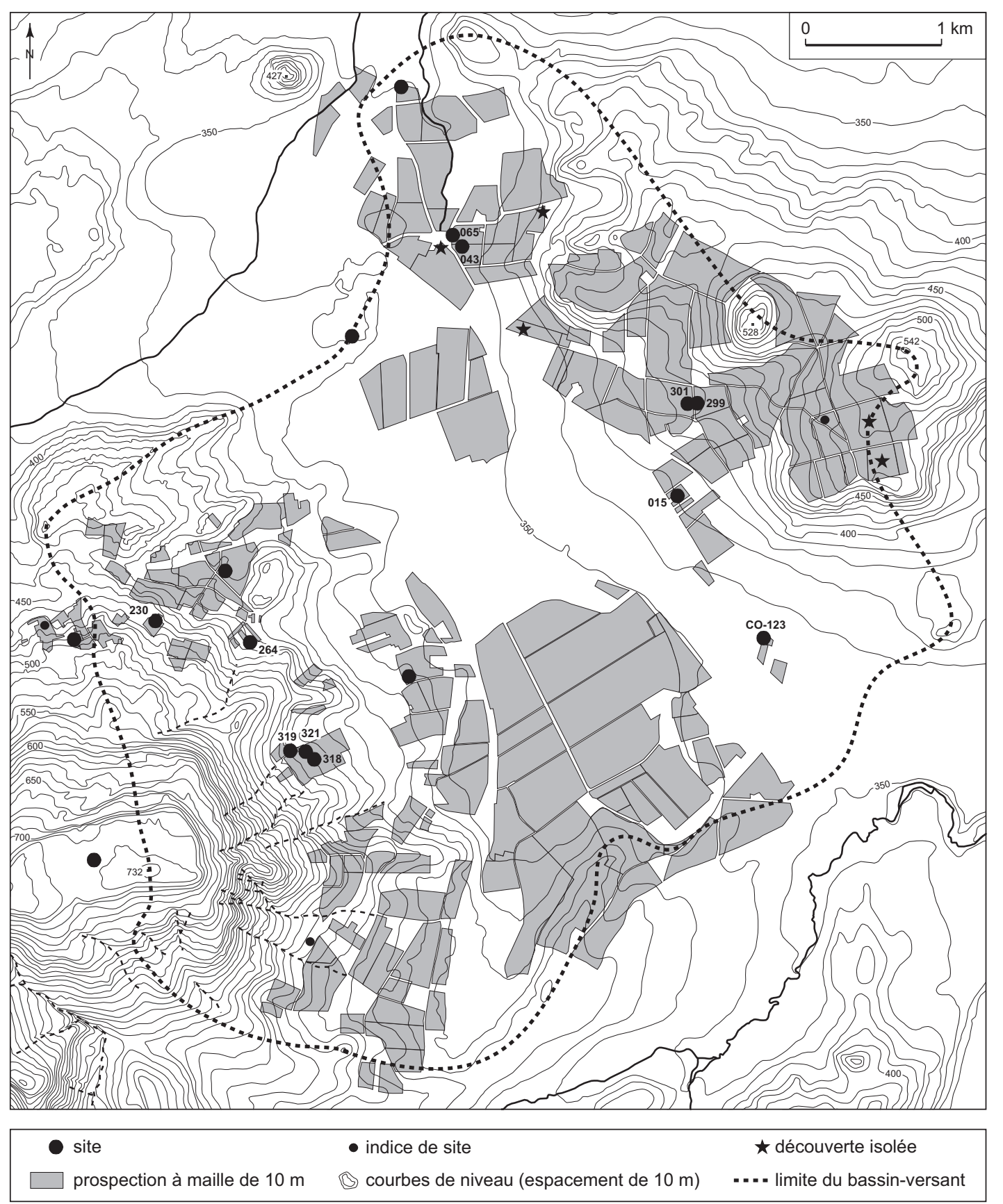

Fig. 12 - L'occupation du bassin de Sarliève au Néolithique moyen (DAO : B. Dousteyssier et F. Trément, Université Clermont-Ferrand-II).

\section{L'ÂGE DU BRONZE}

\section{LE BRONZE ANCIEN}

Le Bronze ancien est particulièrement bien documenté, avec 14 sites, 6 indices et 1 découverte isolée (fig. 13).
Comme au Néolithique moyen, l'essentiel des implantations se regroupe sur les replats des versants de Gergovie, sur les pentes du puy d'Anzelle et sur les piémonts. Deux indices de sites localisés au débouché du bassin vers le nord suggèrent une occupation de ce secteur, déjà fréquenté 


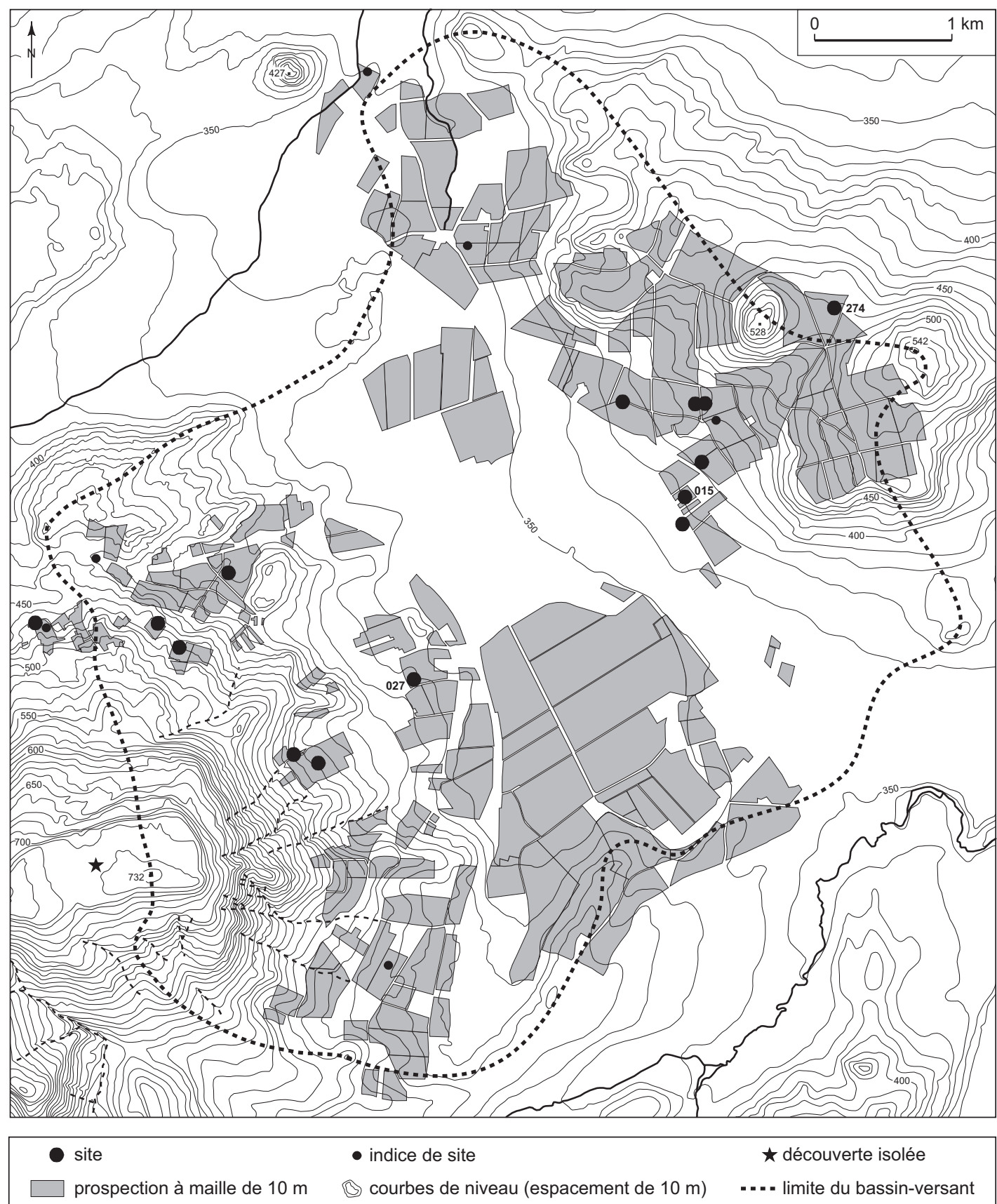

Fig. 13 - L'occupation du bassin de Sarliève au Bronze ancien (DAO : B. Dousteyssier et F. Trément, Université Clermont-Ferrand-II).

au Néolithique moyen. Le fond du vallon de Maréchal est également occupé par un établissement. En revanche, l'habitat paraît déserter les terres les plus basses, en périphérie de la cuvette de Sarliève. De fait, aucun site n'est implanté à moins de 360 m NGF. C'est là peut-être la conséquence du haut niveau relatif du plan d'eau attesté au Subboréal.

Les prospections semblent donc bien confirmer les observations de G. Loison (2003, p. 9 et 118) relatives à la concentration exceptionnelle des gisements de plein air du 


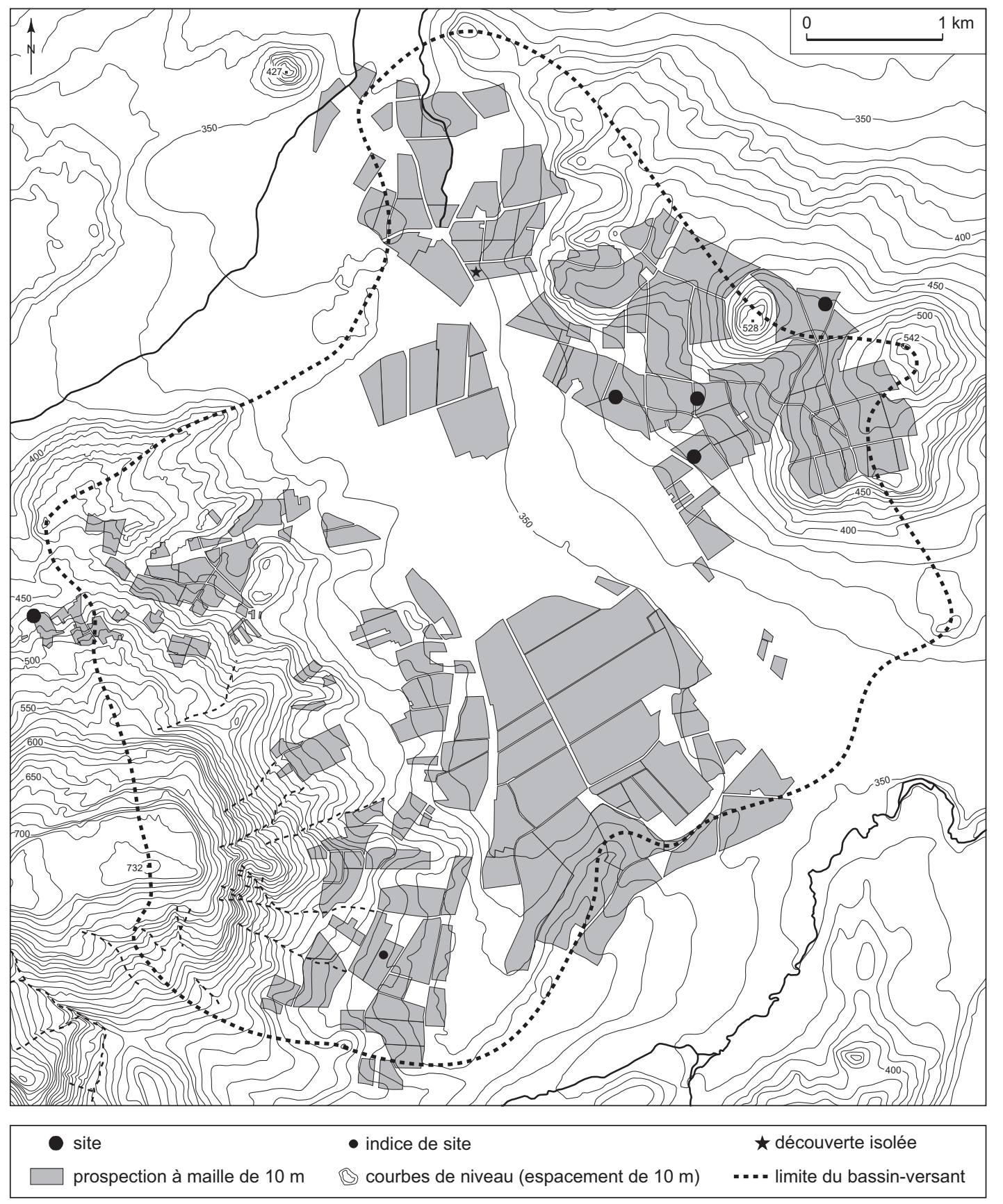

Fig. 14 - L'occupation du bassin de Sarliève au Bronze moyen (DAO : B. Dousteyssier et F. Trément, Université Clermont-Ferrand-II).

Bronze ancien sur les marges méridionales de la Grande Limagne. Pas moins de six établissements de cette période ont été fouillés ou observés à l'occasion de travaux dans le bassin de Sarliève et en périphérie immédiate, dont quatre implantés sur les pentes du plateau de Gergovie. Une telle densité d'occupation pourrait s'expliquer, selon G. Loison (ibid., p. 129), par un «développement des techniques agropastorales » autorisant le dégagement des surplus de production nécessaires à l'acquisition des objets de prestige exhumés, notamment en contexte funéraire. 


\section{LE BRONZE MOYEN}

Le Bronze moyen n'est attesté que par 5 sites, 1 indice de site et 1 découverte isolée (fig. 14). Tous présentent des signes d'occupation au Bronze ancien. Il est malaisé d'interpréter la diminution apparente du nombre de sites. La localisation des implantations suggère que, plus que jamais, les zones basses et les piémonts sont désertés. En effet, aucun site n'est attesté en dessous de $370 \mathrm{~m}$ d'altitude. $60 \%$ d'entre eux sont implantés entre $370 \mathrm{~m}$ et $400 \mathrm{~m}, 40 \%$ entre $400 \mathrm{~m}$ et $500 \mathrm{~m}$, soit une altitude moyenne de $419 \mathrm{~m}$. Or, on sait que cette période correspond à une phase de haut niveau relatif du plan d'eau, susceptible d'avoir désorganisé le drainage des zones périphériques.

\section{LE BRONZE FINAL}

Le Bronze final est représenté par 10 sites, 3 indices de sites, 1 nécropole et 1 découverte isolée (fig. 15). 2 sites sont occupés au Bronze final 1 [230, 326], entre 1 et 6 le sont au Bronze final 2 [319, 103 ?, 227 ?, 315 ?, 321 ?, 328 ?] et au maximum 4 au Bronze final 3 [103 ?, 227 ?, 230 ?, 321 ?]. Outre la densification apparente du peuplement, on note, pour la première fois, une occupation du fond de la cuvette de Sarliève au début de la période avec la nécropole tumulaire [CO-081] du Bronze final 1 fouillée par G. Vernet ${ }^{5}$. Cette colonisation relative (car il s'agit de sépultures et non d'habitats) des terres les plus basses pourrait s'expliquer par l'abaissement du plan d'eau, attesté à la fois par les fouilles et par l'étude des microfossiles non polliniques. La zone de l'émissaire présente aussi des indices d'occupation, mais ceux-ci posent des problèmes d'attribution chronologique. On remarque cependant qu'aucun site de la cuvette et des piémonts n'est clairement attribuable au Bronze final 3. En outre, la majorité des établissements se localise, comme aux périodes précédentes, sur les versants du plateau de Gergovie, des puys d'Anzelle et de Bane. Le rebord sud-est du plateau de Gergovie, s'il ne paraît plus occupé au début du Bronze final, est investi de nouveau au Bronze final 2 et continue à l'être au Bronze final 3 (Milcent, 2004). C'est probablement au cours de l'une de ces deux périodes qu'un rempart y est bâti ou reconstruit (information orale: T. Pertlwieser). Le site de la Maison des loisirs à La Roche-Blanche, fouillé par G. Loison, correspond à un habitat dense et étendu implanté au Bronze final 3 sur une haute terrasse.

5. Le site [315], localisé dans le fond de la cuvette, est de toute évidence perturbé.
Il a été observé en Basse-Auvergne que les indices de peuplement présentent une évolution complexe au Bronze final (Milcent, 2002 et 2004). C'est au Bronze final 1 et 2 que des sites gagnent le cœur de la Limagne. Il s'agit d'occupations de faible ampleur et de courte durée correspondant vraisemblablement à des unités agricoles déplacées de manière cyclique. Hormis des nécropoles, les sites du Bronze final 3 ne sont en revanche pas attestés de manière assurée dans les terrains bas. Par contre, et comme à La Roche-Blanche, on trouve sur de hautes terrasses et sur la plupart des reliefs dominant la Limagne des témoignages d'établissements à la fois denses et étendus (puy de Corent, puy de Gravenoire, puy Saint-André).

\section{L'ÂGE DU FER}

\section{LE HALLSTATT ANCIEN ET MOYEN}

Le premier âge du Fer se caractérise par une densification assez nette du peuplement : on compte alors 17 sites et 7 indices de sites (fig. 16). Au Bronze final, 8 sites étaient assurément déjà occupés, mais sans que l'on puisse établir une continuité car il n'existe pas d'exemple d'un établissement ayant livré à la fois du mobilier du Bronze final 3 et du Hallstatt ancien. La localisation des implantations paraît s'inscrire dans la continuité des périodes antérieures, puisque la grande majorité des sites sont établis sur les versants et les piémonts. Toutefois, le sommet du plateau de Gergovie et les terrasses de haut de versant ne sont plus occupés au Hallstatt ancien, sinon pour d'hypothétiques sépultures. On observe en outre que la bordure de la cuvette de Sarliève n'est pas répulsive, puisqu'au moins un site [181] et trois indices de sites y ont été repérés. En d'autres points de la Limagne (Milcent, 2002 et 2004), on constate que le Hallstatt ancien correspond à une véritable colonisation de la plaine par des unités agricoles dispersées (fermes et hameaux ?), après une relative désertion au Bronze final 3. Comme au Bronze final 1 et 2, ces installations de plaine ne livrent pas d'indices d'une occupation de longue durée et paraissent régulièrement déplacées, ce qui peut contribuer à expliquer leur nombre élevé. Ce processus de colonisation est concomitant d'un abandon des habitats groupés de plateaux et de terrasses. Cette rupture dans le schéma de peuplement participe d'un processus qui voit la mise en place d'une nouvelle organisation des sociétés protohistoriques à compter du VIII ${ }^{\mathrm{e}}$ s. av. J.-C. Le Hallstatt moyen s'inscrit en l'état dans cette dynamique initiée au début de l'âge du Fer. 


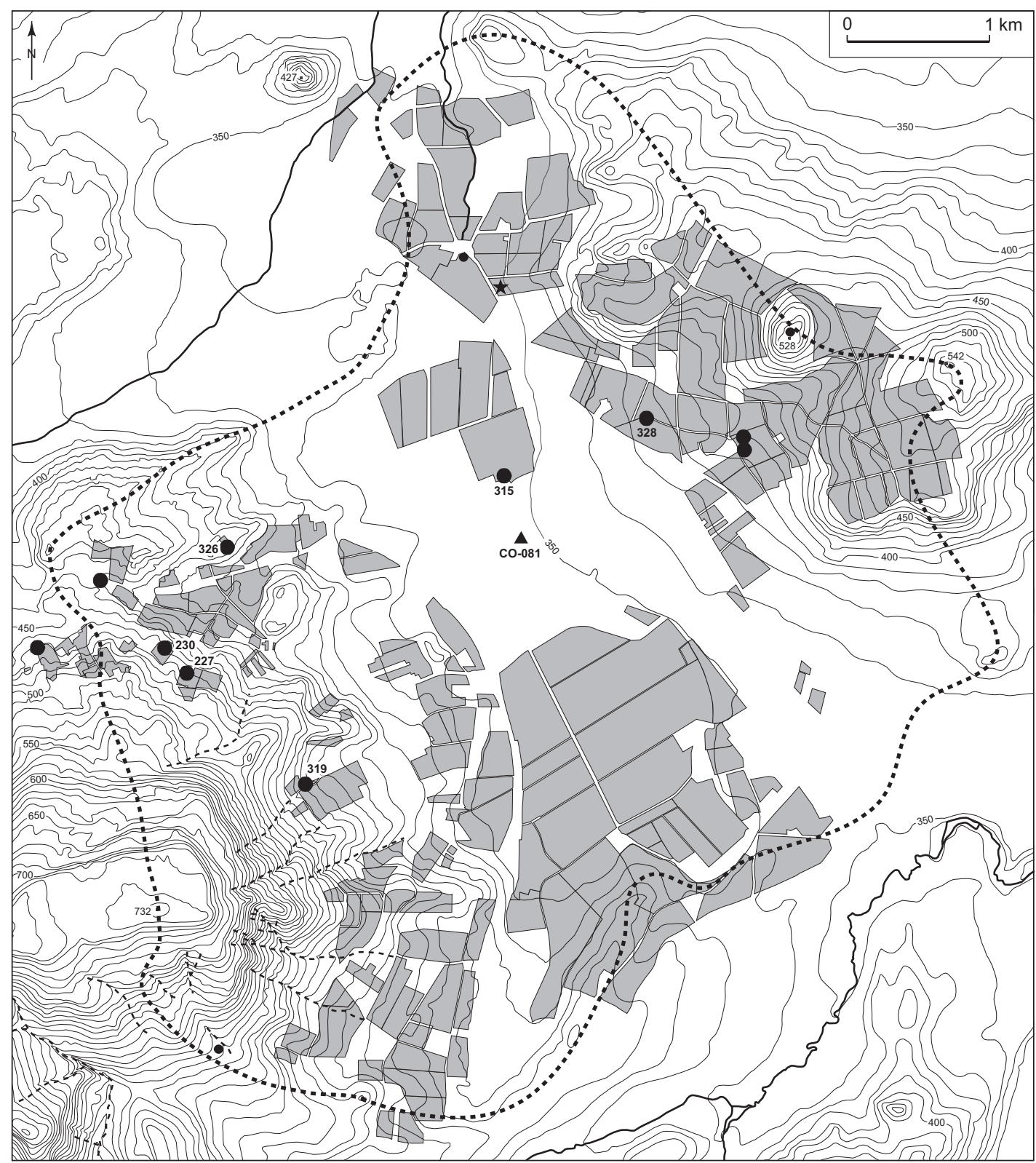

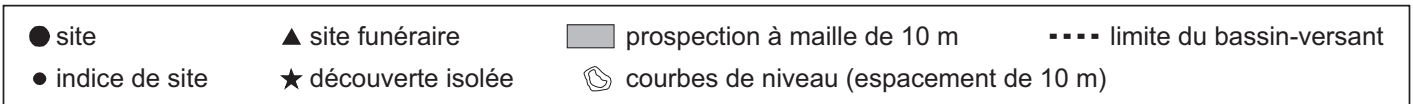

Fig. 15 - L'occupation du bassin de Sarliève au Bronze final (DAO : B. Dousteyssier et F. Trément, Université Clermont-Ferrand-II).

\section{LE HALLSTATT FINAL}

La période du Hallstatt final demeure très mal documentée, à Sarliève comme dans le reste de l'Auvergne, ce qui pose un problème d'interprétation. Les marqueurs chronologiques de cette période sont pourtant suffisamment bien connus par ailleurs. La grande discrétion des implantations datées $\mathrm{du} \mathrm{VI}^{\mathrm{e}} \mathrm{s}$. av. J.-C. en Basse-Auvergne est encore à ce jour le seul constat objectif que l'on puisse faire. Il faut pourtant se garder de conclusions hâtives, comme en témoignent les premiers indices mobiliers d'une occupation de l'oppidum de Gergovie au Hallstatt D2/3 (fouilles T. Pertlwieser, 2001). 


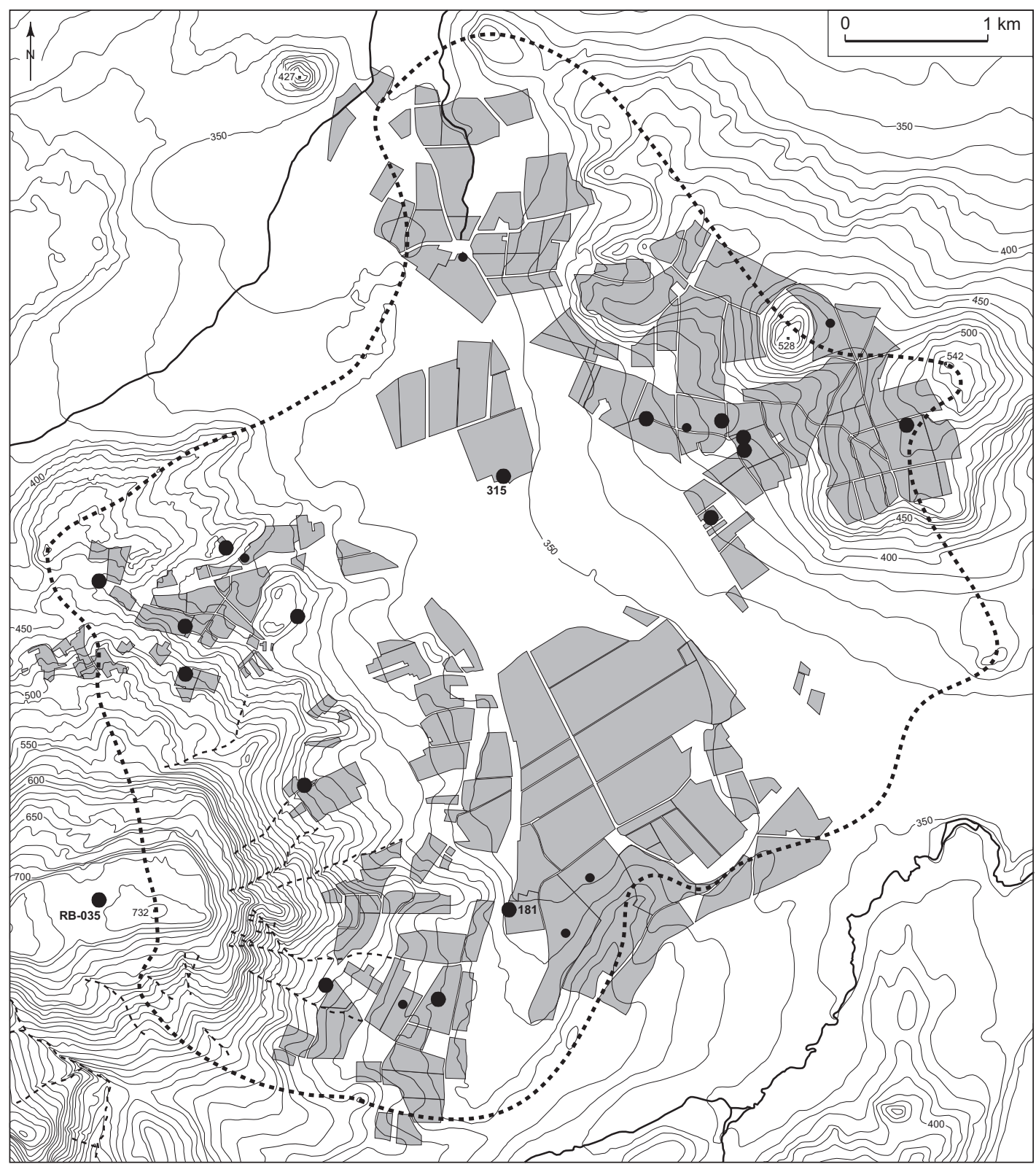

\begin{tabular}{|l|ll|}
\hline site & prospection à maille de $10 \mathrm{~m}$ & $\ldots$ limite du bassin-versant \\
- indice de site & courbes de niveau (espacement de $10 \mathrm{~m}$ ) & \\
\hline
\end{tabular}

Fig. 16 - L'occupation du bassin de Sarliève au premier âge du Fer (DAO : B. Dousteyssier et F. Trément, Université Clermont-Ferrand-II).

\section{LA TÈNE ANCIENNE ET MOYENNE}

On constate pour La Tène ancienne, notamment le $\mathrm{V}^{\mathrm{e}} \mathrm{s}$., un déficit certain dans l'occupation du bassin (fig. 17). L'occupation de La Tène B1 et B2 du site de la Grande Halle
[CO-082], celle de La Tène B2 de la nécropole de Bonnabry [CO-042] et celle du site de la Novialle [RB-023] représentent les seules occurrences datées avec précision du IV ${ }^{\mathrm{e}} \mathrm{s}$. av. J.-C. Les fouilles de la Grande Halle ont révélé un habitat de La Tène B2 [CO-082] et quatre sépultures attribuées à la 


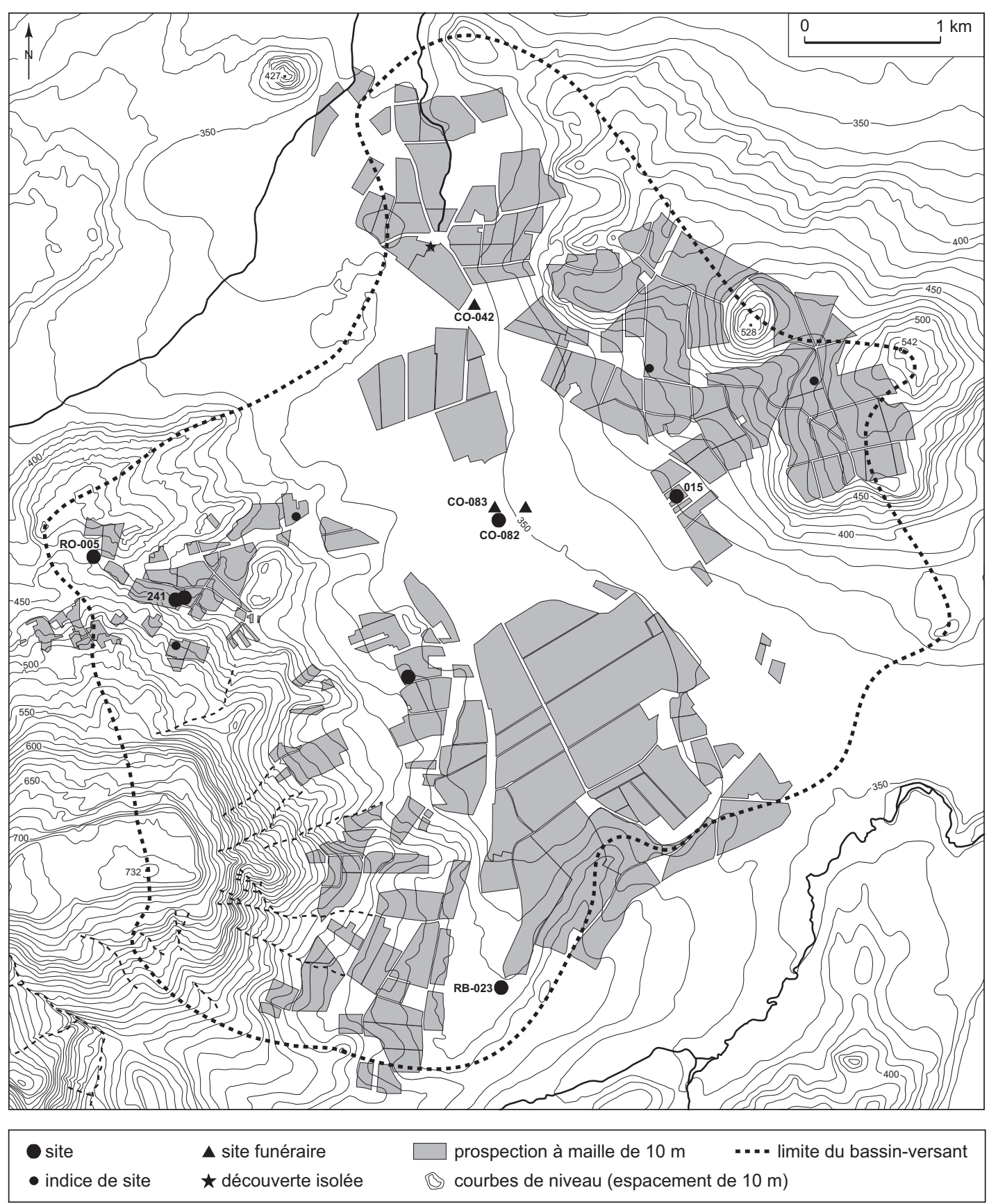

Fig. 17 - L'occupation du bassin de Sarliève à La Tène ancienne (DAO : B. Dousteyssier et F. Trément, Université Clermont-Ferrand-II).

phase de transition La Tène B2/C1 [CO-083]. Les autres données concernant La Tène ancienne, issues des prospections, sont attribuées avec une large approximation chronologique car le mobilier est peu nombreux et très fragmenté. On compte au total 7 sites, 4 indices de sites, 3 nécropoles et 1 découverte isolée. Plusieurs emplacements s'inscrivent dans la continuité des choix d'implantation du premier âge du Fer [RO-005, 015, 241], mais il s'agit davantage d'une récurrence que d'une permanence de l'habitat. Dans l'ensemble, l'occupation concerne de nouvelles terres situées à une plus grande proximité de la cuvette. L'altitude moyenne des établissements, à $387 \mathrm{~m}$ NGF, n’a jamais 


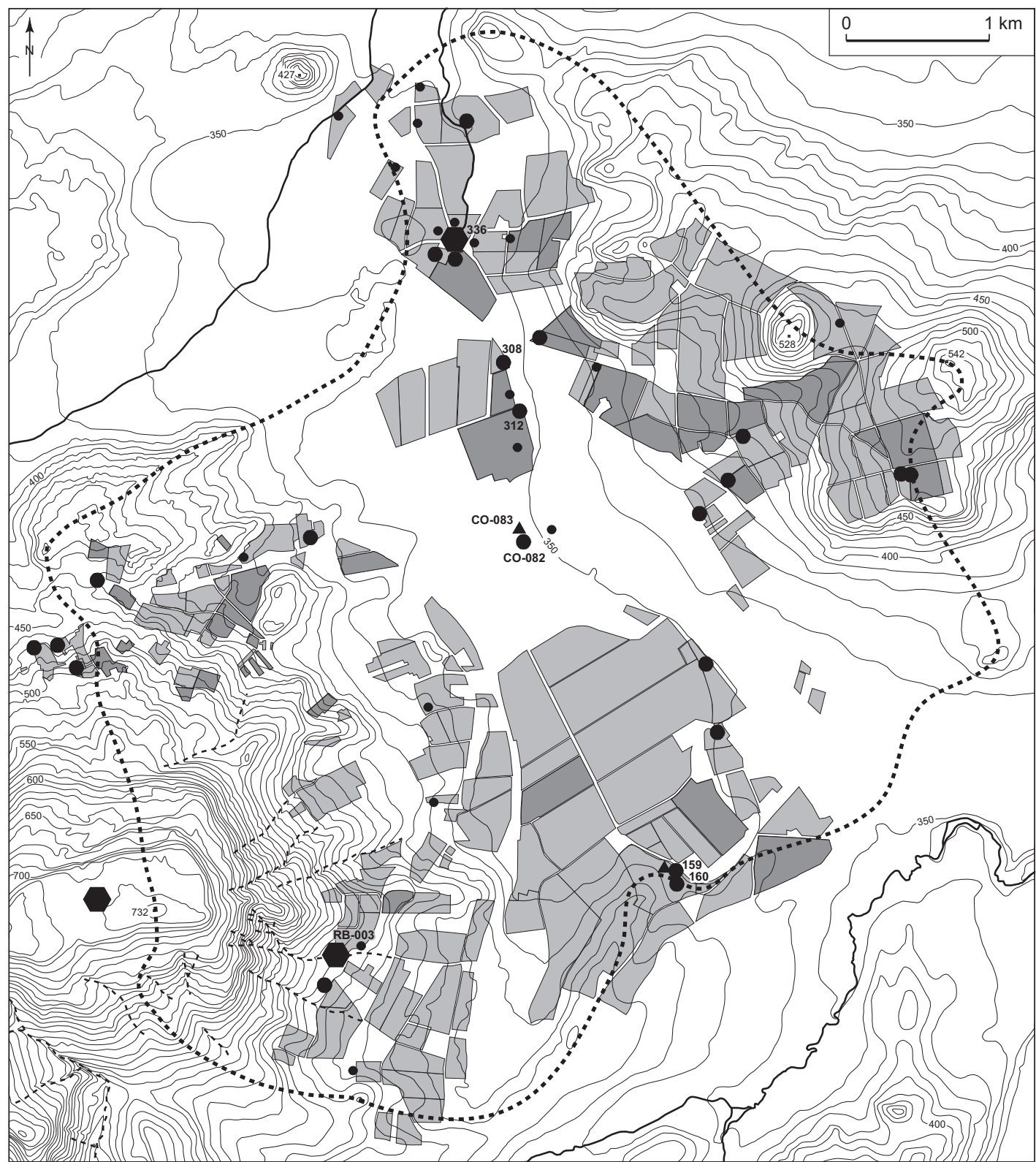

agglomération

site

$\Delta$ site funéraire $\square$ épandage

(c) courbes de niveau (espacement de $10 \mathrm{~m}$ )

Fig. 18 - L'occupation du bassin de Sarliève à La Tène finale (DAO : B. Dousteyssier et F. Trément, Université Clermont-Ferrand-II).

été aussi basse. Ces nouvelles implantations s'alignent le long d'un axe qui longe la limite orientale du marais. On peut mettre ce phénomène en rapport avec l'expansion, au débouché du bassin, du site de la Grande Borne à Aulnat à partir de La Tène B2/C1, soit à l'extrême fin du $\mathrm{IV}^{\mathrm{e}} \mathrm{s}$. ou dans les premières décennies du $\mathrm{III}^{\mathrm{e}} \mathrm{s}$. av. J.-C.

\section{LA TÈNE FINALE}

On observe, à partir du $\mathrm{II}^{\mathrm{e}}$ s. av. J.-C. (La Tène C2/ D1), une très forte densification de l'habitat, puisqu'on ne dénombre pas moins de 24 sites, 15 indices de sites et 2 nécropoles (fig. 18). Quantitativement, cette période marque une véritable rupture dans l'occupation du bassin. 

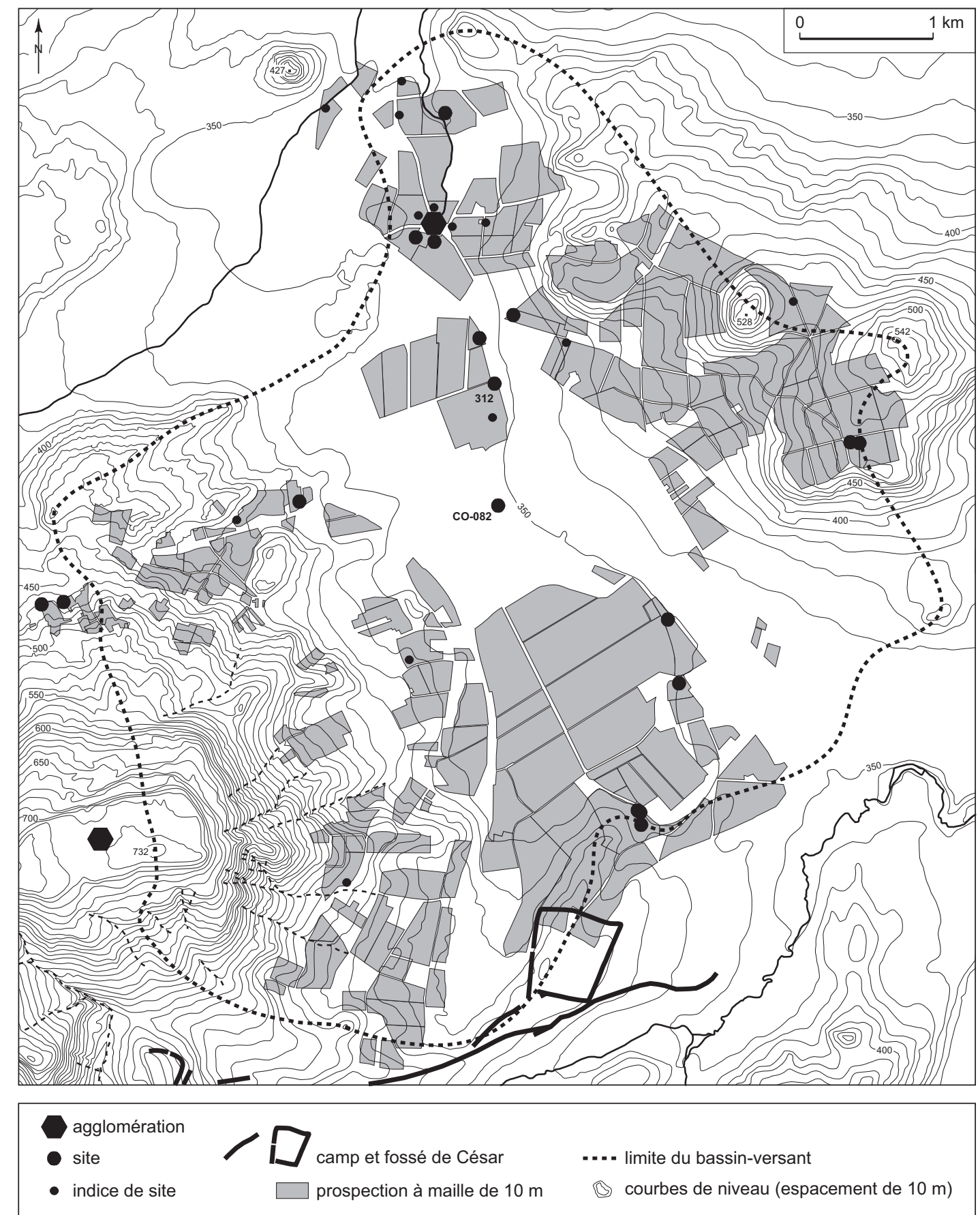

Fig. 19 - L'occupation du bassin de Sarliève au Irr s. av. J.-C. (DAO : B. Dousteyssier et F. Trément, Université Clermont-Ferrand-II).

Le peuplement de la bordure du marais s'inscrit dans la continuité de l'évolution amorcée durant La Tène ancienne et moyenne, même s'il tranche radicalement par le nombre des implantations. De nouveaux établissements s'installent dans la cuvette ou en bordure immédiate, formant une sorte de chapelet sur le bord de la terrasse orientale. Au nord-est, il est probable qu'une zone funéraire se développe $\mathrm{au} \mathrm{II}^{\mathrm{e}} \mathrm{s}$. av. J.-C. en direction du marais [308], peut-être à partir d'un noyau hérité de la fin de La Tène ancienne, la nécropole de Bonnabry [CO-042]. La zone de l'émissaire fait l'objet également d'une occupation dense, en particulier dans le secteur de Belde [336], occupé par intermittence 
depuis le Néolithique. On observe, parallèlement, une densification relative du peuplement sur les versants et les plateaux périphériques.

L'habitat se répartit de manière équilibrée entre les différentes unités physiques : plateaux, versants, piémonts, vallons, terrasse orientale et cuvette. On peut voir là le signe d'une intensification de l'exploitation du milieu, qui s'initie bien avant l'occupation de l'oppidum de Gergovie et fait écho au mouvement général observé dans l'ensemble de la Limagne (Mennessier-Jouannet et al., 2002 ; Trément et al., 2002).

Les fouilles de la Grande Halle corroborent l'hypothèse d'une pression accrue des communautés riveraines sur la bordure orientale du marais, qui est alors très largement exondé (fig. 18). Sur la bordure méridionale de la cuvette, au lieu-dit l'Enfer [159/160], les fouilles préalables à l'aménagement du contournement sud-est de Clermont-Ferrand ont révélé la présence d'une ferme indigène associée à quelques inhumations (fouille Baucheron, 2001). L'enclos quadrangulaire à grand fossé, d'une superficie d'environ $4000 \mathrm{~m}^{2}$, était doté d'une entrée aménagée sans doute avec un portique (information orale: Y. Deberge). Dans son comblement ont été recueillies des moules d'eau douce. L'ensemble est occupé au plus tôt au milieu du $\mathrm{II}^{\mathrm{e}} \mathrm{s}$. av. J.-C. et abandonné antérieurement à la fin de ce siècle. Toutefois, comme sur le site de la Grande Halle, le secteur reste occupé au siècle suivant et donne lieu à l'installation d'un établissement gallo-romain.

L'occupation du bassin reste dense à La Tène D2 (fig. 19). On compte alors 17 sites et 10 indices de sites, localisés préférentiellement dans les zones les plus basses (53\% sont implantés à moins de $350 \mathrm{~m}$ d'altitude). La cartographie des épandages révèle une étroite association entre habitat et zones potentiellement amendées. Il semble bien que cette période corresponde à une réorganisation foncière, qui crée les conditions du développement de l'économie domaniale attesté dès le début du siècle suivant. Le parcellaire borné daté de La Tène finale, mis au jour dans le fond de la cuvette de Sarliève lors du diagnostic de la Grande Halle, est peutêtre le signe tangible de cette restructuration du paysage par les élites possédantes (Trément, Dousteyssier, 2003).

\section{LE HAUT-EMPIRE}

\section{DOMESTICATION DU MILIEU}

Durant le Haut-Empire, la densité de l'habitat révélée par les prospections est nettement supérieure à celle de l'âge du Fer, puisqu'on dénombre 57 sites, 31 indices de sites et 11 nécropoles (fig. 20). Les deux premiers siècles de notre ère se caractérisent par une généralisation de la mise en valeur à l'ensemble du bassin, quels que soient les types de milieux : sommets, versants, piémonts, vallons, bordure, émissaire et fond de la cuvette. $40 \%$ des sites sont implantés à moins de $350 \mathrm{~m}$ d'altitude, $30 \%$ entre $350 \mathrm{~m}$ et $400 \mathrm{~m}$, $28 \%$ entre $400 \mathrm{~m}$ et $500 \mathrm{~m}, 2 \%$ à plus de $700 \mathrm{~m}$. L'altitude moyenne des établissements, à $390 \mathrm{~m}$, est particulièrement faible.

La cartographie des sites et des épandages montre que deux types de milieux font l'objet d'une mise en valeur plus intense qu'auparavant : d'une part, les sommets des collines marno-calcaires orientales; d'autre part, la bordure et le fond de la cuvette de Sarliève. La zone funéraire septentrionale connaît une nouvelle extension vers le milieu du paléolac [308, 309, 310, 311]. La présence de plusieurs établissements, non seulement sur le delta sableux [CO-084, CO-085, CO-086], mais aussi en contrebas [312], prouve que l'assèchement du marais est presque complet, tout au moins dans sa partie nord. Dans la cuvette méridionale, l'implantation systématique des établissements sur la terrasse orientale pourrait indiquer que ce secteur était moins bien drainé, ou saisonnièrement engorgé.

\section{TYPOLOGIE DE L'HABITAT}

La typologie de l'habitat dispersé peut être appréhendée avec davantage de finesse qu'auparavant pour cette période. Trois classes de sites ont été établies sur des critères qualitatifs : la classe A regroupe les établissements sur lesquels des fragments de marbre et/ou des tesselles de mosaïque ont été recueillis ; la classe B réunit les sites présentant des traces d'enduits peints et/ou des vestiges d'hypocauste; enfin, la classe $\mathrm{C}$ rassemble le reste des implantations.

\section{Classe A}

La classe A se compose de 9 établissements. Leur superficie moyenne s'élève à $12550 \mathrm{~m}^{2}$, si l'on exclut le site de Belde [336], dont les dimensions exceptionnelles (20 ha) s'expliquent par la prise en compte de l'ensemble constitué par la pars urbana et les bâtiments annexes. Elle est généralement comprise entre $10000 \mathrm{~m}^{2}$ et $25000 \mathrm{~m}^{2}$. Leur altitude moyenne est d'à peine 360 m NGF. Ces sites se répartissent de manière très régulière, en chapelet, sur le pourtour du bassin et dans le vallon de Maréchal, avec un espacement de $1500 \mathrm{~m}$ à $1800 \mathrm{~m}$. 


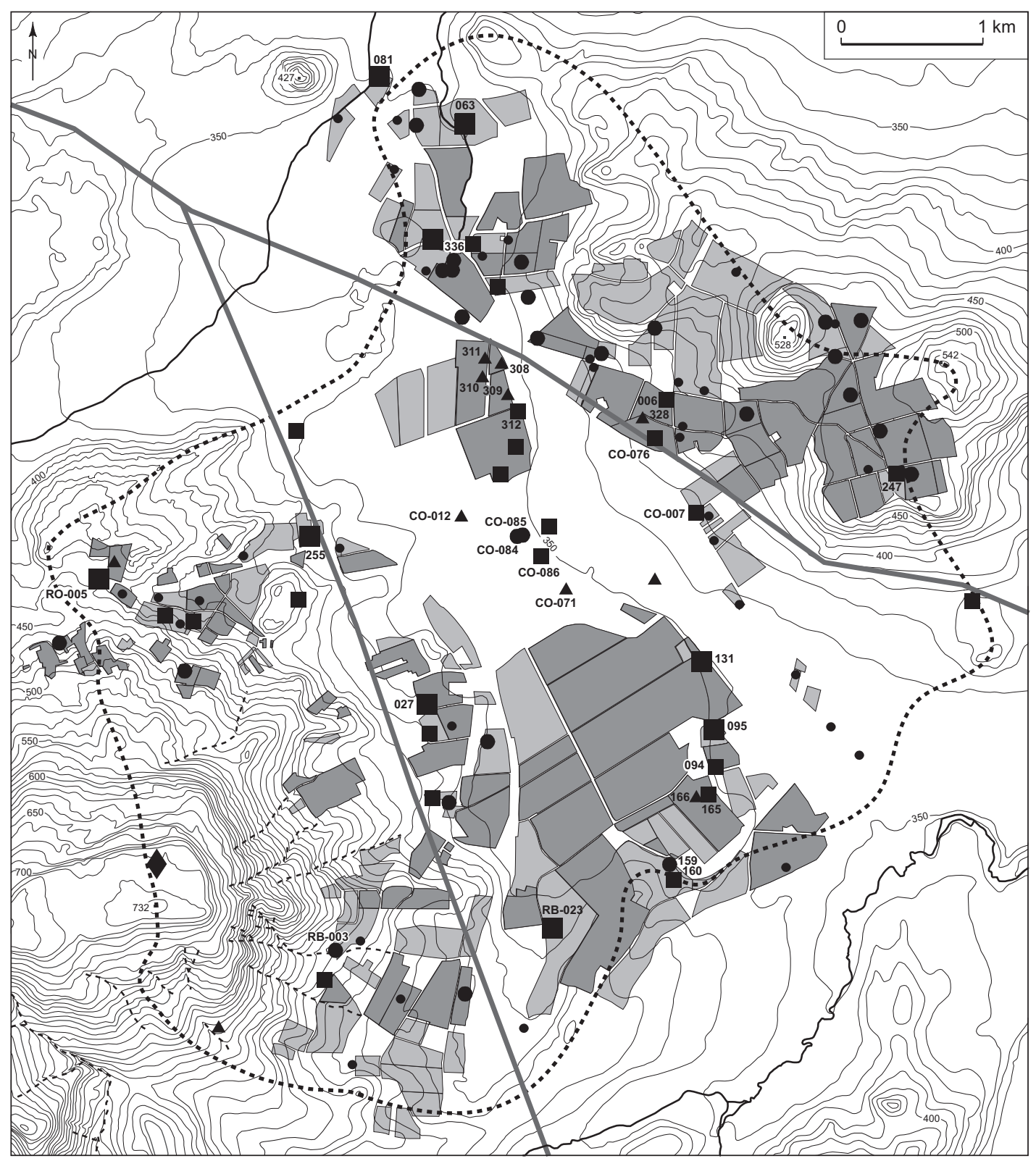

site de rang $A$

- site de rang $B$

site de rang $C$

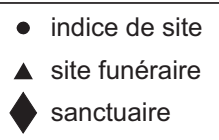

$\square$ prospection à maille de $10 \mathrm{~m}$

... - limite du bassin-versant

$\square$ épandage

(c) courbes de niveau (espacement de $10 \mathrm{~m}$ )

Fig. 20 - L'occupation du bassin de Sarliève au Haut-Empire (DAO : B. Dousteyssier et F. Trément, Université Clermont-Ferrand-II).

Une autre caractéristique des établissements de la classe A est leur longévité. Sur les 9 sites concernés, 7 étaient occupés à La Tène finale, 8 le sont encore au Bas-Empire et 5 présentent des indices du haut Moyen Âge. Il est clair que, comme dans le reste de la Grande Limagne, ce type d'éta- blissement a structuré durablement les campagnes dans le bassin de Clermont-Ferrand (Trément, Dousteyssier, 2003 ; Dousteyssier et al., 2004). Tous ces arguments suggèrent qu'il s'agit là des centres de vastes exploitations domaniales appartenant à des notables de la cité arverne (Trément, 2002). 


\section{Classe B}

La superficie moyenne des 22 sites de la classe B s'élève à $5190 \mathrm{~m}^{2}$. Elle est généralement comprise entre $2500 \mathrm{~m}^{2}$ et $10000 \mathrm{~m}^{2}$. Ces sites s'intercalent entre ceux de la classe précédente, dont ils sont parfois très proches. On les rencontre principalement sur la bordure du paléolac, dans le vallon de Maréchal, sur les piémonts du puy d'Anzelle et du plateau de Gergovie. Leur altitude moyenne est de 377 m NGF. L'absence de tout indice de luxus, à l'exception de modestes enduits peints dans quelques cas, invite à y voir des exploitations agricoles ou des bâtiments dépendant des villae de la classe A. Ces établissements présentent fréquemment des indices de continuité avec le Bas-Empire, moins souvent avec La Tène finale ou le haut Moyen Âge. Sur 22 sites, 7 sont occupés dès La Tène finale, 8 dès la première moitié du $\mathrm{I}^{\mathrm{er}}$ s. apr. J.-C. ; 15 sont occupés au Bas-Empire, 2 seulement au haut Moyen Âge. Au moins 4 sites sont occupés continûment de La Tène finale au Bas-Empire, et 1 de La Tène finale au Moyen Âge. La capacité de ces établissements à structurer durablement le paysage est donc moins forte que celle des sites de la classe A.

\section{Classe C}

Les sites de la classe $\mathrm{C}$, au nombre de 23, ont une superficie moyenne de $2193 \mathrm{~m}^{2}$. Les surfaces mesurées au sol n'excèdent jamais $4500 \mathrm{~m}^{2}$. Dans 6 cas, elles sont inférieures à $1000 \mathrm{~m}^{2}$. Ces implantations modestes, dispersées dans tout le bassin, se rencontrent aussi bien dans le voisinage des établissements de classes A et B que dans des secteurs où ces derniers sont absents : c'est le cas, en particulier, sur les sommets et les versants marno-calcaires des puys de Bane et d'Anzelle où leur maillage régulier témoigne d'une occupation méthodique de zones jusqu'alors délaissées. On pense à de modestes fermes ou à des annexes agricoles. Leur altitude moyenne, à 403 m NGF, est nettement plus élevée que celle des établissements de classe A ou B. Ces implantations sont directement liées au mouvement de conquête de nouvelles terres qui caractérise le $\mathrm{I}^{\mathrm{er}} \mathrm{s}$. de notre ère. Elles sont pour la plupart abandonnées dans le courant du $\mathrm{III}^{\mathrm{e}} \mathrm{s}$.

\section{LE BAS-EMPIRE}

Dans le courant du III $^{\mathrm{e}}$ s., peut-être dès avant le milieu de ce siècle, l'habitat dispersé connaît un resserrement très net autour des établissements les plus importants (fig. 21). Le nombre total de sites tombe de 54 à 17 , soit une réduction de plus de $68 \%$. Mais cette diminution affecte très inégalement les différentes classes d'implantations : en effet, sur 9 sites de rang A occupés au Haut-Empire, 8 présentent des indices du Bas-Empire (soit $89 \%$ ) ; c'est le cas de 15 sites de rang B sur $22(68 \%)$ et de seulement 2 sites de rang C sur 23 (9\%). Aucun site n'est créé durant cette période. La part des établissements de rang A passe ainsi de $17 \%$ à $29 \%$, celle des implantations de rang B de $41 \%$ à $59 \%$ et celle des sites de rang $\mathrm{C}$ de $42 \%$ à $12 \%$. Le même phénomène est observé dans toute la Grande Limagne (Trément, Dousteyssier, 2003, p. 666).

Ces valeurs sont comparables à celles de Gaule Narbonnaise, où l'hypothèse d'un vaste mouvement de concentration foncière a été avancée (Fiches dir., 1996). Pourtant il n'existe, en Auvergne, aucun exemple de sites dont la taille augmenterait entre le Haut- et le Bas-Empire. La fouille de la villa de Maréchal [RO-005] montre même le contraire. Abandonné dans le dernier quart du $\mathrm{III}^{\mathrm{e}}$ s., cet établissement n'est réoccupé que de manière limitée dans la seconde moitié du $\mathrm{IV}^{\mathrm{e}} \mathrm{s}$. et au début du $\mathrm{V}^{\mathrm{e}} \mathrm{s}$., perdant de toute évidence son statut privilégié (Fourvel, Liegard, 1996).

La majorité des sites se localise dans les zones basses (piémonts, vallon de Maréchal, bordure et émissaire de la cuvette). Leur altitude moyenne, à 383 m NGF, n'a jamais été aussi faible. L'altitude moyenne des établissements de la classe A est de $352 \mathrm{~m}$, celle des implantations de la classe B de $392 \mathrm{~m}$ et celle des sites de la classe C de $416 \mathrm{~m}$. Ces tendances pourraient traduire une reconversion des versants et des plateaux qui avaient été conquis à l'agriculture durant le Haut-Empire, peut-être au profit de l'élevage ou de la viticulture. Au V ${ }^{\mathrm{e}}$ s., Sidoine Apollinaire décrit une plaine intensément cultivée. Il évoque un «océan de blés ", source de profit pour les laboureurs, ainsi que des vignobles sur les coteaux et une ceinture de pâturages au sommet des montagnes (Epistulae, IV, 21).

\section{LE HAUT MOYEN ÂGE}

Les tendances qui caractérisent l'évolution de l'habitat au Bas-Empire se perpétuent durant le haut Moyen Âge : permanence de l'habitat dispersé, érosion différentielle du nombre d'établissements en fonction de leur rang, localisation préférentielle dans les zones basses. Pour cette période globalement mal connue du point de vue archéologique, le bilan des prospections n'est pas négligeable: 5 sites, 4 indices de sites, 2 sites funéraires et 2 découvertes isolées (fig. 22). Sur 5 établissements de rang A occupés au 


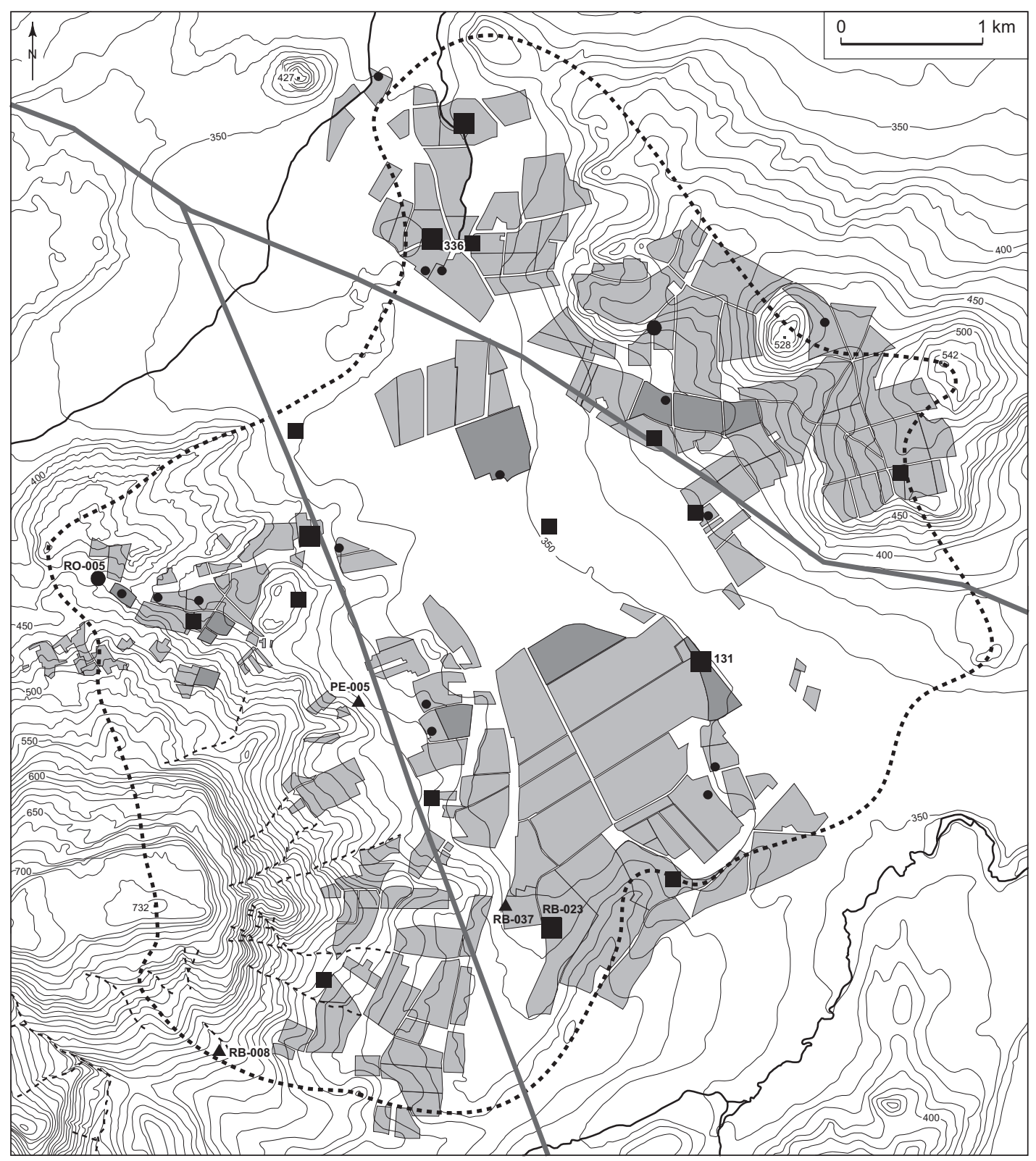

site de rang $A$

- indice de site

$\square$ prospection à maille de $10 \mathrm{~m}$

.... limite du bassin-versant

- site de rang $B$

- site funéraire

épandage

(c) courbes de niveau (espacement de $10 \mathrm{~m}$ )

site de rang $C$

Fig. 21 - L'occupation du bassin de Sarliève au Bas-Empire (DAO : B. Dousteyssier et F. Trément, Université Clermont-Ferrand-II).

Bas-Empire, 4 présentent des indices du haut Moyen Âge. En revanche, sur 10 implantations de rang B, 2 seulement paraissent encore occupées. Aucun des 2 sites de rang C n’a livré de témoins de cette période.

Les 4 sites de rang A en question sont occupés de manière continue depuis La Tène finale : il s'agit du vaste domaine de Belde [336] (fig. 22 et 23), de la grosse villa du Pré du Camp [255] et des deux établissements de la gare de Sarliève [131] et du Grand Beaulieu [063]. On notera avec intérêt que 3 de ces sites donnent naissance à d'importants domaines seigneuriaux au Moyen Âge [336, 131, 063]. 


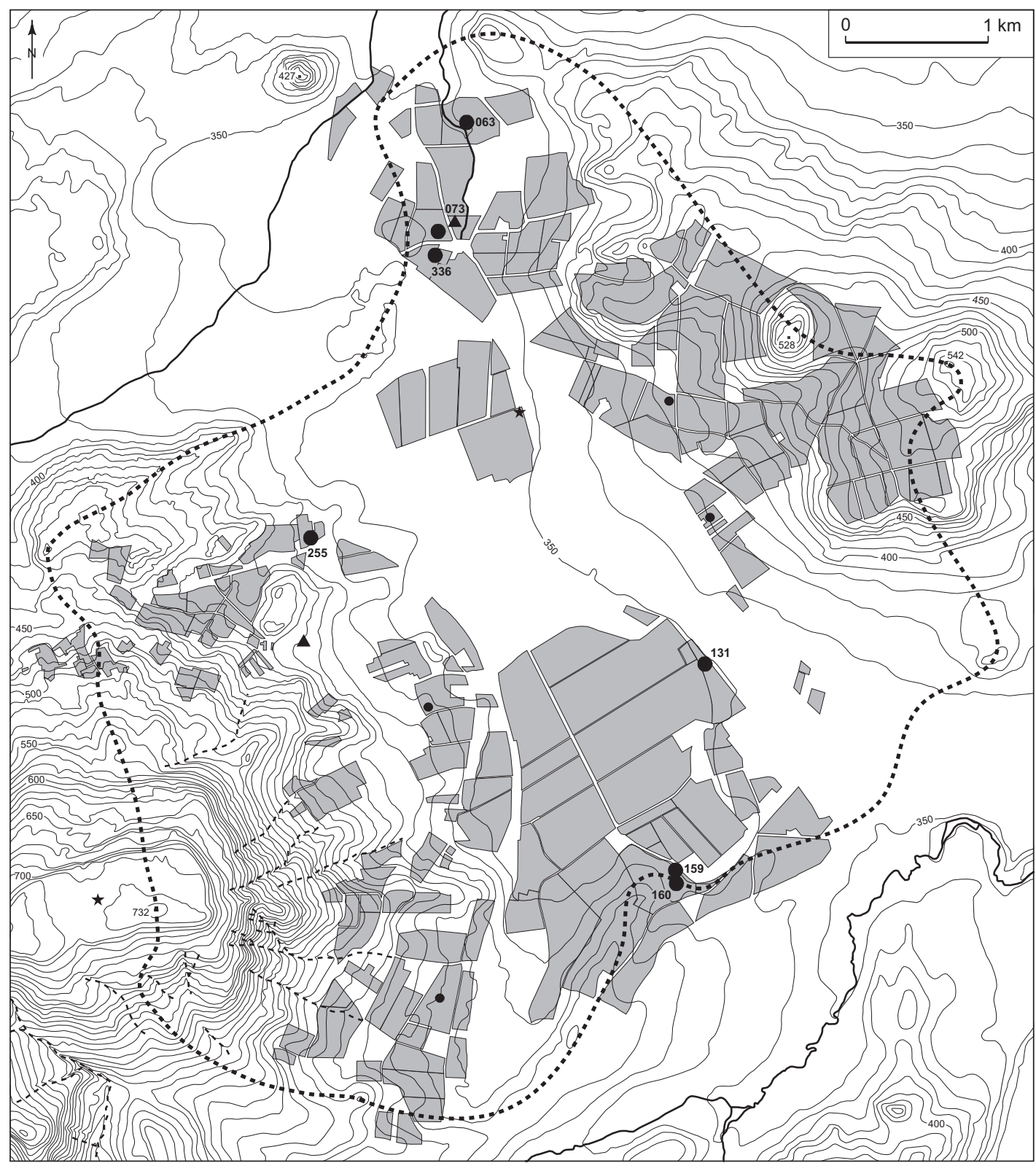

\begin{tabular}{|c|c|c|}
\hline site & $\Delta$ site funéraire & $\square$ prospection à maille de $10 \mathrm{~m}$ \\
\hline - indice de site & $\star$ découverte isolée & (c) courbes de niveau (espacement de $10 \mathrm{~m}$ ) \\
\hline
\end{tabular}

Fig. 22 - L'occupation du bassin de Sarliève au haut Moyen Âge (DAO : B. Dousteyssier et F. Trément, Université Clermont-Ferrand-II).

L'altitude moyenne de ces établissements $(351 \mathrm{~m})$ est plus faible que jamais. En effet, ils se localisent sur la bordure du marais de Sarliève et à son débouché. Leur distribution dans l'espace pourrait refléter un partage équilibré du bassin entre quelques grands domaines. La localisation des indices de sites trahit quant à elle l'existence d'un petit habitat dispersé sur les piémonts. En revanche, plateaux et versants semblent être désertés. Ils étaient peutêtre voués davantage aux troupeaux qu'aux cultures. On note d'ailleurs une nette rétraction des zones d'épandage par rapport au Haut-Empire.

Les données archéologiques corroborent le témoignage de Sidoine Apollinaire et de Grégoire de Tours sur la persistance de grands domaines en Limagne aux $\mathrm{V}^{\mathrm{e}}$ et $\mathrm{VI}^{\mathrm{e}} \mathrm{s}$. 


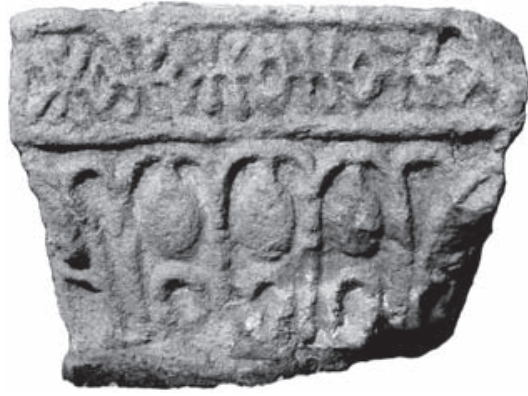

a

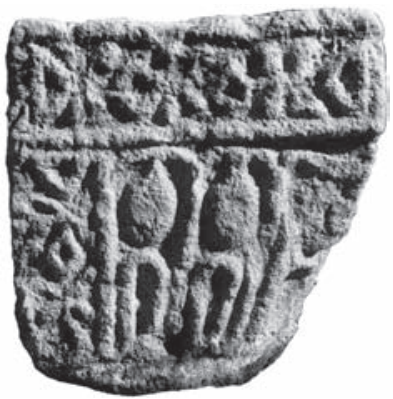

b

Fig. 23 - Chapiteau de colonne engagée d'époque carolingienne tardive ou romane précoce, découvert à l'emplacement de la pars urbana de la villa de Belde : a, face avant ; b, côté gauche (clichés : F. Trément, Université Clermont-Ferrand-II).

La famille de l'évêque d'Arvernie possédait d'importants biens fonciers en Auvergne au $\mathrm{V}^{\mathrm{e}} \mathrm{s}$. (Sidoine Apollinaire, Epistulae, II, 2 et 12 ; VIII, 9). Un siècle plus tard, Grégoire de Tours signale de grands domaines ruraux sur lesquels la famille des propriétaires séjournait régulièrement (Grégoire de Tours, Liber in gloria martyrum, 83).

\section{L'OCGUPATION DU FOND DE LA CUVETTE PENDANT L'ANTIQUITÉ}

Dans la partie septentrionale du paléolac, en quatre points [308, 309, 310, 311, fig. 20] situés au fond de la cuvette, aux environs de la cote 345 m NGF, les ramassages ont livré de la céramique fine brûlée datée des deux premiers siècles de notre ère, associée à un ou deux fragments d'objets en bronze, ainsi que des fragments d'amphore italique. Deux de ces points [308, 309] ont également livré de la céramique de La Tène moyenne et finale. Les assemblages de mobilier sont caractéristiques d'une nécropole à incinération du Haut-Empire. Ces découvertes corroborent les observations effectuées en 1925 par A. Vergnette, qui décrit une nécropole à incinération gallo-romaine [CO-012, fig. 20]
« en pleine cuvette de l'ancien lac de Sarliève " (Vergnette, 1927, p. 266).

La relation faite par P.-P. Mathieu au XIX ${ }^{\mathrm{e}}$ s. prend ainsi un intérêt nouveau : «On a précisément exhumé, il y a peu de temps, dans le champ de la Gravière, près de la gare de Sarliève, des ossements d'hommes et de chevaux en grande quantité, des têtes humaines encore armées de leurs rateliers blanc comme de l'ivoire, des urnes de formes diverses; des vases en terre rouge, de différentes grandeurs ; une poignée d'épée en cuivre ; un bracelet d'airain encore adhérent à l'os du poignet. Ces épaves d'un autre âge se trouvent, à ce qu'il paraît, dispersées ça et là, sans régularité, sous le sol, et dans une fort grande étendue " (Mathieu, 1862, p. 74).

Dans la partie centrale du paléolac, sur sa bordure orientale, des rejets d'incinérations [CO-071, fig. 20] datés de la période augustéenne ont été identifiés en 2001 dans un fossé, à l'occasion d'un diagnostic archéologique préalable à la construction d'un complexe hôtelier (Cabézuelo, 2001).

Dans la cuvette méridionale, une petite nécropole à incinération gallo-romaine [166, fig. 20] a été identifiée en prospection dans l'anse formée par le paléolac à son extrémité sud-est, aux environs de la cote 344 m NGF. Sur environ $600 \mathrm{~m}^{2}$ se concentraient de nombreux fragments brûlés de céramique fine $\mathrm{du} \mathrm{II}^{\mathrm{e}} \mathrm{s}$., principalement de la sigillée, au répertoire caractéristique (assiettes, coupelles, bols). Cette petite nécropole peut être mise en relation avec l'établissement de rang B [094], distant de $260 \mathrm{~m}$ à peine, implanté au nord-est sur la terrasse qui domine le fond de la cuvette d'environ $2 \mathrm{~m}$.

\section{LES ÉTABLISSEMENTS NON FUNÉRAIRES}

Les sites non funéraires repérés dans la cuvette ellemême sont rares, et l'aspect du matériel, le plus souvent roulé et concrétionné, suggère un déplacement, d'autant que ces sites sont généralement localisés en contrebas d'un delta sableux [165, 314, 315, fig. 20]. Le site [312] fait toutefois exception. Situé en bordure sud de la zone funéraire [308, 309, 310, 311], il se présente sous la forme d'une importante concentration d'artéfacts de grande taille, parfaitement conservés, remontés à la surface par des travaux de curage du fossé de ceinture. Ce mobilier atteste une occupation continue entre La Tène moyenne et le milieu du $\mathrm{I}^{\mathrm{er}} \mathrm{s}$. de notre ère, démontrant que la bordure de la cuvette était alors habitable. La cartographie des épandages confirme que la majeure partie de la dépression était mise en valeur $\mathrm{au} \mathrm{I}{ }^{\mathrm{er}}$ s. av. J.-C. et durant les deux siècles suivants.

F. T., B.D., R.L., G.L., C.M.-J., P.-Y.M., Y.R. 


\section{LES FOUILLES DE LA GRANDE HALLE D'AUVERGNE : ARCHÉOLOGIE D'UN PALÉOLAC}

Les opérations archéologiques conduites dans la partie nord du bassin de Sarliève dans le cadre de l'opération «Grande Halle d'Auvergne » (Vernet et al., 2005) permettent de préciser la nature de l'occupation de la bordure orientale de la cuvette (fig. 5 et pl. II, hors texte).

\section{PREMIERS INDICES D'UNE OCCUPATION HUMAINE AU PALÉOLITHIQUE}

Une vingtaine d'objets lithiques ont été récoltés en position remaniée dans des niveaux sablo-limoneux qui appartiennent à la partie sommitale du cône deltaïque ancien oriental. Leur présence dans ces sédiments montre de façon claire que les bordures de la dépression de Sarliève ont été fréquentées par l'homme préhistorique. L'analyse typologique de ces artéfacts permet de les rapprocher soit d'une industrie de la fin du Paléolithique supérieur (Magdalénien), pour la majorité d'entre eux, soit du début de l'Épipaléolithique pour certaines pièces. Ces données confirment les découvertes d'industries et de faunes préhistoriques faites au XIX ${ }^{\mathrm{e}} \mathrm{s}$. dans des sables en bordure septentrionale de la dépression (Pomel, 1853 ; Pommerol, 1877).

\section{UNE POSSIBLE OCCUPATION AU NÉOLITHIQUE}

Aucun vestige ne peut être clairement rattaché au Néolithique. En revanche, il n'est pas exclu qu'une partie des structures en creux (alignements de trous de poteau et fosses périphériques), fouillées sur la bordure occidentale lors de la phase d'évaluation, remonte à cette période (fig. 5, zone 2). En tout état de cause, ces structures sont antérieures à des systèmes de fossés et de palissades datés de l'âge du Fer. Elles n'ont malheureusement livré aucun mobilier hormis un éclat de silex. Ces trous de poteau s'organisent en deux ensembles formant des structures allongées rectangulaires, qui ne sont pas sans rappeler de longs bâtiments sur poteaux connus par exemple dans le Néolithique ancien septentrional. La plus imposante de ces structures formait un rectangle de $40 \mathrm{~m}$ x $5 \mathrm{~m}$.

\section{LA NÉGROPOLE DU BRONZE FINAL}

La fouille réalisée sur la bordure orientale de la dépression a mis en évidence une occupation durant l'âge du Bronze (fig. 5, zone 1 et pl. II, hors texte). Il s'agit d'un ensemble de neuf structures mégalithiques réparties suivant une bande de terrain de $80 \mathrm{~m}$ de longueur, d'orientation nord-sud, parallèlement au paléorivage [CO-081, fig. 15], qui peuvent être interprétées comme des coffres sépulcraux, malgré l'absence d'ossements humains liée vraisemblablement à la réoccupation du site à La Tène B/C1.

L'une des structures a livré un lot de céramiques caractéristique du Bronze final 1, ce qui conduit à dater ces coffres de cette période. On ne peut cependant pas exclure qu'ils soient plus anciens et que ce lot de céramiques représente déjà une réutilisation de la chambre funéraire. Dans les blocs utilisés pour la construction des coffres, il faut signaler le remploi de meules (entières ou en fragments) et de grandes dalles portant des gravures (cupules et traits), qui peuvent être interprétées comme des fragments de stèles. Dans un cas, il est possible que le coffre mégalithique ait occupé, à l'origine, la partie centrale d'un tumulus qui aurait disparu lors des réutilisations à La Tène. Cette nécropole constitue un précieux marqueur de l'extension maximale du plan d'eau au Bronze final.

\section{L'OCCUPATION DU SECOND ÂGE DU FER}

Les fouilles n'ont livré aucun indice du premier âge du Fer. En revanche, l'occupation attribuable au second âge du Fer est de loin la plus importante (fig. 5, zone 1 et pl. II, hors texte). Les vestiges de cette époque occupent une bande de terrain de $80 \mathrm{~m}$ de largeur et plus de $300 \mathrm{~m}$ de longueur, dans la zone du paléorivage oriental. En plus de cette zone de concentration de vestiges, des fossés de parcellaire ont été repérés au cœur du bassin.

\section{UNE OCGUPATION FORTEMENT STRUCTURÉE}

La fouille d'environ $16000 \mathrm{~m}^{2}$ montre que toute cette zone de tombant, comprise entre le replat de la terrasse alluviale ancienne et le fond de la dépression, a été intensément occupée durant le second âge du Fer, alors que le paléolac était extrêmement réduit et avait laissé hors d'eau ce secteur. Le plan d'ensemble des vestiges révèle une organisation complexe en enclos coalescents dont les fossés principaux adoptent deux orientations principales (pl. II, hors texte) : l'une, nord-ouest/sud-est, parfaitement parallèle au paléorivage, l'autre, nord-est/sud-ouest, perpendiculaire à la première. L'espace se trouve ainsi cloisonné. Le fossé le plus occidental, qui limite le site archéologique proprement dit du fond de la dépression, a pu être reconnu sur plus de $300 \mathrm{~m}$, témoignant d'une forte structuration de l'espace. 


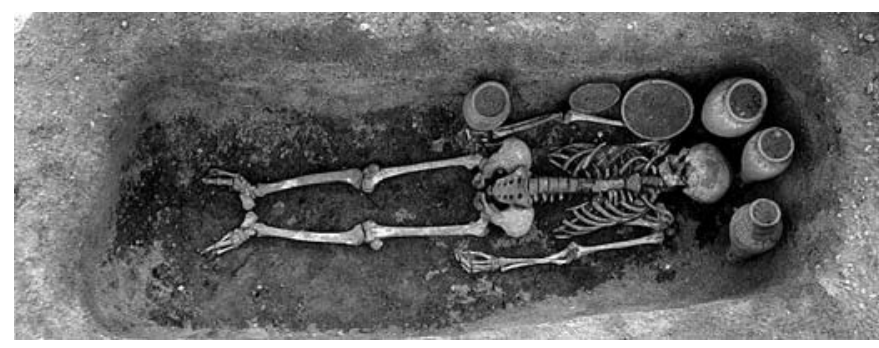

Fig. 24 - La sépulture F2234 de La Tène D1a mise au jour dans le bassin de Sarliève (cliché : G. Vernet, INRAP Clermont-Ferrand).

\section{LA PHASE ANCIENNE DE L'OCCUPATION LATÉNIENNE : LA TÈNE B}

Plusieurs fosses contenant un sédiment cendreux sombre ont livré de la céramique de La Tène ancienne au sens large et/ou de La Tène B [CO-082, fig. 17]. Souvent, ces fosses viennent perturber les structures funéraires de l'âge du Bronze. Le sédiment qui les comble s'est révélé très riche en restes carpologiques. On note également la présence de coquilles d'œufs (poule et oie) et des restes d'anguilles. Il semble qu'il s'agisse de rejets provenant de foyers domestiques. Il n'est pas possible de rattacher à cette période un fossé du site; il faut cependant noter qu'au moins l'un d'entre eux contenait un lot de céramiques de La Tène $B / C$, ce qui tendrait à montrer que certains des fossés présents sur le site ont été créés précocement (à la fin de La Tène B). Dans le même ordre d'idée, plusieurs palissades ou fossés palissadés présents dans la partie nord du décapage et vierges de mobilier céramique pourraient appartenir à la première phase de structuration de l'espace. Enfin, une sépulture d'immature a pu être datée de La Tène B grâce à la présence de deux fibules en fer. Trois sépultures d'adultes ont été attribuées à La Tène B2/C1 sur la foi du mobilier céramique épars collecté dans le comblement de la fosse [CO-083, fig. 17 et 18].

\section{LA PHASE PRINCIPALE DE L'OCCUPATION LATÉNIENNE : LA TÈNE C ET D}

\section{L'habitat}

Si l'on considère les résultats des études de mobilier réalisées par C. Mennessier-Jouannet, une majorité de structures a livré des lots de céramique caractéristiques de La Tène C2/D1. Le site correspond alors à la limite occidentale d'un secteur d'habitat [CO-082, fig. 17, fig. 18 et

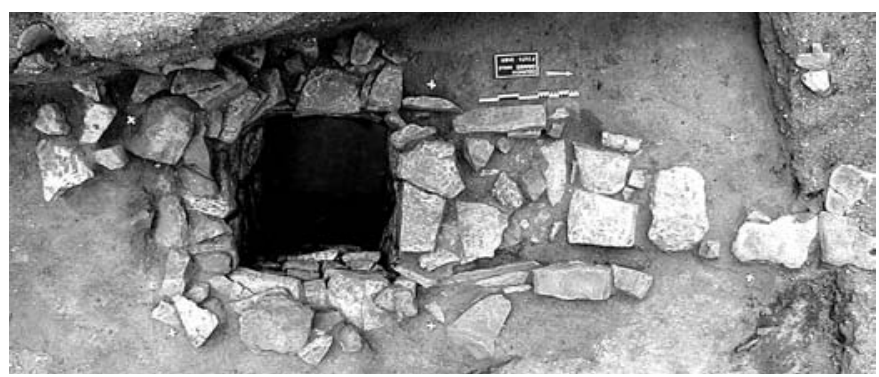

Fig. 25 - Le puits F2485, comblé à La Tène D2a, mis au jour dans le bassin de Sarliève (cliché : G. Vernet, INRAP Clermont-Ferrand).

pl. II, hors texte] reconnu plus à l'est dans les années 1980 à l'occasion de travaux de construction (Loison et al., 1991, p. 100). L'espace est fortement structuré par des palissades et des fossés qui forment des enclos coalescents. À l'intérieur de ces enclos, des alignements de trous de poteau révèlent l'emplacement de bâtiments. Plusieurs puits sont également à signaler, ainsi que des fosses dont la fonction finale a été de servir de dépotoir. Enfin, quatre structures ont livré des déchets de tabletterie montrant la présence d'au moins deux ateliers durant La Tène D. Les artisans ont fabriqué exclusivement des «rondelles en os ». Des ossements de bœuf, de cheval, de Cervus elaphus et d'Homo ont été utilisés pour la réalisation de ces rondelles (étude inédite de P. Caillat).

\section{Étude archéologique et paléopathologique de la nécropole}

Une trentaine de sépultures ont été mises au jour sur l'ensemble du site (fig. 24). Une vingtaine, qui peuvent être regroupées, forment une nécropole [CO-083, fig. 18 et pl. II, hors texte] d'au moins $500 \mathrm{~m}^{2}$. L'analyse des dépôts de mobilier céramique (31 vases) permet de proposer un champ chronologique relativement restreint pour la mise en place de ces inhumations : vers 140-90 av. J.-C. (La Tène D1a). Elle montre l'importance accordée à la nourriture (boissons et mets solides) dans le rituel funéraire. Les offrandes alimentaires (représentées par la vaisselle céramique et les dépôts de faune) occupent, en effet, une place privilégiée à côté des objets de parure (Y. Deberge, inédit).

D’après l'étude paléopathologique réalisée sur les squelettes (M. Billard, inédit), près d'un tiers des individus présentent les traces d'une hyperostose porotique du crâne, en particulier, ici, du plafond orbitaire (criba orbitalia). Les possibles pathologies responsables sont des anémies hémolytiques génétiques, des hémoglobinoses (thalassémie et drépanocytose), des affections parasitaires entraînant une 
perte sanguine par hémolyse (paludisme), ou une consommation de globules rouges (ankylostomiase) ou de vitamine B12 provoquant une érythropoièse inefficace au transport d'oxygène (diphyllobothtrium, parasite de certains poissons). Le rôle des phytates alimentaires (céréales) vient renforcer cette carence d'apport.

L'hyperostose porotique apparaît donc dans un complexe multi-factoriel de « misère alimentaire " (carence en fer, fuites intestinales parasitaires, carence protéique, rachitisme, malnutrition maternelle durant la grossesse, naissance prématurée). Il faut noter aussi la présence d'atteintes tuberculeuses, et peut-être brucelliennes, qui attesteraient une contamination par contact avec le bétail ou par consommation du lait. Ces informations doivent être discutées à la lumière des données archéologiques et paléoenvironnementales. On notera déjà que la proximité entre l'habitat et des zones palustres ou lacustres peut être un facteur important pour certaines des pathologies responsables de l'hyperostose porotique.

\section{L'OCCUPATION DU SITE}

\section{LORS DE LA CONQUÊTE ROMAINE}

Plusieurs structures présentes sur le site montrent que ce secteur était occupé au moment de la bataille de Gergovie. Par exemple, on citera le puits F2485 (fig. 25

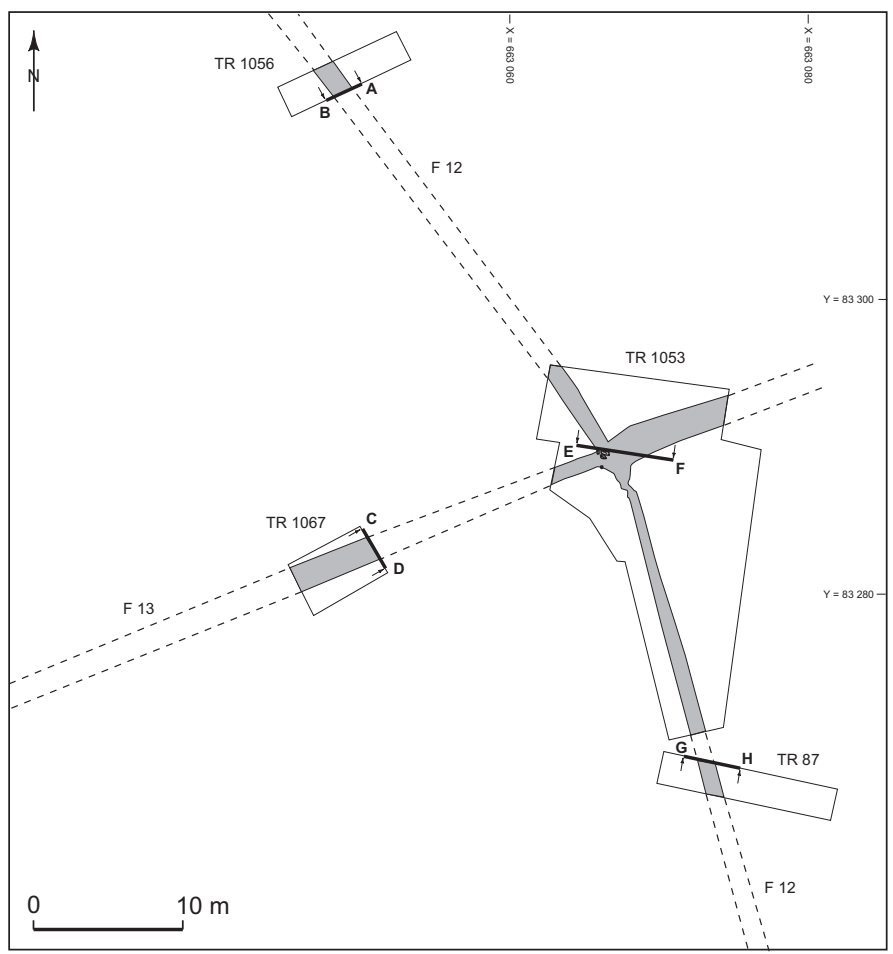

et pl. II, hors texte), qui a livré un lot de céramique très homogène, dont le dépôt peut être situé entre 80-70 et 50 av. J.-C. (La Tène D2a). Un autre puits (F2249/2487, pl. II, hors texte), double, a fourni un lot de céramique caractéris-tique de la fourchette chronologique comprise entre 50 et 20 av. J.-C. (A. Wittmann, inédit). Ce même puits a livré une monnaie identifiée comme un bronze frappé à la légende DONNADV, attribuée aux Arvernes. Enfin, il faut ici faire mention du parcellaire borné, mis en évidence au centre de la cuvette, qui peut être daté au plus tard du début de la période augustéenne (fig. 26). À cette époque, il est donc certain que le lac était très réduit, voire inexistant.

\section{L'OCCUPATION GALLO-ROMAINE}

Le site reste occupé après la conquête, avec la mise en place de plusieurs bâtiments construits en dur. L'un d'eux [CO-084, fig. 20 et pl. II, hors texte], daté du I ${ }^{\mathrm{er}}$ s. de notre ère (A. Wittmann, inédit), a été complètement dégagé. De plan rectangulaire et d'orientation nord-sud, il mesure environ $20 \mathrm{~m}$ x $13 \mathrm{~m}$ et est constitué d'une galerie de façade bordée de deux petites pièces, en avant d'un ensemble de cinq ou six pièces d'une superficie totale de $150 \mathrm{~m}^{2}$. À une trentaine de mètres à l'est, un second bâtiment [CO-085] de plan quadrangulaire a été très partiellement dégagé, sur
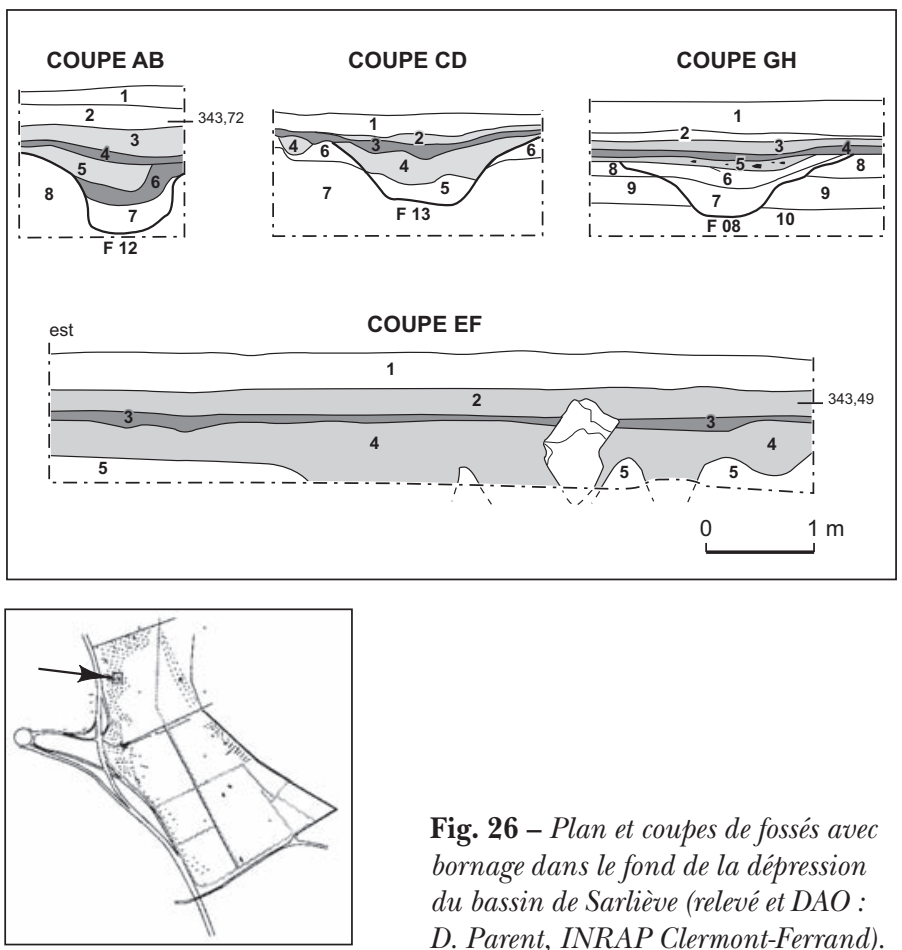

Fig. 26 - Plan et coupes de fossés avec bornage dans le fond de la dépression du bassin de Sarliève (relevé et DAO : D. Parent, INRAP Clermont-Ferrand). 
une superficie d'environ $11 \mathrm{~m}$ x $6 \mathrm{~m}$. Décentré vers le sud par rapport au premier bâtiment, il comprend au moins deux pièces. Ces deux édifices, orientés selon la même direction, forment un ensemble cohérent, délimité au sud par un mur de clôture repéré sur près de $35 \mathrm{~m}$ de long, qui devait former un angle droit avec le mur contre lequel s'appuie le bâtiment à galerie de façade.

Dans la partie sud de la zone fouillée, à plus de $150 \mathrm{~m}$ de ce premier ensemble, un bâtiment rectangulaire [CO-086] d'environ $9 \mathrm{~m}$ x $4 \mathrm{~m}$ a été mis au jour. Délimité lui aussi, au moins au sud et à l'ouest, par un mur de clôture, il se compose d'au moins deux pièces possédant des enduits peints et un sol en mortier hydraulique. Dans la pièce orientale, un petit bassin maçonné d'environ 0,80 m x 0,50 m était en relation directe avec un captage de source, également maçonné. Un système de goulottes en bois parfaitement conservées permettait l'évacuation de l'eau. Il semble que cet ensemble ait fonctionné jusqu'au III $^{\mathrm{e}} \mathrm{s}$.

Le vestige antique le plus récent ( $\mathrm{III}^{\mathrm{e}}-\mathrm{IV}^{\mathrm{e}} \mathrm{s}$. ?) est une canalisation d'eau potable constituée d'éléments préfabriqués en terre cuite, qui traverse la zone étudiée du sud-est vers le nord-ouest.

\section{LES PÊCHERIES MÉDIÉVALES ET MODERNES}

Enfin, le décapage a permis de reconnaître un ensemble de fossés et d'alignements de trous de poteau qui se développent parallèlement au paléorivage (pl. II, hors texte). Ces structures, comblées par des sédiments lacustres, peuvent être rapportées aux pêcheries mentionnées par les textes sur les bords du lac durant tout le Moyen Âge et jusqu'au $\mathrm{XVII}^{\mathrm{e}} \mathrm{s}$.

G. V.

\section{CONTEXTE GÉOLOGIQUE ET ÉVOLUTION DE LA SÉDIMENTATION DANS LE MARAIS DE SARLIÈVE À L'HOLOCÈNE}

\section{LA FORMATION DU LAC DE SARLIÈVE}

L'origine et le fonctionnement lacustre des dépressions semi-closes de Limagne (marais de Ravel, Seychalles, Sarliève) ont intéressé de nombreux auteurs, parmi lesquels M. Derruau (1949), L. Gachon (1963), S. B. Kroonenberg et al. (1987) et G. Lenselink et al. (1990). Les avis divergent quant à l'origine de la dépression de Sarliève: blocage alluvial par le cône de l'Artière (Derruau, 1949), creusement puis obturation des vallées par colluvionnement permettant la mise en eau du lac (Gachon, 1963 ; Lenselik et al., 1990).

Les forages réalisés par le laboratoire de Géologie des environnements aquatiques continentaux (GéEAC) dans la zone d'exutoire septentrionale du marais et immédiatement au nord ont montré, en plus d'une remontée du substratum, un dépôt superficiel très hétérométrique, à matrice fine emballant du sable et des graviers de calcaire oligocène (fig. 6). Ce faciès traduit des coulées boueuses, vraisemblablement issues d'un vallon latéral situé à l'est, dont la tête actuellement comblée s'initiait au niveau du puy Long. En outre, les cotes altimétriques actuelles révèlent un seuil dans le substrat (toit à une cote de 347 m à 349 m NGF) à la sortie aval de la dépression dont le fond se trouve à la cote 338,50 m NGF : il s'agit d'une cuvette fermée, aux limites apparemment assez abruptes (fig. 27 et 28). On peut donc supposer que la dépression a une origine initiale néotectonique, liée au rejeu des grands accidents nord-sud de la Limagne, possible dans un contexte de volcanisme actif au Tardiglaciaire et au début de l'Holocène (Vernet, Raynal, 2000). L'effet de barrage a pu être renforcé par des coulées boueuses, entraînant la mise en eau ou l'approfondissement du lac. La position très superficielle des coulées recoupées par forage évoque un âge holocène, les coulées boueuses pouvant apparaître facilement en climat tempéré humide, dans un contexte marno-calcaire.

La première mise en eau de la dépression est un peu antérieure au téphra ${ }^{6} \mathrm{CF} 1$ dont la retombée, considérée comme directe et datée de 11750 cal. B.C. (Miallier et al., 2004), a recouvert une première unité de sédiments lacustres.

\section{ORIGINE DES SÉDIMENTS LACUSTRES ET ÉVOLUTION DE LA PRODUCTION DE MATIÈRE}

\section{DÉPÔTS DE DELTAS ET DÉPÔTS DE BASSINS}

Les observations faites dans les sédiments du marais ont permis d'identifier deux principaux types de remplissage (fig. 27 à 29) : les dépôts de deltas (SP4) et les dépôts de bassins (SARL 10). Les dépôts de deltas, mis en évidence dans la partie médiane plus étroite du marais, sont caractérisés par la présence de deux épais deltas sableux silico-

6. Retombée volcanique. 


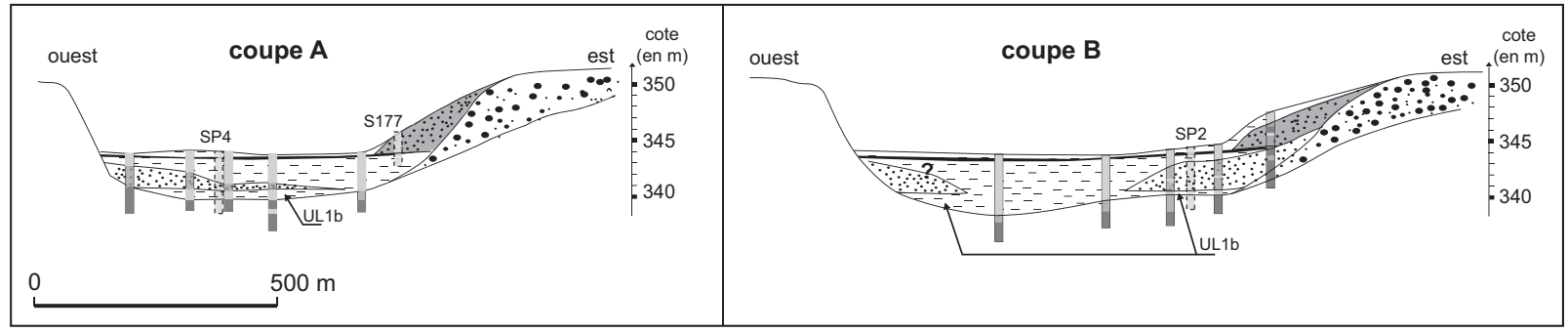

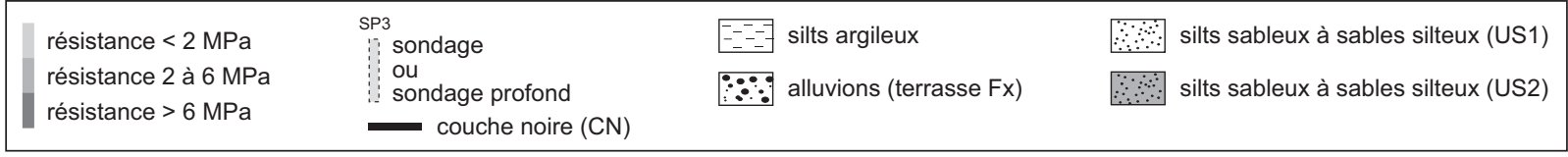

Fig. 27 - Coupes synthétiques ouest-est basées sur les données des tests de pénétrométrie statique calibrés d'après des sondages profonds réalisés dans le bassin de Sarliève (localisation des traits de coupes, voir fig. 6 et 7) : S, sondage; SP, sondage profond; UL, unité lacustre (DAO : J.-G. Bréhéret, A. Fourmont et J.-J. Macaire).

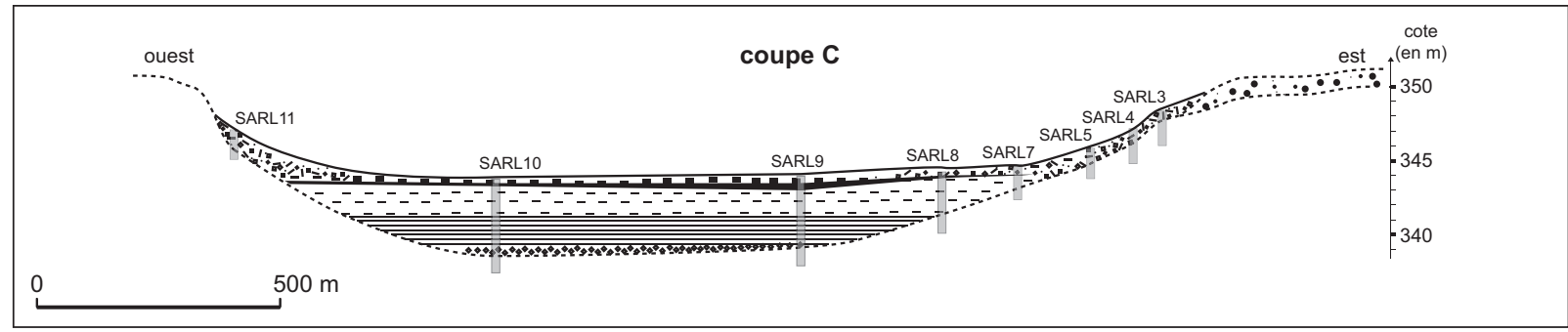

\begin{tabular}{|c|c|c|c|}
\hline sondage & Colluvions & -:- silts argileux verdâtres (UL4) & 产 laminites (UL2) \\
\hline couche noire $(\mathrm{CN})$ & $\because \because$ alluvions (terrasse Fx) & ${ }_{--}^{--}$silts argileux verdâtres (UL3) & silts argileux gris bleuté (UL1a) \\
\hline
\end{tabular}

Fig. 28 - Coupe synthétique ouest-est du bassin sud de Sarliève réalisée à partir de forages carottés (SARL). Localisation du trait de coupe, voir fig. 6 (DAO :J.-G. Bréhéret, A. Fourmont et J.-J. Macaire).
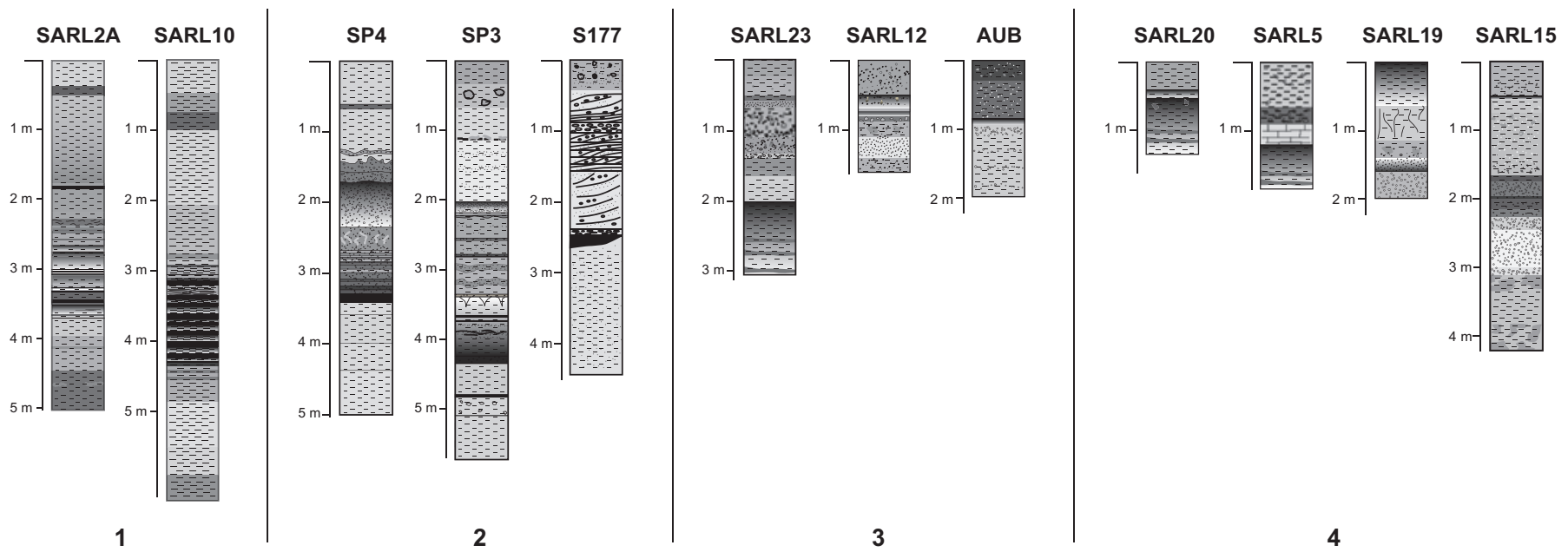

Fig. 29 - Représentation des logs sédimentaires du bassin de Sarliève (carottes, sondages et sondages profonds) décrits dans le texte: 1, SARL $2 A$ et SARL 10 dans les bassins nord et sud du marais ; 2, SP 4, SP 3 et S 177 dans les zones de delta ouest et est du marais; 3, SARL 23, SARL 12 et AUB dans la zone d'exutoire à "coulées boueuses"; 4, SARL 20, SARL 5, SARL 19 et SARL 15 au niveau des bordures est et ouest du marais (DAO :J.-G. Bréhéret, A. Fourmont et J.-J. Macaire). 


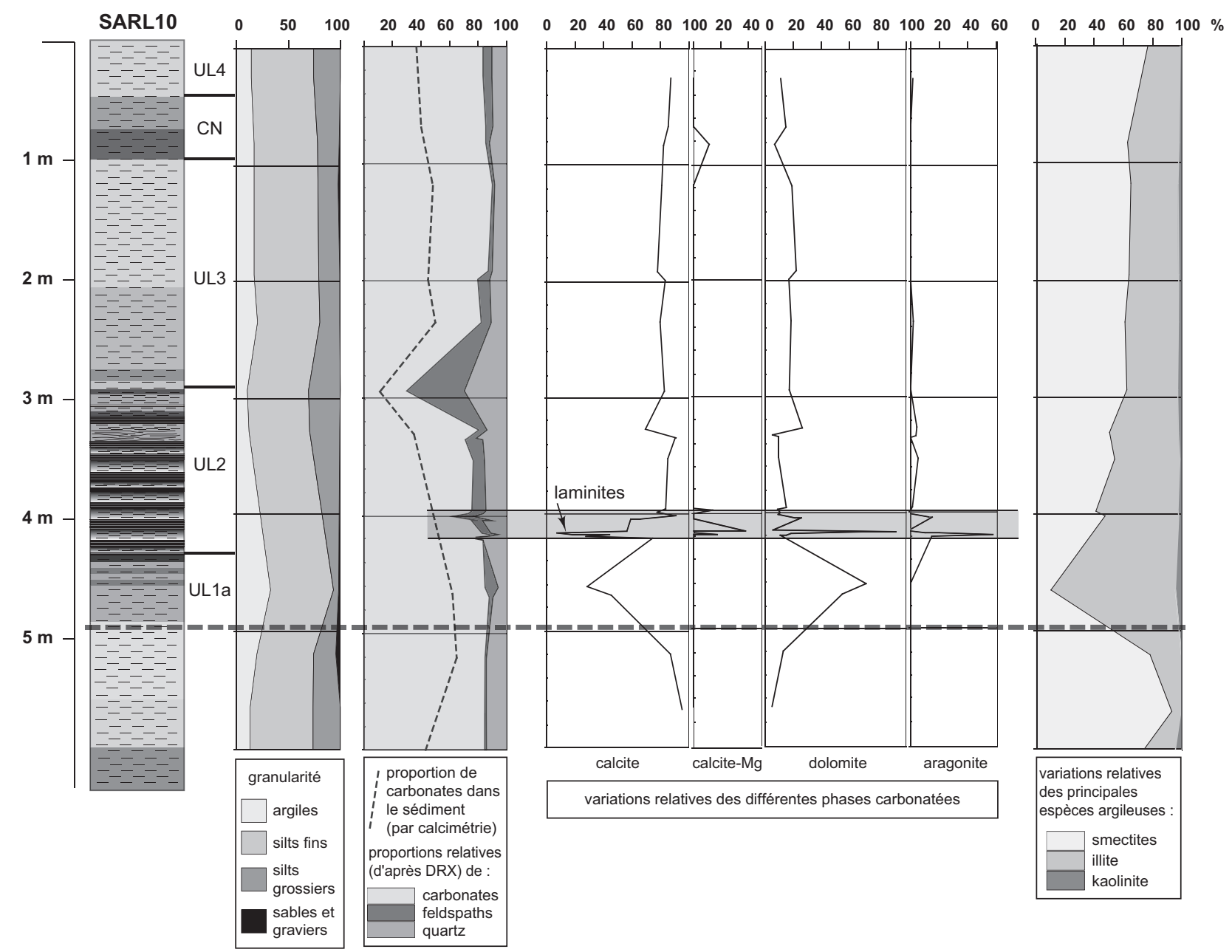

Fig. 30 - Minéralogie des sédiments du carottage SARL 10 (bassin sud de Sarliève). La ligne horizontale en pointillés matérialise le toit du substratum : UL, unité lacustre ; CN, couche noire (DAO : J.-G. Bréhéret, A. Fourmont et J.-J. Macaire).

clastiques qui s'intercalent dans des sédiments silto-argileux carbonatés : un delta ancien (US1) et un plus récent (US2), observé seulement au niveau de la bordure occidentale du marais (fig. 6, coupes A et B). Les dépôts de bassins, rencontrés dans les lobes nord et sud du marais, sont en revanche seulement constitués de sédiments silto-argileux carbonatés (fig. 6, coupe C). Les travaux du GéEAC ont permis de préciser la nature et l'origine des sédiments de ces deux principaux types de remplissage (fig. 30 à 33).

\section{ORIGINE DES SÉDIMENTS LACUSTRES}

L'érosion des versants peut être de deux types : l'érosion mécanique, à l'origine de la production solide de particules piégées en totalité ou en partie dans la cuvette (détritisme), et l'érosion chimique, à l'origine de la production dissoute susceptible d'être piégée en totalité ou en partie par précipitation chimique et/ou bioinduite in situ. La distinction de ces deux phases, "détritique » ou "précipitée », permet d'avoir des renseignements sur les processus d'érosion sur les versants, en liaison avec le climat et l'occupation du sol, ainsi que sur certaines caractéristiques hydrologiques (profondeur d'eau) et hydrochimiques (salinité) du lac. Cependant, la distinction entre ces deux types de matière (solide ou dissoute) est délicate, car les matériaux présents dans le bassin-versant (substrats et sols) sont en partie constitués de carbonates (calcite et dolomite en proportions variables), que l'on trouve aussi dans les sédiments lacustres, où ils peuvent, soit avoir une origine détritique, soit résulter d'une précipitation. Les sédiments lacustres comportent aussi des silicates (quartz, feldspaths, minéraux argileux de type smectites et illite), mais ceux-ci sont essen- 


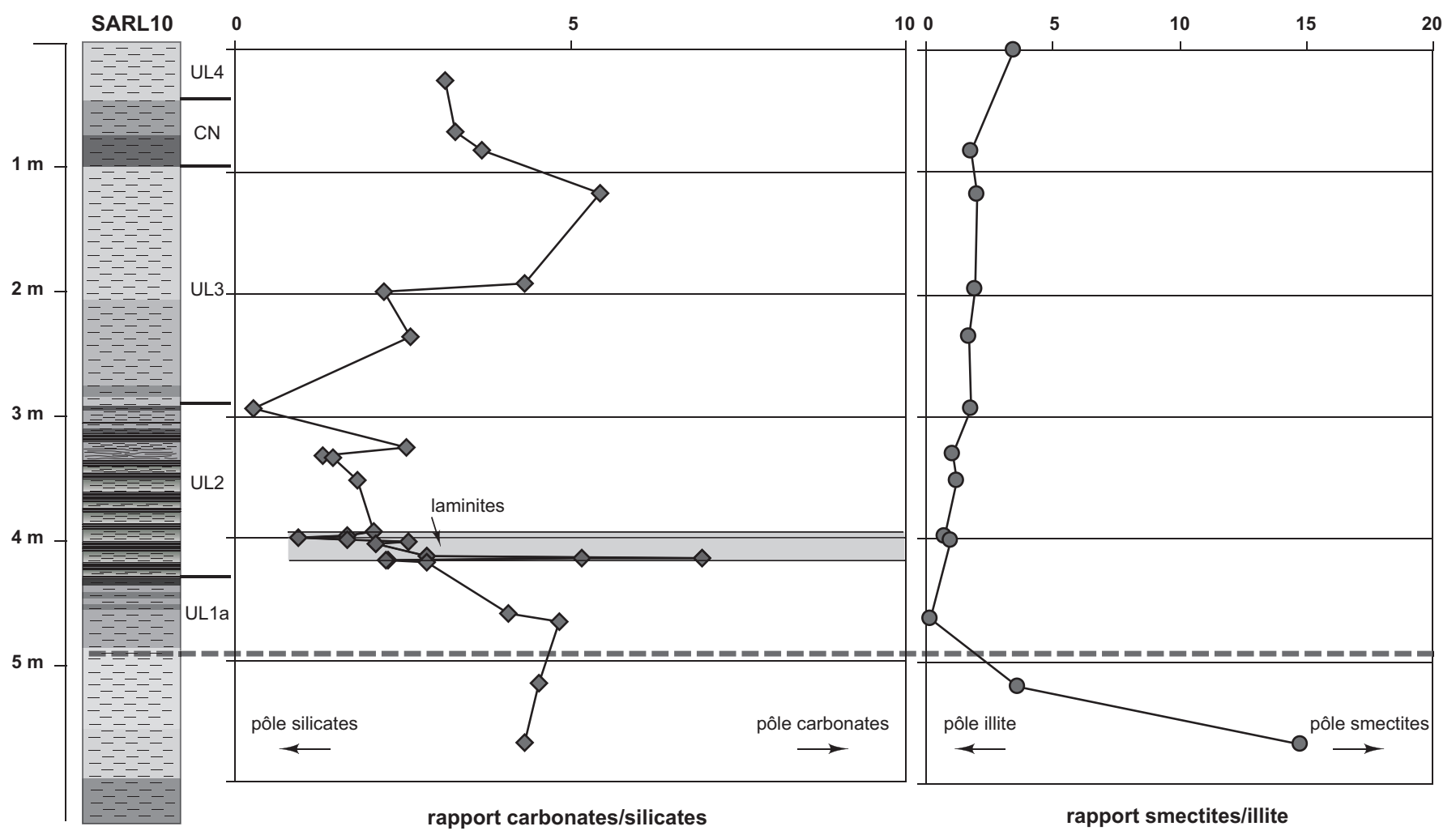

Fig. 31 - Variations des rapports carbonates/silicates et smectites/illite provenant du carottage SARL 10 réalisé dans le bassin de Sarliève. La ligne horizontale en pointillés matérialise le toit du substratum: UL, unité lacustre ; CN, couche noire (DAO : J.-G. Bréhéret, A. Fourmont et J.J. Macaire).

tiellement issus de l'érosion mécanique des versants. Les observations au microscope électronique à balayage ont permis de préciser l'origine des carbonates des sédiments lacustres (Fourmont, 2005).

\section{Sédiments de bassins}

La dolomite qui compose l'unité lacustre UL1a se présente sous forme de cristaux de taille nano- à micrométrique dont l'habitus suggère une formation in situ (fig. 30). Il en est de même de la dolomite que l'on trouve dans les lamines et macules de UL2. La précipitation de dolomite témoigne d'apports dissous dominant largement le détritisme, associés à des conditions particulières dans la dépression lacustre, en l'occurrence une faible tranche d'eau saumâtre dans un bassin fermé. Les silts argileux qu'encadrent les lamines carbonatées dans UL2 semblent comporter de la calcite détritique et précipitée, et de la dolomite clairement détritique; son habitus est identique à celui observé dans les marno-calcaires oligocènes : rhomboèdres de la taille des silts (Bréhéret et al., 2003).
Les sédiments des unités lacustres UL3 et UL4 et ceux de la couche noire (CN) ont une composition minéralogique homogène avec, à l'instar des sédiments «interlamines » décrits précédemment, calcite détritique et précipitée et dolomite détritique. Des indices minéraux de l'érosion mécanique apparaissent dans l'unité laminée et se pérennisent dès UL3. Notons que la couche noire est riche en restes de pyrofusinites formés par la combustion de végétaux terrestres (Tyson, 1995), témoin de forts brûlis sur les versants.

Enfin, la proportion de smectites augmente légèrement de la base au sommet de la série au détriment de l'illite (fig. 31). Cette évolution pourrait refléter une tendance à la dégradation de l'illite et sa transformation en smectites au cours de l'Holocène, au fur et à mesure du développement des sols.

\section{Sédiments de zones de deltas}

Dans l'unité UL1b, la majorité des carbonates semble détritique (fig. 32). Le delta US1 est dominé par des 


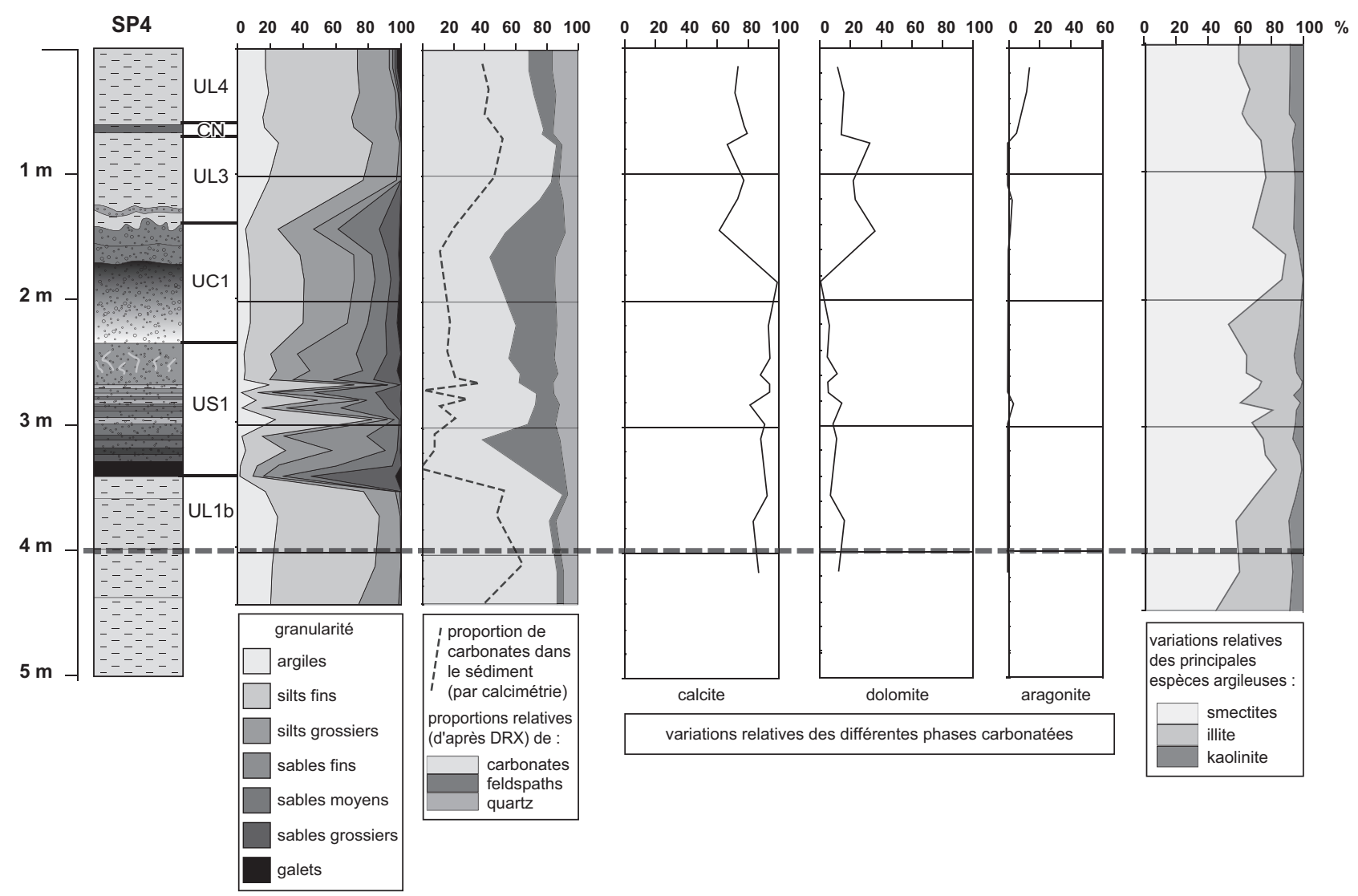

Fig. 32 - Minéralogie des sédiments provenant du sondage profond SP4 réalisé dans le bassin de Sarliève (delta ouest). La ligne horizontale en pointillés matérialise le toit du substratum: UL, unité lacustre; CN, couche noire; UC, unité colluviale; US1, delta ancien (DAO : J.-G. Bréhéret, A. Fourmont et J.-J. Macaire).

apports détritiques silico-clastiques, avec une petite proportion de calcite, probablement issue de l'érosion du substrat calcaire. L'unité colluviale UC1 a également été alimentée par des apports détritiques. Les unités UL3, UL4 et $\mathrm{CN}$ des dépôts de deltas ont une composition similaire à celle des mêmes unités de bassins, avec toutefois un peu plus de dolomite détritique. Les phases détritiques sont plus abondantes et nettes dans la zone de deltas située au débouché de thalwegs, où les eaux concentrées ont une plus grande énergie, et sont peu ou pas perceptibles dans les bassins.

Enfin, les smectites dominent l'illite dans l'ensemble de la séquence avec toutefois une légère augmentation de l'illite dans le delta ancien US1 jusqu'à la base des colluvions UC1, peut-être liée à un ralentissement de la pédogenèse et à l'altération de la couverture végétale (fig. 33). On observe en outre un pic de smectites dans la partie supérieure de UC1, probablement en relation avec une paléopédogenèse.

\section{SCHÉMA DE L'ÉVOLUTION DU GÉOSYSTÈME LACUSTRE}

Suite à la fermeture du système, probablement durant le Bölling, des sédiments lacustres se sont mis en place par précipitation in situ ou par détritisme. Le schéma évolutif peut être décrit en deux phases successives (fig. 34 et tabl. I).

\section{PHASE 1 : BÖlling-ATlantiQUe}

\section{Zones de deltas}

Dans les zones de deltas, les premiers sédiments (UL1b) se sont déposés avant 11750 cal. B.C., âge attribué au téphra CF1 qui les recouvre (Vernet, Raynal, 2000). La retombée, quasi instantanée, de CF1 est suivie par la mise en place, probablement rapide, du delta sableux (US1) qui remanie le téphra ayant saupoudré les versants. Ces dépôts deltaïques à dominante d'apports détritiques témoignent d'une forte 


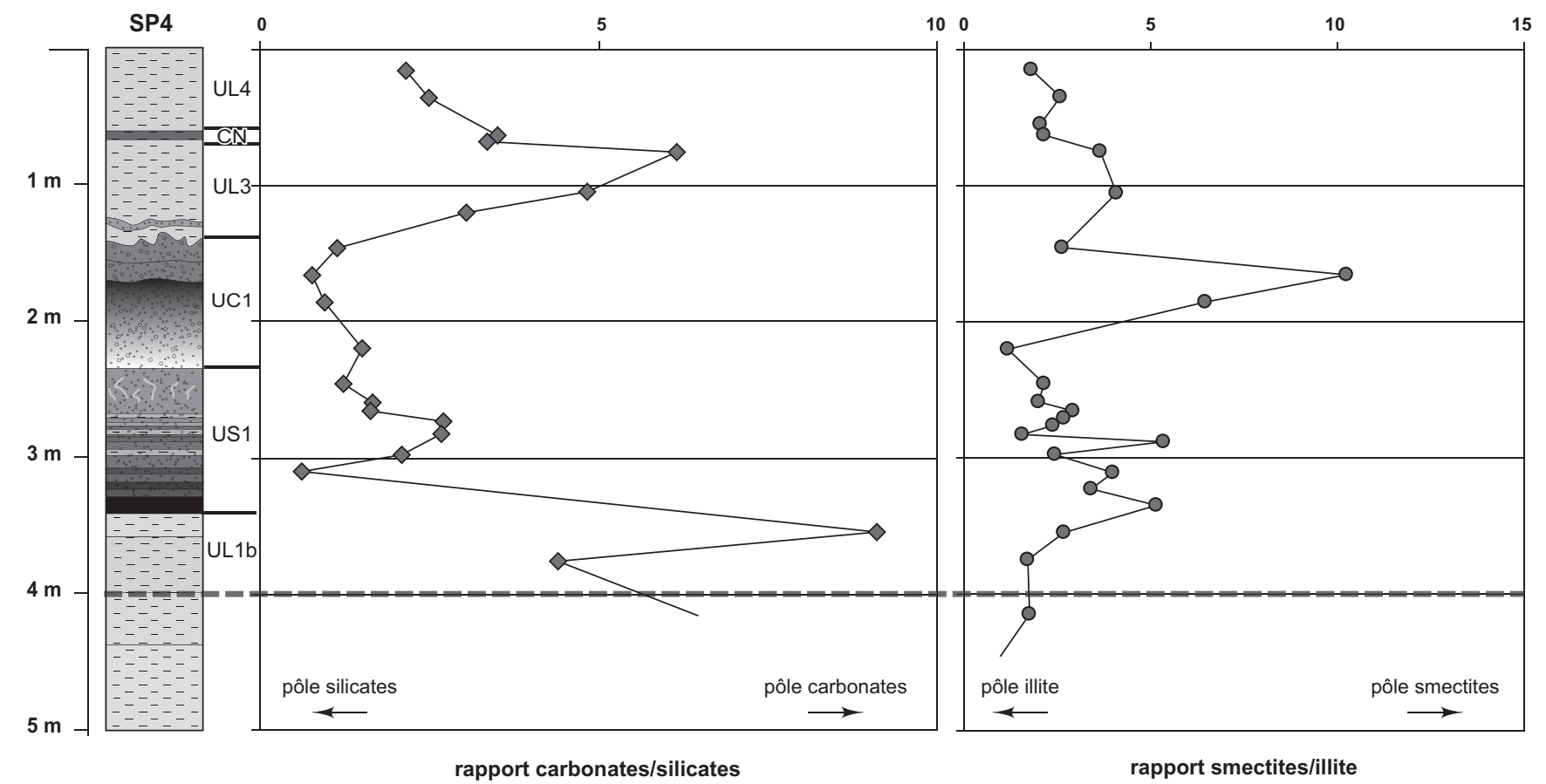

Fig. 33 - Variations des rapports carbonates/silicates et smectites/illite provenant du sondage profond SP4 réalisé dans le bassin de Sarliève (les strates constituées uniquement de sables n'ont pas fait l'objet d'analyses par diffraction des rayons X (DRX), et ne figurent donc pas dans le rapport carbonates/ silicates). La ligne horizontale en pointillés matérialise le toit du substratum: UL, unité lacustre; CN, couche noire; UC, unité colluviale; US1, delta ancien (DAO :J.-G. Bréhéret, A. Fourmont et J.J. Macaire).

production solide, peut-être de façon temporaire, durant l'Alleröd et/ou le Dryas récent. Le delta US1, dont la base, soulignée par le téphra CF1 considéré en place, se situe à $341 \mathrm{~m} \mathrm{NGF}$, s'est obligatoirement mis en place sous une tranche d'eau dont la cote a dépassé le toit de l'unité, soit environ 342 m NGF (fig. 27 et 28).

Avant environ 9550 cal. B.C., probablement au cours du Dryas récent, l'accrétion sédimentaire et sans doute la diminution du niveau d'eau ont amené l'émersion des zones de deltas. Dès le début de l'Holocène, cette émersion a été suivie d'une phase de colluvionnement qui a pu être assez brève. Ces colluvions UC1 comportent une forte composante silto-sableuse (quartz et feldspaths) et une fraction carbonatée réduite. L'enrichissement en smectites et la diminution de la calcite dans la partie supérieure de cette formation confirment la présence du paléosol montré par le faciès. Colluvionnement et pédogenèse impliquent l'émersion durable des zones deltaïques durant le Préboréal, le Boréal et l'Atlantique, avant une nouvelle et importante remontée du niveau de l'eau (phase 2). Le toit des colluvions pédogénisées (UC1) se situant à une cote d'environ 343-344 m NGF, le niveau de l'eau était plus bas durant toute cette période.

\section{Zones de bassins}

Dans les zones de bassins, les premiers sédiments lacustres mis en place sont très fins et très carbonatés (unité lacustre UL1a), riches en dolomite, dont la formation in situ a nécessité une eau saumâtre peu profonde, en contexte endoréïque, avec peut-être des alternances de mise en eau et d'assèchement. Deux plans d'eau d'extension limitée (jusqu'à une cote d'environ 339 m NGF) ont pu être isolés au moins temporairement au fond des deux bassins, l'un au nord, l'autre au sud. Ainsi, jusqu'à environ 5850 cal. B.C., date du dépôt des sédiments de UL2, les sédiments déposés dans les bassins témoignent d'un haut degré de confinement et d'une production solide limitée sur les versants. Dès lors, les sédiments de l'unité UL2 ont commencé à se déposer en une alternance de couches interlamines à composante détritique et de faisceaux de lamines constituées de carbonates précipités. Ces derniers correspondraient à des épisodes, répétés au cours du dépôt de UL2 jusqu'à la fin de l'Atlantique, de diminution de l'érosion mécanique associée à une diminution de la tranche d'eau et à un enrichissement en solutés. 


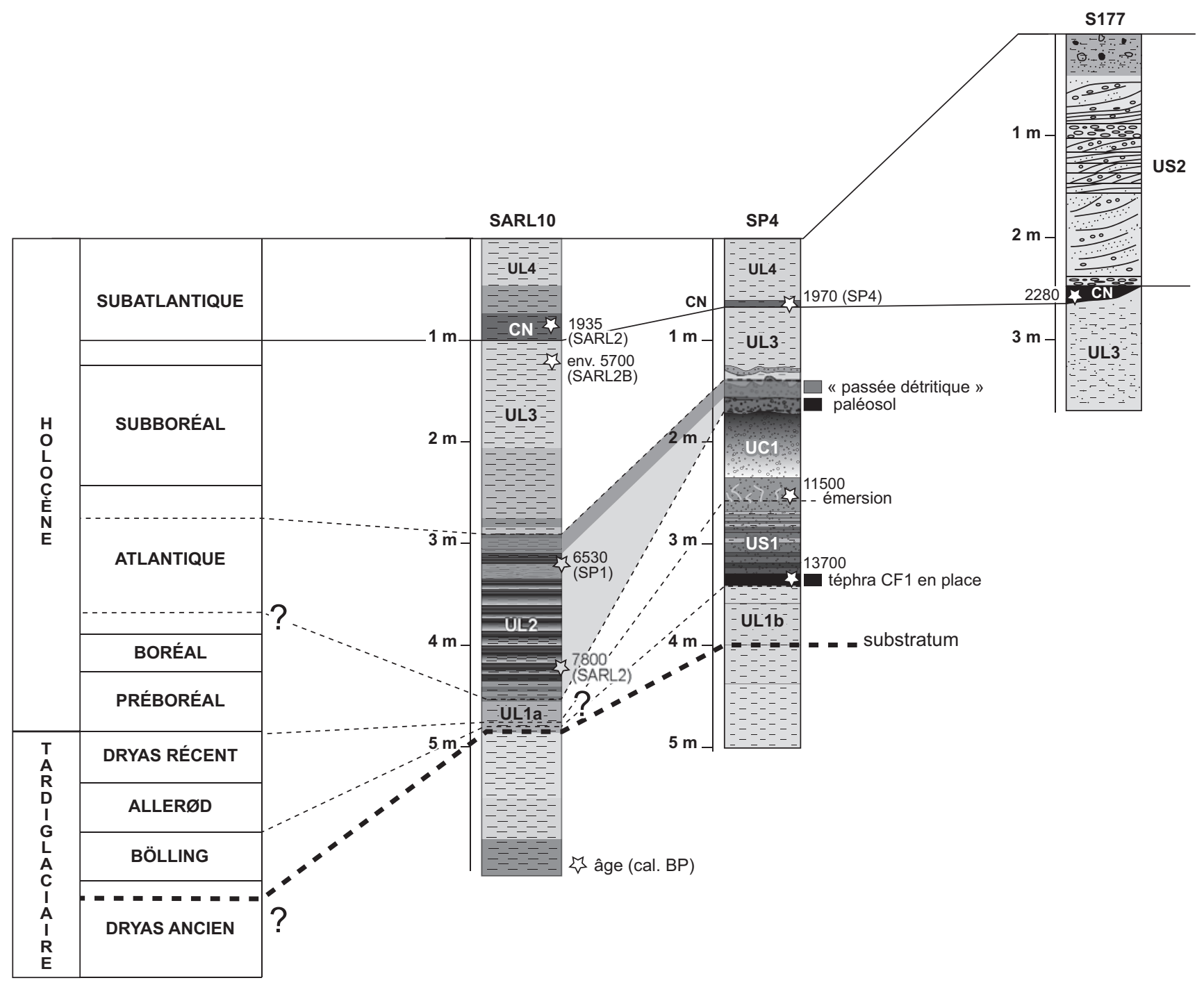

Fig. 34 - Corrélations chronostratigraphiques entre les sédiments de bassins et les sédiments de deltas du lac de Sarliève :

UL, unité lacustre; CN, couche noire ; UC, unité colluviale ; US1, delta ancien ; US2, delta récent (DAO :J.-G. Bréhéret, A. Fourmont et J.-J. Macaire).

Au cours de cette première phase, le synchronisme entre les dépôts de deltas et ceux des bassins n'est pas encore clair. En effet, malgré les nombreux forages, le téphra CF1, net dans le delta, n’apparaît pas dans les bassins. D’autre part, les épais dépôts deltaïques (US1) n’ont pas d'équivalents nets, déposés en eau profonde dans les bassins, à la base des séquences : c'est le cas notamment de UL1a, très riche en dolomite formée in situ, dont la mise en place sous une faible tranche d'eau saumâtre à salée est peu compatible avec l'épisode deltaïque. Il est toutefois possible de mettre en relation l'unité UL2 laminée des bassins (commençant à l'Atlantique inférieur/moyen) avec les colluvions pédo- génisées UC1 du delta, ces deux faciès marquant un niveau d'eau plutôt bas. Des datations ${ }^{14} \mathrm{C}$ et des analyses minéralogiques complémentaires sont attendues pour éclaircir ce problème, notamment la position des chronozones de la fin du Tardiglaciaire et du début de l'Holocène dans les dépôts de bassin.

\section{PHASE 2 : SUbBORÉAL-ACTUEL}

Après cette première phase, le niveau de l'eau s'est élevé vers la fin de l'Atlantique ou le début du Subboréal, entraînant le dépôt de l'unité lacustre UL3 sur l'ensemble 
Tabl. I - Datations ${ }^{14} C$ des sédiments du marais de Sarliève.

\begin{tabular}{|c|c|c|c|c|c|c|}
\hline $\mathbf{N}^{\circ}$ d'échantillon & \begin{tabular}{|c|} 
Unité \\
lithologique
\end{tabular} & Nature de l'échantillon & Code labo. Lyon & Âge ${ }^{14} \mathrm{C}$ BP & Âge cal. BP & $\begin{array}{l}\text { Âge moyen } \\
\text { cal. BC/AD }\end{array}$ \\
\hline SP1 60-65 & $\mathrm{CN}$ & MO charbonneuse dans sédiment & LY-11357 & $1885 \pm 30$ & 1882 à 1730 & $150 \mathrm{cal} . \mathrm{AD}$ \\
\hline SP1 65-70 & $\mathrm{CN}$ & MO charbonneuse dans sédiment & LY-11358 & $2030 \pm 30$ & 2044 à 1899 & 20 cal. BC \\
\hline SP1 274-276 & UL2 & MO dans sédiment & LY-11359 & $5740 \pm 40$ & 6657 à 6412 & 4590 cal. BC \\
\hline SP1 298-300 & UL2 & MO dans sédiment & LY-11360 & $6055 \pm 35$ & 6997 à 6762 & 4930 cal. BC \\
\hline SP1 320 & UL2 & MO dans sédiment & LY-11361 & $6045 \pm 40$ & 6998 à 6757 & 4930 cal. BC \\
\hline SP4 65-69 & $\mathrm{CN}$ & MO charbonneuse dans sédiment & Lyon-2002 (GrA-22118) & $1940 \pm 60$ & 1999 à 1728 & $90 \mathrm{cal} . \mathrm{AD}$ \\
\hline SP4 235-250 & UC1 & racines dans sédiment & LY-11362 & $9985 \pm 65$ & 11698 à 11229 & 9510 cal. BC \\
\hline S28 82-85 & CN remaniée & MO charbonneuse dans sédiment & LY-11363 & $1465 \pm 35$ & 1411 à 1295 & 600 cal. AD \\
\hline S177 260-270 & $\mathrm{CN}$ & MO charbonneuse dans sédiment & LY-11364 & $2310 \pm 35$ & 2353 à 2212 & 330 cal. BC \\
\hline SARL2A 40-45 & $\mathrm{CN}$ & MO charbonneuse dans sédiment & LY-9813 & $1935 \pm 35$ & 1999 à 1869 & 20 cal. AD \\
\hline SARL2A 333-336 & UL2 & MO dans sédiment & LYON-1236 (OXA) & $6945 \pm 65$ & 7934 à 7666 & 5850 cal. BC \\
\hline SARL2B 120 & UL3 & MO dans sédiment & Lyon-1985 (GrA-21830) & $4930 \pm 70$ & 5887 à 5492 & 3740 cal. BC \\
\hline SARL2B 205 & UL2 & MO dans sédiment & Lyon-1984 (GrA-21829) & $6480 \pm 70$ & 7556 à 7267 & 5460 cal. BC \\
\hline SARL2B 231 & UL2 & MO dans sédiment & Lyon-1983 (GrA-21827) & $5770 \pm 70$ & 6728 à 6408 & 4620 cal. BC \\
\hline SARL2B 260 & UL2 & MO dans sédiment & Lyon-1982 (GrA-22023) & $6390 \pm 50$ & 7424 à 7248 & 5390 cal. BC \\
\hline SARL2B 384 & UL1a-UL2 & graines & Lyon-1981 (GrA-21826) & $6890 \pm 80$ & 7925 à 7587 & 5810 cal. BC \\
\hline SARL1 233-235 & UL2 & MO dans sédiment & LYON-1235 (OXA) & $6050 \pm 50$ & 7138 à 6754 & 5000 cal. BC \\
\hline SARL1 410-428 & UL1a-UL2 & MO dans sédiment & LY-9812 & $6720 \pm 55$ & 7670 à 7484 & 5630 cal. BC \\
\hline SARL0 235 & UL2 & MO dans sédiment & LY-775 (OxAA) & $6625 \pm 70$ & 7557 à 7345 & 5500 cal. BC \\
\hline
\end{tabular}

du marais. Les sédiments de UL3 semblent être le produit d'une érosion mécanique dominante sur les versants, avec une augmentation de l'apport en particules carbonatées, probablement liée à un amincissement des sols. Les silts argileux de UL3 paraissent résulter d'une transgression qui se traduit par une plus grande profondeur d'eau et une plus grande extension lacustre; les sédiments couvrent l'ensemble du marais jusqu'à une cote d'environ 343 m NGF, niveau de la couche noire qui recouvre ces sédiments sur l'ensemble de la surface du marais. La limite inférieure de cette couche (avec UL3) est souvent plus nette que la limite supérieure, plus diffuse. La couche noire, que les datations ${ }^{14} \mathrm{C}$ placent toujours dans le Subatlantique (entre 403-262 cal. B.C. et 68-220 cal. A.D.), pourrait s'être mise en place après une phase d'assèchement ou de très bas niveau lacustre au Subboréal ou au début du Subatlantique. Sa forte proportion de pyrofusinites témoigne de l'érosion mécanique des sols où le brûlis était pratiqué. Son dépôt serait synchrone d'une augmentation de la tranche d'eau qui s'est prolongée durant le Subatlantique avec la mise en place de UL4 jusqu'à l'assèchement du marais au XVII ${ }^{\mathrm{e}} \mathrm{s}$. Cette dernière unité lacustre, dont l'extension atteint une cote voisine de 344 m NGF (fig. 27 et 28), est caractérisée par une augmentation de la fraction détritique silicatée sur l'ensemble du marais. Ce dernier épisode correspond au plus haut niveau lacustre enregistré dans le marais : la cote du niveau de l'eau, donnée par celle du toit des sédiments du delta historique formé en bordure orientale du marais (delta US2), a dépassé 346,50 m NGF, inondant ainsi une partie des rives du lac. Par ailleurs, ce delta témoigne d'événements pluviométriques très violents qui ont pu affecter le système au cours de la période historique.

A. F., J.-J. M. et J.-G. B.

\section{LES DYNAMIQUES DE LA VÉGÉTATION DU NÉOLITHIQUE AU MOYEN ÂGE}

\section{ANALYSE POLLINIQUE ET MICROFOSSILES NON POLLINIQUES}

\section{LES DONNÉES PALÉOBOTANIQUES}

Les analyses paléobotaniques ont porté sur les sédiments lacustres et sur ceux des structures archéologiques. 


\section{Les dépôts lacustres}

Les figures 35 à 37 présentent les résultats obtenus pour le sondage SARL 2B (pollens et microfossiles non polliniques) et le sondage profond SP4 (pollens). Au total, 72 taxons polliniques différents ont été observés. La concentration pollinique est élevée (de l'ordre de 20000 grains/g de sédiment), sauf près de la surface, entre $14 \mathrm{~cm}$ et $60 \mathrm{~cm}$ de profondeur. Il en est de même pour les microfossiles non polliniques (35 taxons).

La somme pollinique totale servant au calcul des pourcentages de chaque taxon comprend tous les grains de pollen d'arbres et d'herbacées, ainsi que les spores, à l'exclusion des grains de pollen tertiaires issus des marnes oligocènes, voire miocènes, du substrat. Les variations des pourcentages de ces derniers, calculés par rapport à la somme pollinique totale, sont figurées par une courbe à droite du diagramme pollinique. L'abondance des grains de pollen tertiaires - souvent de Gymnospermes, bien reconnaissables car fortement usés et déformés - est considérée comme un bon indicateur de l'importance des phénomènes érosifs affectant les pentes des reliefs avoisinants.

\section{Biozones, chronologie pollinique et datations ${ }^{14} C$}

Le diagramme du carottage SARL 2B (fig. 35) peut être divisé en 10 zones polliniques locales, notées, de la plus ancienne à la plus récente, SARL 2B.a à SARL 2B.j (tabl. II). La même zonation a été retenue pour les microfossiles non polliniques. Les nombreuses données disponibles pour l'Holocène sur l'ensemble du Massif central permettent de replacer les assemblages polliniques de Sarliève dans l'évolution générale de la végétation (Beaulieu et al., 1988 ; Vergne, 1989 ; Reille, 1990 ; Miras et al., 2003) :

- la partie inférieure du diagramme (SARL 2B.a, b et c) est typique de la période atlantique. Les alentours du lac de Sarliève sont alors occupés par un riche milieu forestier (chênaie-corylaie à orme, tilleul et frêne). Ce milieu se modifie progressivement, avec d'abord la dispersion du hêtre puis du sapin à partir de leurs zones refuges (occurrences régulières), puis leur diffusion (SARL 2B.d), dès l'Atlantique final ;

- l'expansion de Fagus et celle plus limitée d'Abies, caractéristiques du Subboréal, s'effectuent ensuite (SARL 2B.e, $\mathrm{f}, \mathrm{g}, \mathrm{h})$;

- enfin, la régression brutale de tous les taxons forestiers (SARL 2B.i), puis leur quasi-disparition laissant place à un milieu très ouvert livré aux cultures (SARL 2B.j), typique du Subatlantique.
Afin d'étayer et de préciser la chronologie pollinique, une série de cinq datations ${ }^{14} \mathrm{C}$ réalisées par accélérateur (AMS) a été effectuée sur la colonne SARL 2B (tabl. I et II). La date des sédiments de la base du sondage $(384 \mathrm{~cm})$ a été obtenue sur des graines contemporaines du dépôt (Filipendula, Carex sp., taxons également représentés par leur pollen) recueillies par tamisage; les autres ont été réalisées à partir de la matière organique globale extraite des échantillons.

La date de la base, 5810 cal. B.C. $(384 \mathrm{~cm})$, ne pose pas de problème. Elle s'intègre parfaitement dans l'Atlantique moyen, bien reflété par les assemblages polliniques. En revanche, parmi ces cinq dates, le résultat relevé à $205 \mathrm{~cm}$ (5460 cal. B.C.) devrait être logiquement compris entre 4620 cal. B.C. $(231 \mathrm{~cm})$ et 3740 cal. B.C. $(120 \mathrm{~cm})$. Cette date trop ancienne ayant été rejetée, les quatre autres forment alors un ensemble cohérent, auquel on peut ajouter le contrôle de la date de la couche noire, obtenue dans le sondage proche SARL 2A (20 cal. A.D.), car elle constitue un repère sûr.

La diffusion du hêtre (fig. 35, zone d) commence à 5390 cal. B.C. Cette date paraît précoce par rapport à ce qui est admis pour le Massif central où, sur la base d'un corpus jugé cohérent de 22 dates, cet événement se situe vers 58005400 B.P., soit environ 4550-4050 cal. B.C. (Reille, 1990). De même, les dates 4620 cal. B.C. à la limite AtlantiqueSubboréal et 3740 cal. B.C. représentant l'optimum de la hêtraie semblent trop anciennes (d'environ 1000 ans) par rapport au cadre généralement admis. Ce décalage peut-il être imputé "à l'influence du $\mathrm{CO}_{2}$ d'origine volcanique ", comme le proposent A. Pons et al. (1988) pour les sites des régions du Massif central à volcanisme quaternaire (Velay et Cézallier) ? Ou à la présence dans les sédiments de résidus carbonés d'incendies passés mis en évidence par l'analyse des sédiments et des microfossiles non polliniques? Ces hypothèses peuvent être envisagées, mais, par ailleurs, il faut noter aussi que tous les sites de référence utilisés par les auteurs précédents se situent entre $975 \mathrm{~m}$ et $1400 \mathrm{~m}$ d'altitude, alors que le bassin de Sarliève n'est qu'à 345 m. Cette situation a dû jouer un rôle non négligeable dans la dynamique d'installation des arbres. Elle pourrait expliquer, en partie du moins, ces dates précoces, d'autant plus que ce secteur pouvait jouir, comme aujourd'hui, de conditions climatiques particulièrement favorables.

La position de la zone pollinique h (Subboréal ou Subatlantique ?) suscite également des interrogations. Les indices pastoraux en forte hausse et le déclin progressif du couvert forestier pourraient très bien s'inscrire dans 


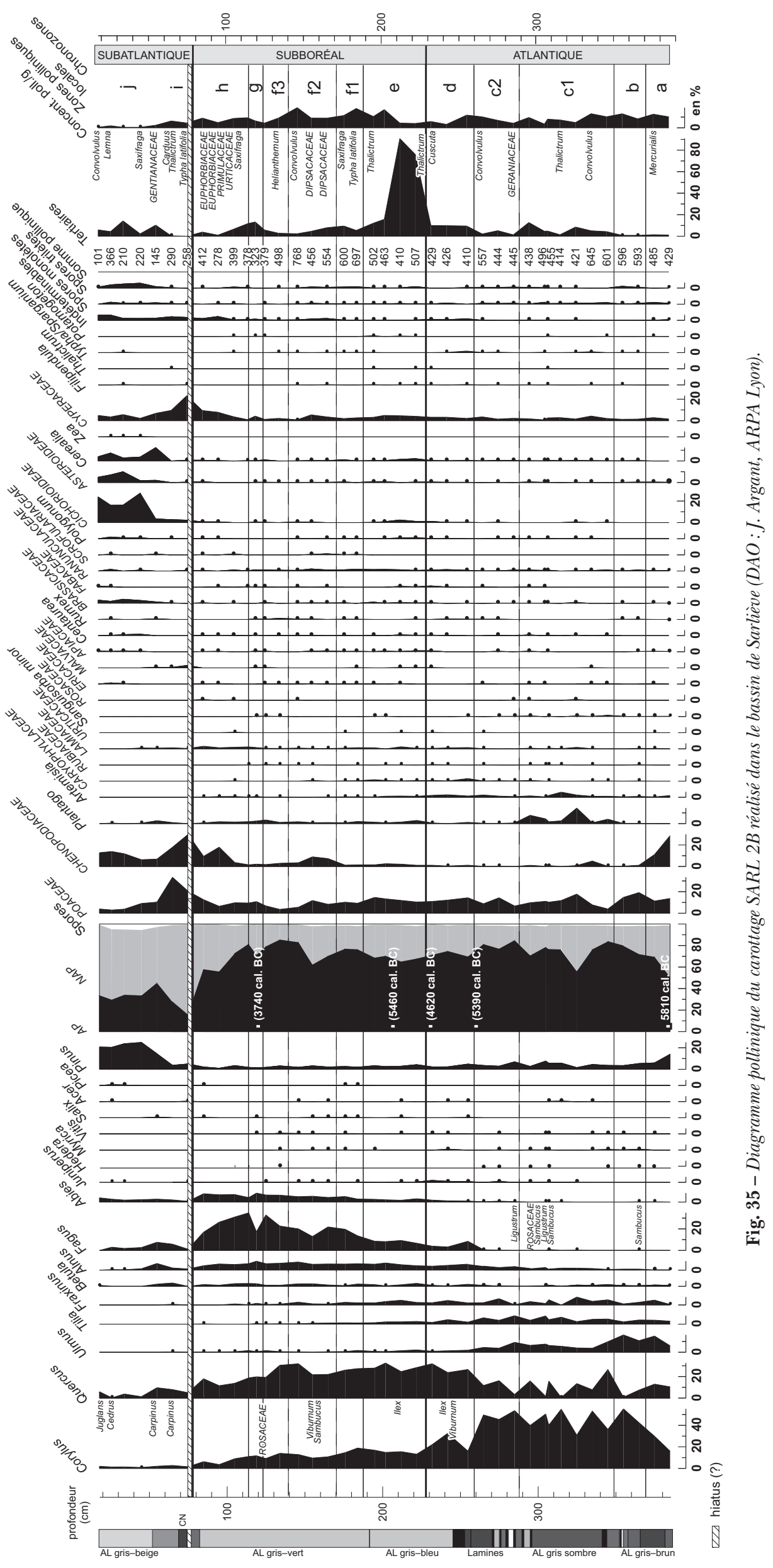




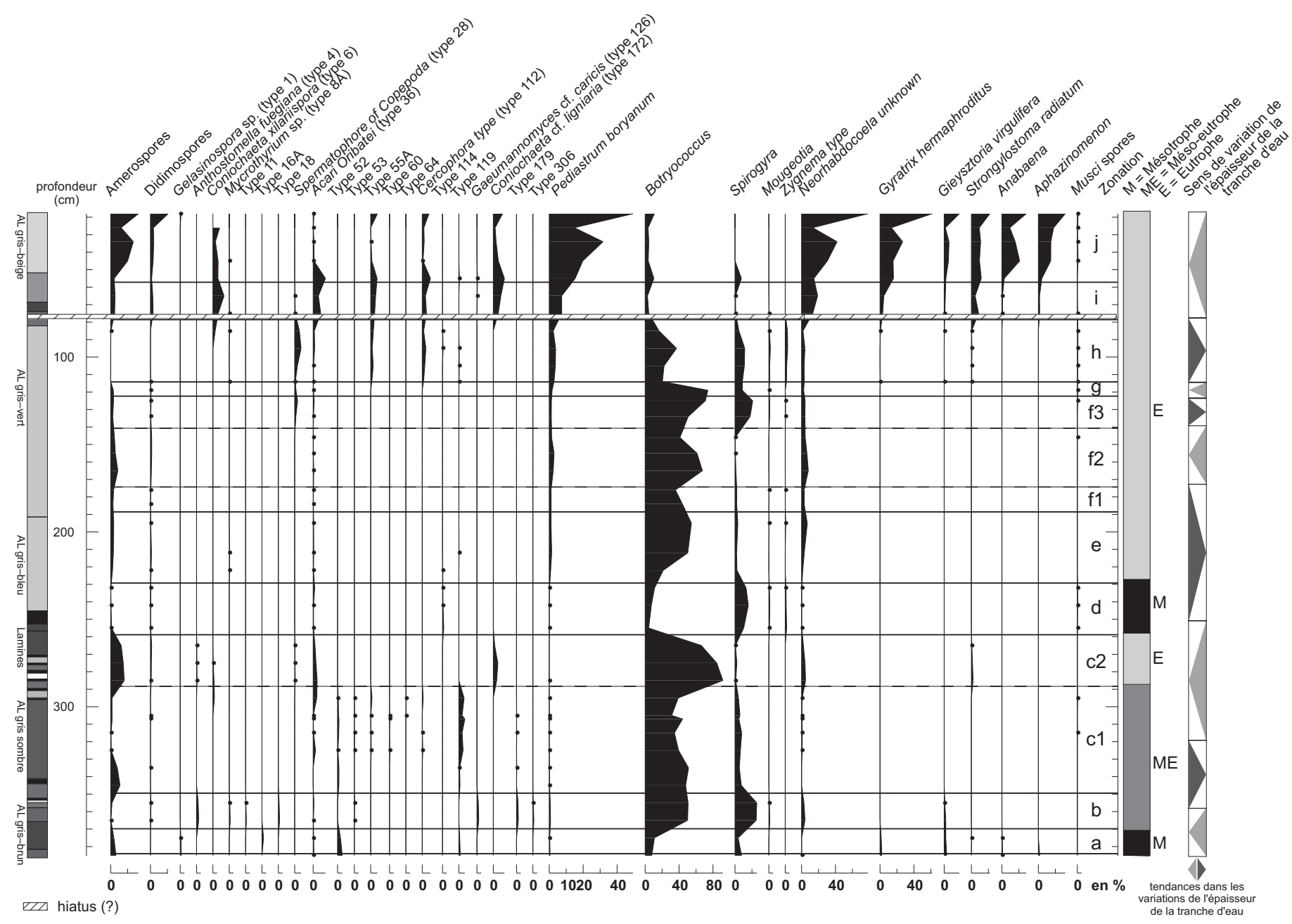

Fig. 36 - Diagramme des microfossiles non polliniques du carottage SARL $2 B$ réalisé dans le bassin de Sarliève (Analyse : J. A. López Sáez, Université de Madrid; DAO :J. Argant, ARPA Lyon).

le Subatlantique. Dans ces conditions, un hiatus existerait entre g (zone datée 3740 cal. B.C.) et h, dont le début devrait alors théoriquement se situer vers 850 cal. B.C. Mais aucune rupture profonde ni durable n'apparaît dans le diagramme. Le maintien du hêtre et du sapin d'une part, l'homogénéité du dépôt argilo-limoneux gris-vert entre $84 \mathrm{~cm}$ et $192 \mathrm{~cm}$ d'autre part, conduisent à placer cette période $\mathrm{h}$ dans la continuité du Subboréal. Cette hypothèse, compatible avec la date 3740 cal. B.C., est donc retenue pour l'instant.

En attendant de nouvelles données, le cadre chronologique admis pour l'ensemble des interprétations reste basé sur les résultats de l'analyse pollinique, ces derniers offrant une image non perturbée de l'évolution de la végétation de l'Atlantique moyen à la fin du Subboréal.

\section{Les structures archéologiques}

\section{Le problème de la conservation du pollen en contexte archéologique}

Onze structures (des puits, des fossés et une coupe stratigraphique) situées dans les secteurs 1, 2 et 3 ont été testées à travers 43 analyses. Seuls neuf spectres issus du remplissage de trois fossés différents sont considérés comme fiables (nombre de grains comptés généralement supérieur à 300) et sont retenus (fig. 38).

L'irrégularité dans la quantité de pollen préservé dans les différentes couches peut s'expliquer par diverses conditions de dépôt liées à la morphologie et à la fonction de la structure archéologique, ainsi qu'à son histoire: curage, comblement rapide ou lent, volontaire ou naturel. 


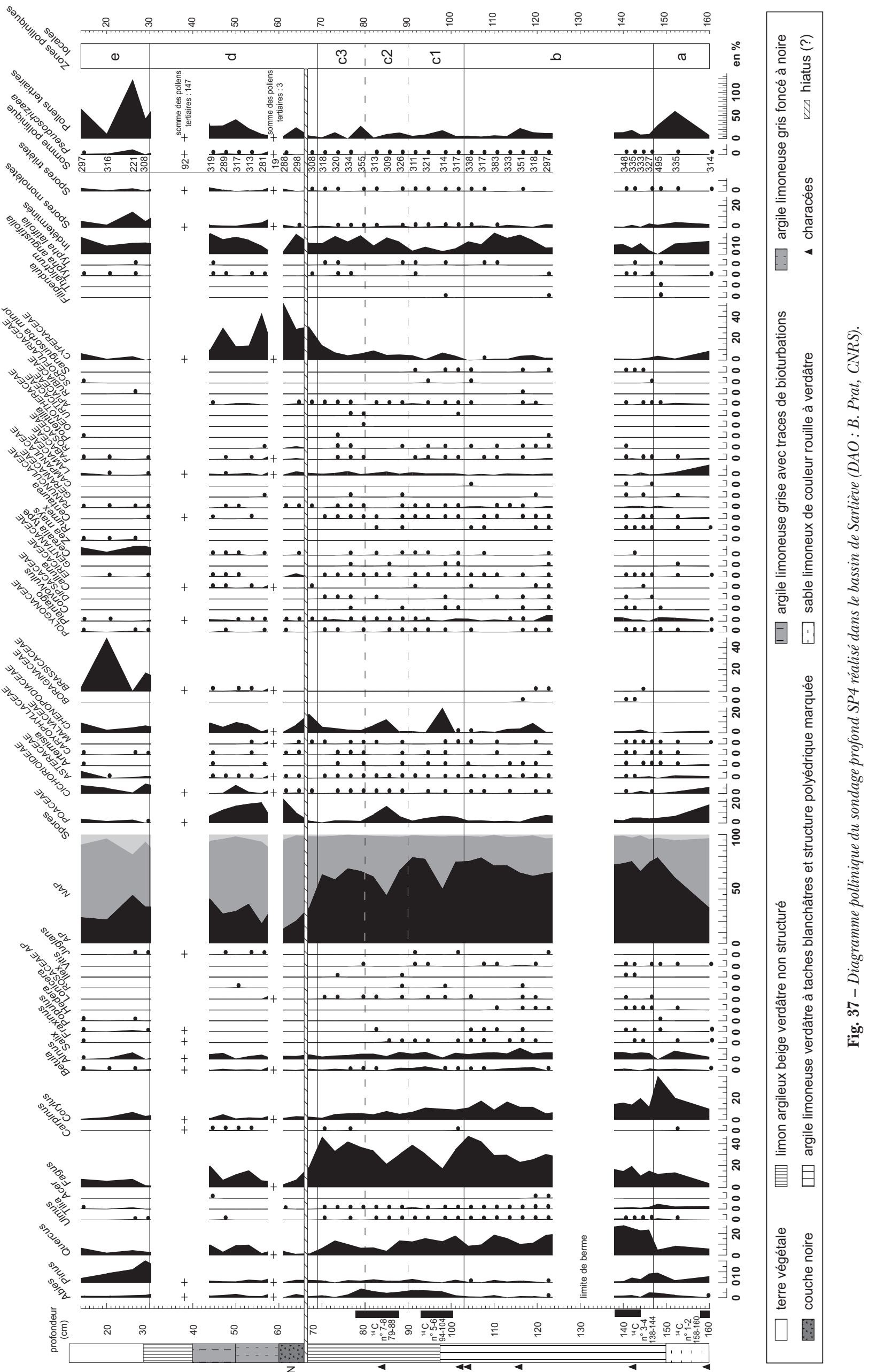


Tabl. II - Corrélations entre les zones polliniques des diagrammes SARL 2B et SP4 du bassin de Sarliève.

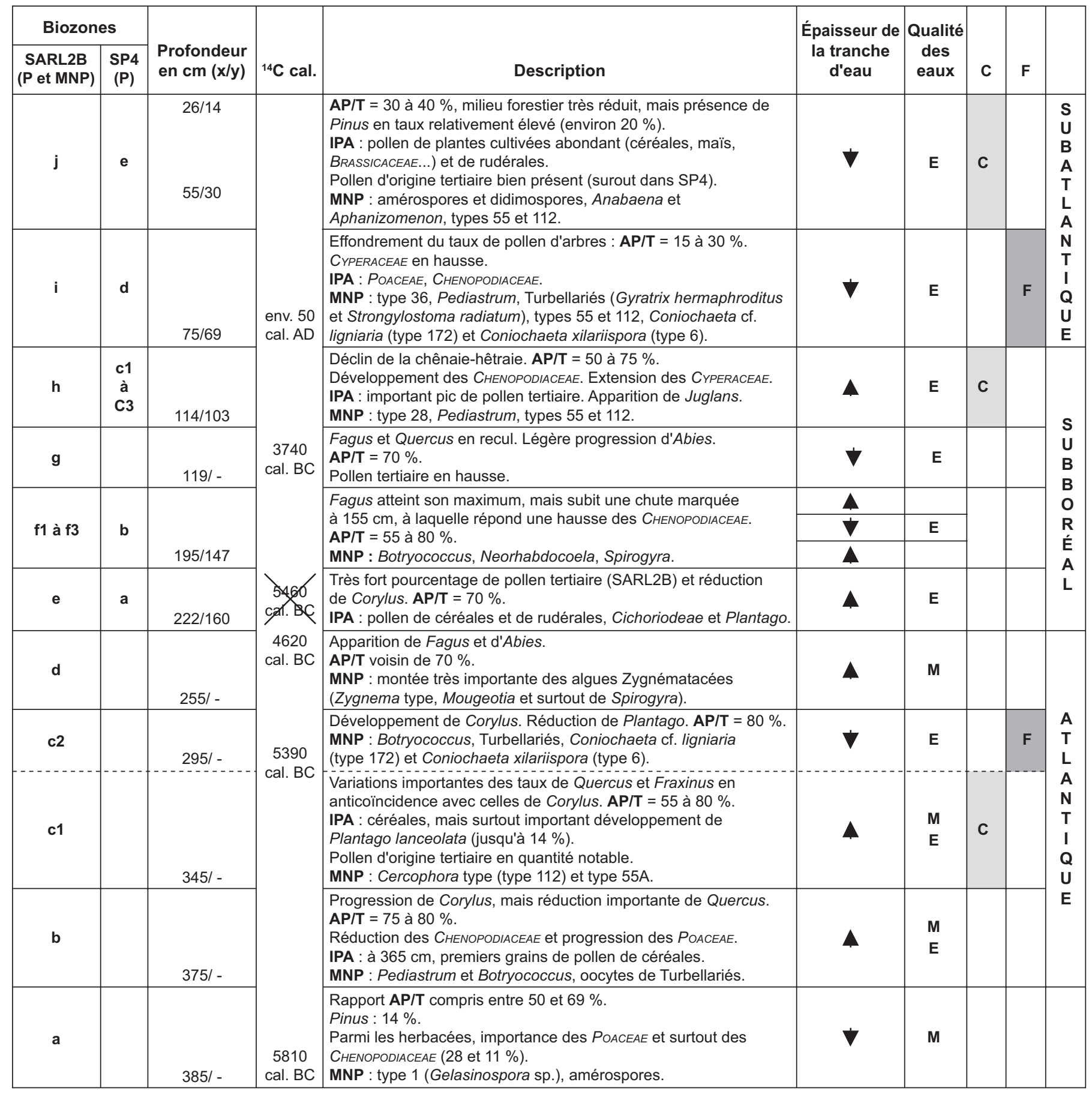

\begin{tabular}{|llllll}
\hline $\mathbf{P}$ : pollen & $\mathbf{x}:$ SARL2B & AP/T : taux de pollen d'arbres & $\mathbf{A}:$ plus élevée & $\mathbf{M}:$ mésotrophe & $\mathbf{C}:$ coprophile \\
MNP : microfossile non pollinique & $\mathbf{y}:$ SP4 & IPA : indice pollinique d'anthropisation & $\mathbf{\nabla}:$ moins élevée & $\mathbf{E}:$ eutrophe & $\mathbf{F}:$ carbonicole (feu) \\
\hline
\end{tabular}




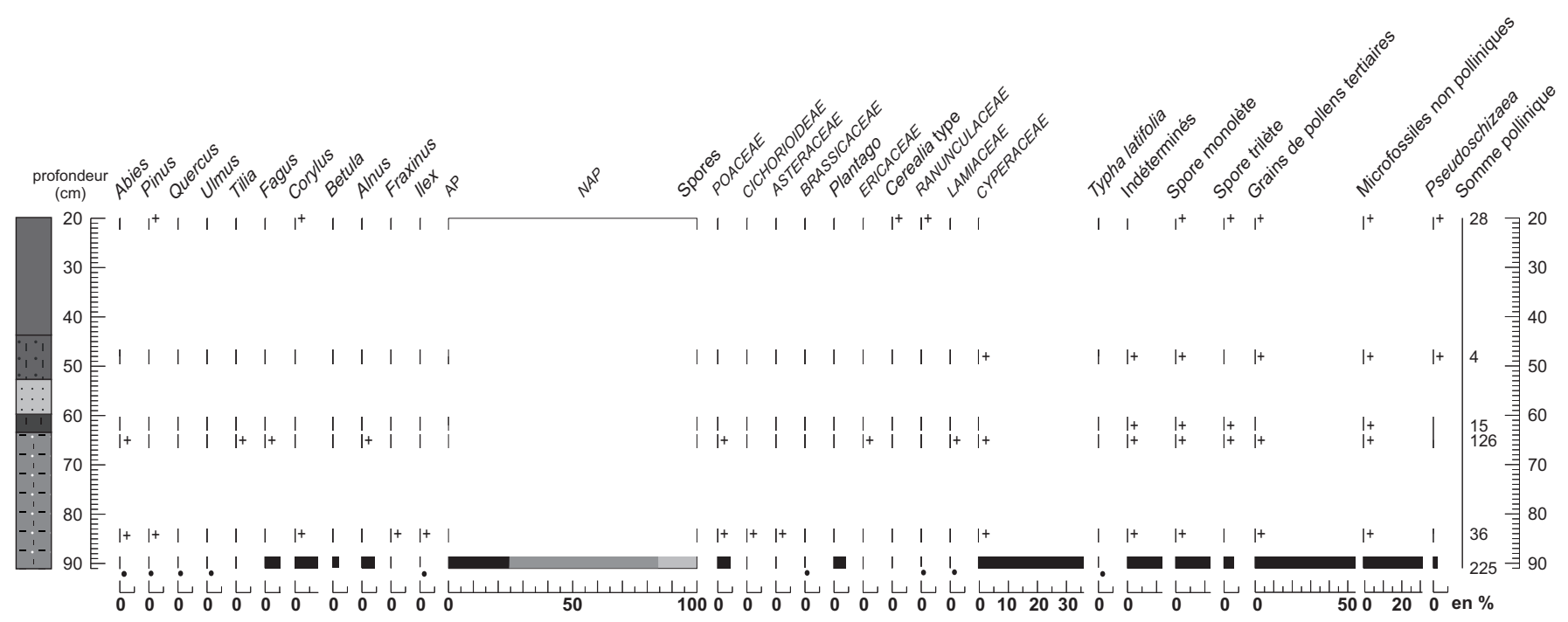

terre végétale (US1) $\quad \cdots$ sables grossiers et gravillons (US3)

limons sableux marron homogènes à petit cailloutis (US2)
-- limons sableux gris-brun, nombreuses coquilles (US13)

limons sableux noirs, niveau archéologique (US4)

Paléolac de Sarliève. Secteur 1, sondage 1109, fossé F168 (60-120 AD)

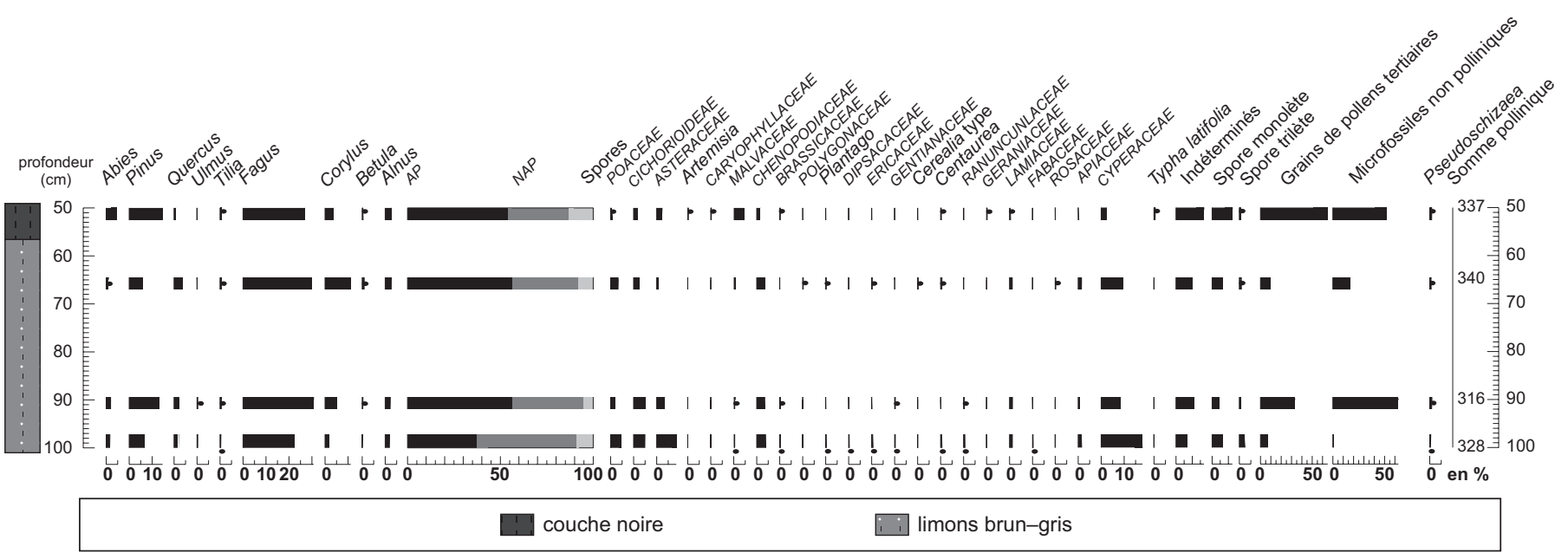

Paléolac de Sarliève. Secteur 2, sondage 190, fossé f298
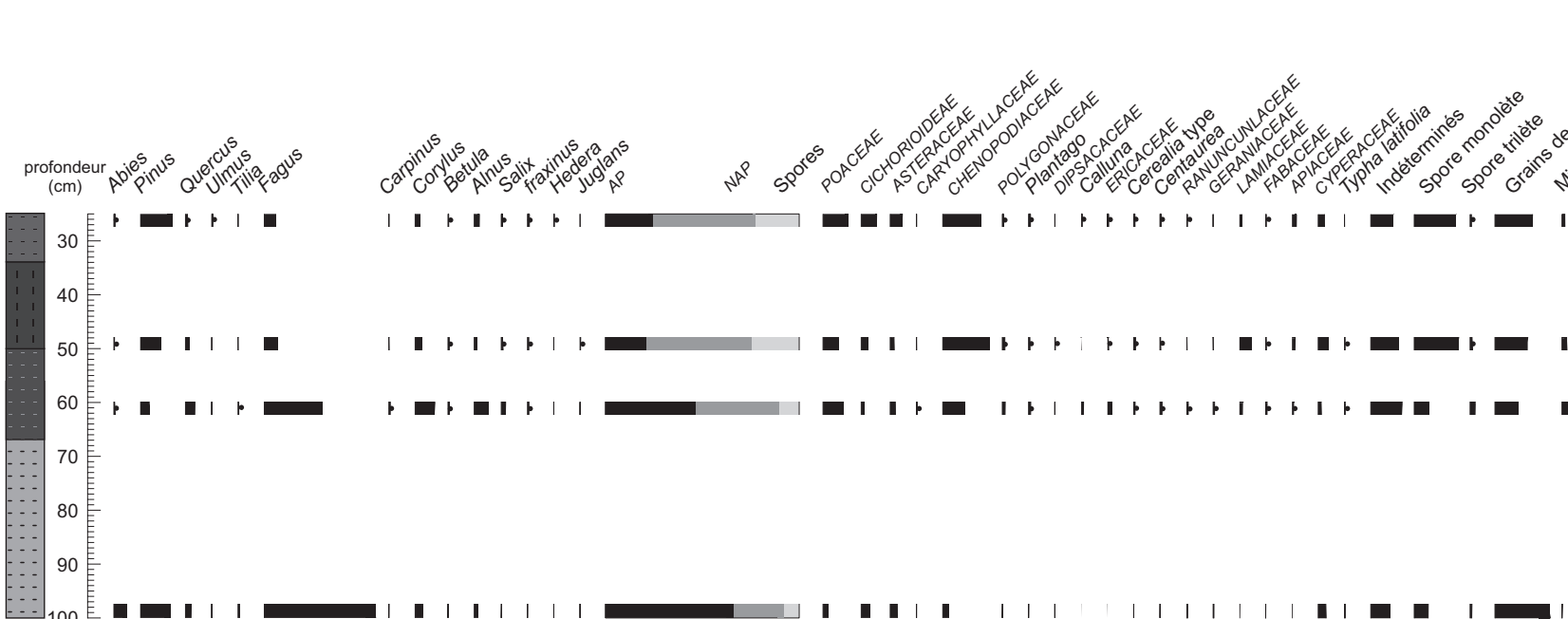

口 I |

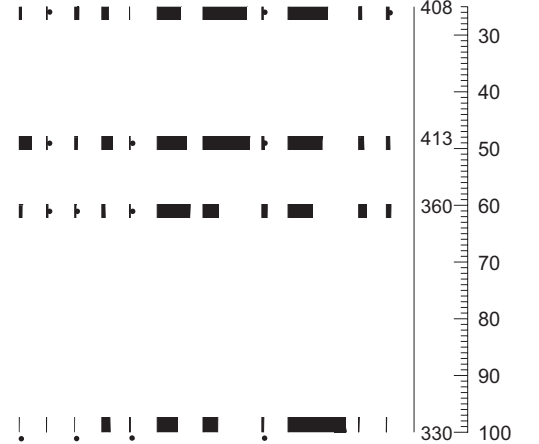

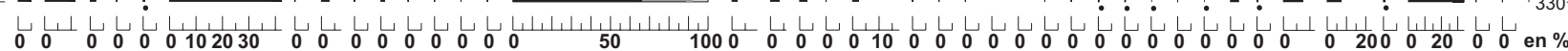

Fig. 38 - Diagrammes polliniques de trois structures archéologiques de la Grande Halle à Sarliève (DAO : B. Prat, CNRS). 


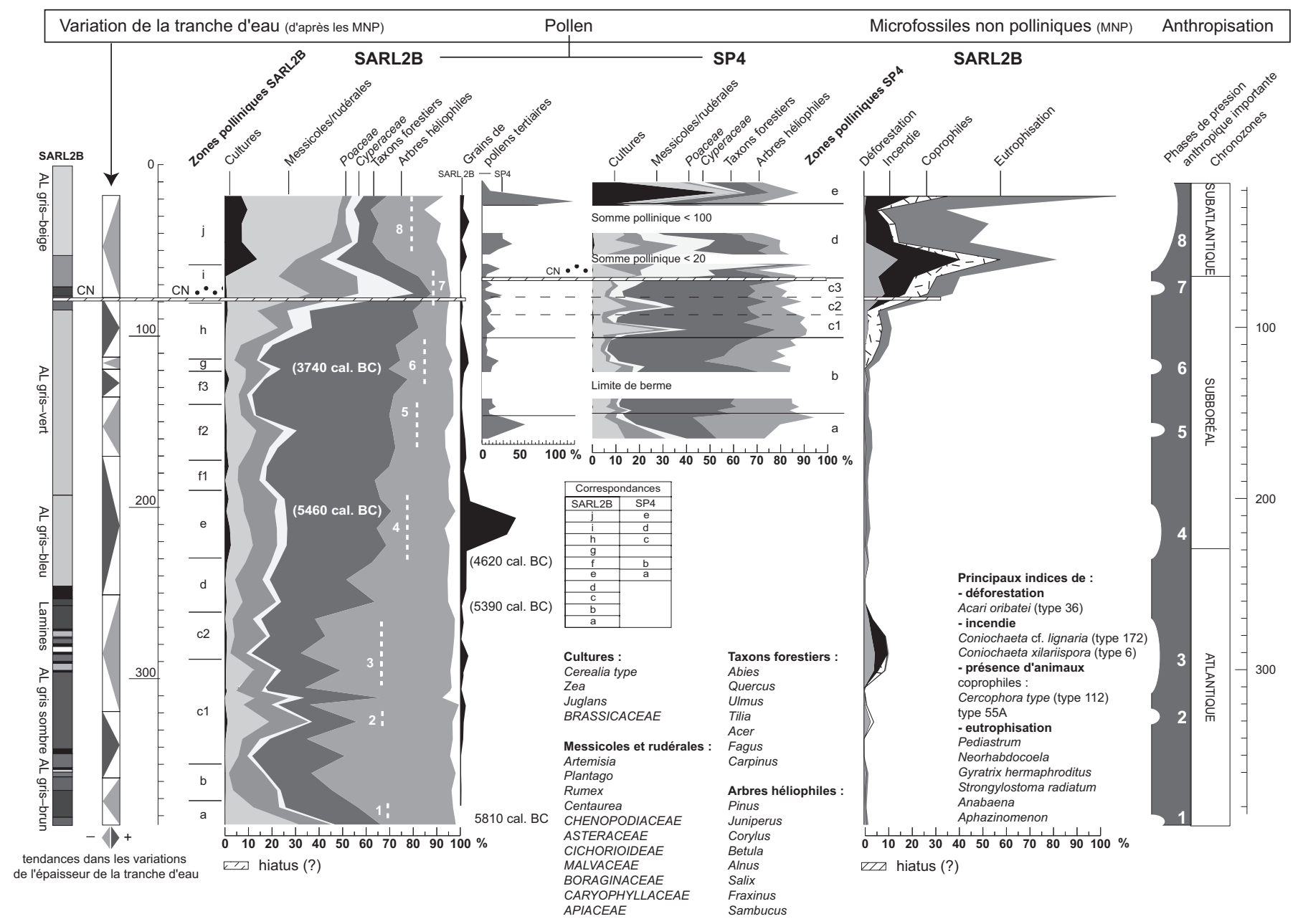

Fig. 39 - Synthèse des données paléoenvironnementales du bassin de Sarliève : palynologie (J. Argant et B. Prat) et microfossiles non polliniques (J. A. López Sáez) (DAO :J. Argant, ARPA Lyon, B. Prat, CNRS et J. A. López Sáez, Université de Madrid).

Elle peut avoir aussi pour cause des conditions de conservation variables du pollen, en fonction de la position des structures par rapport au lac et à la nappe phréatique (Havinga, 1971 et 1984 ; Marguerie, 1992 ; Prat, 2006).

\section{Les données}

Secteur 1, sondage 1109, fossé F168

Cette structure est située au bord de la limite ouest du secteur 1 (fig. 38). Un seul spectre, obtenu à la base du remplissage du fossé (à $91 \mathrm{~cm}$ ), est exploitable, du fait de la proximité de la nappe phréatique. Il indique un milieu humide ouvert où perdurent des îlots forestiers avec Fagus accompagné de Quercus, Tilia, Ulmus, Abies, Ilex et Corylus, qui est le taxon dominant.
Une bonne concordance peut être établie avec la zone pollinique SP4.d, comme en témoignent les taux de pollen d'arbres similaires et la prédominance des Cyperaceae, ainsi que l'âge voisin de ces niveaux: la couche noire correspondant à SP4 est datée entre 49 cal. B.C. et 81 cal. A.D. par le radiocarbone dans le carottage SARL 2. Grâce à la céramique de Lezoux type Déchelette 74 (information orale: A. Wittman), la base du remplissage du fossé peut être datée entre 60 et 120 apr. J.-C.

\section{Secteur 2, sondage 190, fossé F298}

Le fossé F298 situé dans le secteur 2, au cœur du delta, à proximité de $\mathrm{SP} 4$, enregistre aussi à sa base un paysage ouvert où les rudérales (Chenopodiaceae, Cichorioideae et 
surtout Asteraceae) apparaissent nettement (fig. 38). Une occurrence de Cerealia est à noter à $62 \mathrm{~cm}$ ainsi qu'un taux relativement fort de Malvaceae $(5 \%)$ à $52 \mathrm{~cm}$ (couche noire). La séquence pollinique de ce fossé pourrait être rattachée à la zone SP4.c précédant la chute du pollen d'arbre et le développement de la cypéraie (fig. 37).

\section{Secteur 3, sondage 189, fossé F402}

Ce fossé, localisé dans le secteur 3, est situé sur la bordure ouest de l'emprise de la Grande Halle. La hêtraie forme l'essentiel de la partie boisée, bien représentée à la base (fig. 38). Dans les trois échantillons les plus récents, les arbres reculent nettement, évolution due aux coupes effectuées aux dépens de Fagus. Alnus et Corylus, ainsi que d'autres héliophiles tels que Betula, Salix, Fraxinus et surtout Pinus, profitent momentanément de la réduction de la hêtraie. La représentation des Poaceae et des rudérales (Plantago, Cichorioideae, Asteraceae, Polygonum et surtout Chenopodiaceae) augmente. Cerealia type est à signaler. La séquence du fossé F402 pourrait correspondre à la zone SP4.d.

\section{RYTHMES ET MODALITÉS DE L'ANTHROPISATION DE LA VÉGÉTATION}

L'analyse pollinique met en évidence les effets sur la végétation d'une occupation permanente des abords du lac depuis 8000 ans (fig. 39). Si elle est continue, la pression anthropique n'est pas nécessairement toujours très forte. Pendant toute la durée de l'Atlantique et du Subboréal, il semble que les activités humaines aient été limitées à l'ouverture de clairières exploitées temporairement. C'est à partir de la fin du Subboréal que commence véritablement la réduction du manteau forestier. Celle-ci devient maximale à l'époque romaine. La corrélation des diagrammes avec la courbe du pollen d'origine tertiaire montre un lien entre la déforestation et le transport puis le dépôt de ce pollen ancien, ce qui implique une action érosive accrue sur les versants.

Huit temps forts peuvent être soulignés :

- pendant l'Atlantique, on assiste à l'attaque de la chênaie, surtout au profit des pâturages (phases 1, 2 et 3) et des cultures de céréales (phase 4) qui peuvent être attribués au Néolithique ;

- pendant le Subboréal, la corylaie se réduit et les pâturages s'étendent (phases 5 et surtout 6) : il reste impossible de déterminer si ce phénomène se produit à l'âge du Bronze ou à l'âge du Fer ;
- au Subatlantique apparaît une accentuation du déboisement, puis un essor important des cultures (phase 7 à l'époque romaine et/ou médiévale, phase 8 à l'époque moderne).

$$
\text { J. A., J. B. et J. A. S. L. }
$$

\section{CARPOLOGIE}

L'étude carpologique permet d'affiner la connaissance des cultures et des pratiques agricoles du second âge du Fer à la période augustéenne, et d'apporter des informations complémentaires sur l'état du marais. Les structures échantillonnées sont les suivantes (tabl. III et IV) :

- F2101, couche 3 datée de la transition La Tène B2/C1 (300-250 av. J.-C.) ;

- F2102, couche 3 datée de la transition La Tène B2/C1 et La Tène C1 (vers 250 av. J.-C.) ;

- F2102, couche 1 datée de La Tène C1 (250-200 av. J.-C.) ;

- Puits 3057 daté de la transition La Tène C2/D1 (160-100 av. J.-C.) ;

- Puits 2460 et 2485 datés de La Tène D2 (60-40 av. J.-C.).

\section{L'ÉVOLUTION DU MILIEU DU SECOND ÂGE DU FER AU DÉBUT DE L'ÉPOQUE ROMAINE}

\section{Formation « de marais et de bord des eaux »}

À la transition La Tène B2/C1 (300-250 av. J.-C.), l'amplitude écologique étroite, inféodée au bord des étangs, du scirpe lacustre (Scirpus lacustris), plante hélophyte, montre une formation de marais et de rives. Vers 250 av. J.-C., l'œnanthe aquatique (Oenanthe aquatica) s'ajoute au scirpe lacustre. À La Tène C1 (250-200 av. J.-C.), trois taxons sont présents, par ordre d'abondance: le scirpe lacustre, le pâturin commun (Poa trivialis), qui pousse sur des prairies humides, et le lycopode d'Europe (Lycopus europaeus), qui se développe plutôt dans les marais et les aulnaies. À la période de transition La Tène C2/D1 (160-100 av. J.-C.), seul le scirpe lacustre est attesté.

\section{Formation « de bois, de haies et de fourrés "}

Les carporestes d'écologie du type «bois, haies, fourrés » sont peu représentés (quelques fragments de noisette). L'étude anthracologique en cours, réalisée par M. Cabanis, complètera l'histoire de la forêt à cette période. Elle confirme déjà l'existence d'un espace ouvert et fortement déboisé (essences héliophiles et cernes larges), avec certainement une gestion sylvicole. 
Tabl. III - Datation des prélèvements carpologiques dans le bassin de Sarliève.

\begin{tabular}{|l|l|l|}
\hline \multicolumn{2}{|c|}{ Datations } & \\
\hline \multirow{3}{*}{ Gallo-romain } & Gallo-romain sans précision & bât 2196 pièce B, US5 \\
\cline { 2 - 3 } & début période augustéenne & puits F 2249 \\
\hline \multirow{3}{*}{ Second Âge du Fer } & La Tène D2 (60-40 av. J.-C.) & $\begin{array}{l}\text { puits F 2485 ; puits 2460 ; dans l'ensemble La Tène D1 } \\
\text { tesson La Tène D2 }\end{array}$ \\
\cline { 2 - 3 } & $\begin{array}{l}\text { transition La Tène C1 et La Tène D2 (200-60 av. J.-C.), } \\
\text { plus proche de La Tène D2 que de La Tène C1 }\end{array}$ & F 2103 couche 3; F 2103 couche 4 \\
\cline { 2 - 3 } & transition La Tène C2/D1 (160-100 av. J.-C.) & puits 3057 \\
\cline { 2 - 3 } & La Tène C1 (250-200 av. J.-C.) & F 2102 couche 1 \\
\cline { 2 - 3 } & transition entre La Tène B2/C1 et La Tène C1 (250 av. J.-C.) & F 2102 couche 3 \\
\cline { 2 - 3 } & transition La Tène B2/C1 (300-250 av. J.-C.) & F 2101 couche 3 \\
\hline
\end{tabular}

Tabl. IV - Nombre de carporestes par période chronoculturelle dans le bassin de Sarliève : LT, La Tène.

\begin{tabular}{|c|c|c|c|c|c|c|c|}
\hline & & & & & & & \\
\hline & & \begin{tabular}{|l|} 
Transition \\
LT B2/C1
\end{tabular} & $\begin{array}{c}\text { Entre la transition } \\
\text { LT B2/C1 } \\
\text { et LT C1 }\end{array}$ & LT C1 & \begin{tabular}{|l|} 
Transition \\
LT C2/D
\end{tabular} & \begin{tabular}{|c|} 
Entre LT C1 \\
et LT D2
\end{tabular} & LT D2 \\
\hline & Nombre de prélèvements & 1 & 1 & 1 & 1 & 2 & 2 \\
\hline Céréales & Type de reste & & & & & & \\
\hline Orge polystique vêtue (Hordeum vulgare) & semence & 20 & & 10 & 44 & 20 & 11 \\
\hline Orge polystique vêtue (Hordeum vulgare) & rachis & & 1 & & & & \\
\hline Blé tendre/dur (Triticum aestivum/durum) & semence & 10 & & 8 & 2 & 4 & 32 \\
\hline Blé tendre/dur (Triticum aestivum/durum) & semence fragmentée & & & & & 4 & \\
\hline Blé tendre/dur (Triticum aestivum/durum) & rachis & 1 & 3 & 2 & & & \\
\hline Amidonnier (Triticum dicoccum) & semence & 24 & 2 & 6 & 34 & 21 & \\
\hline Amidonnier (Triticum dicoccum) & semence fragmentée & 106 & & 19 & & & \\
\hline Amidonnier (Triticum dicoccum) & furca, base d'épillet & 110 & 30 & & 42 & 46 & 50 \\
\hline Engrain (Triticum monococcum) & semence & 11 & 6 & & 9 & 3 & 1 \\
\hline Engrain (Triticum monococcum) & semence fragmentée & 77 & & & & & \\
\hline Engrain (Triticum monococcum) & furca, base d'épillet & 1 & 230 & 108 & 15 & 82 & 13 \\
\hline Épeautre (Triticum spelta) & semence fragmentée & & & & & 15 & \\
\hline Épeautre (Triticum spelta) & furca, base d'épillet & & 15 & 12 & 6 & & \\
\hline Millet commun (Panicum miliaceum) & & & & 5 & & 21 & \\
\hline \multicolumn{8}{|l|}{ Légumineuses } \\
\hline Ers (Vicia ervillia) & semence & & & & & & 1 \\
\hline Féverole (Vicia faba) & semence & & 2 & & & 2 & \\
\hline Lentille (Lens culinaris) & semence & 14 & & 1 & 9 & 6 & \\
\hline Pois (Pisum sp.) & semence & & & & & 2 & \\
\hline Vesce cf. cultivée (Vicia cf. sativa) & semence & 4 & 6 & 18 & 11 & 6 & 2 \\
\hline Nombre d'espèces cultivées & & 4 & 5 & 6 & 5 & 6 & 4 \\
\hline \multicolumn{8}{|l|}{ Plantes cueillies } \\
\hline Noisetier (Corylus avellana) & fragment coque ou péricarpe & 9 & 29 & 2 & 1 & 1 & \\
\hline
\end{tabular}




\section{LES PRATIQUES AGRICOLES GAULOISES ET GALLO-ROMAINES}

\section{Les données}

Les espaces cultivés peuvent être reconstitués grâce aux plantes domestiques, aux parasites des céréales, aux produits dérivés d'origine végétale (bouillies, galettes) et aux adventices de cultures.

\section{Plantes et produits dérivés domestiques}

L'assemblage carpologique de Sarliève s'inscrit dans le cadre régional des plantes cultivées trouvées au second âge du Fer et à l'époque romaine en Auvergne (Cabanis, 2002). Le blé amidonnier (Triticum dicoccum) est présent à toutes les périodes de La Tène, de 300 à 60 av. J.-C., et durant la période augustéenne. L'orge polystique vêtue (Hordeum vulgare), deuxième céréale à être cultivée en importance, est attestée dans toutes les structures étudiées. Le millet commun (Panicum miliaceum) est la troisième céréale la plus répandue. Sa culture est mentionnée aux âges du Fer en Grande Limagne (Bouby, 1993). Le millet commun, attesté au premier âge du Fer, connaît un fort développement au second âge du Fer en France méridionale (Marinval, 1988). Enfin, l'engrain (Triticum monococcum), le blé nu (Triticum aestivum/durum) et l'épeautre (Triticum spelta) sont reconnus durant ces périodes.

Deux sclérotes d'ergot (Claviceps sp.) ont été déterminées dans les couches 3 et 4 de la structure F2103 (20060 av. J.-C.) et dans le puits F2485 (60-40 av. J.-C.). Une farine contenant $5 \%$ à $7 \%$ d'ergots peut entraîner la mort de la personne qui la consomme (Jean-Blain, 1973).

La lentille (Lens culinaris), la fève (Vicia faba) et le pois (Pisum sativum) prennent de l'importance à l'époque romaine en Auvergne, à Sarliève en particulier. Une réserve de fèves a même été découverte dans un des bâtiments gallo-romains (bâtiment B de [CO-085], US5, fig. 20). La vesce cultivée (Vicia sativa) a été trouvée dans tous les échantillons analysés.

La période de transition La Tène B2/C1 (300-250 av. J.-C.) a livré douze fragments de galettes. Un fragment de bouillie de millet a également été identifié dans le puits F2485 daté de La Tène D2.

\section{Adventices de cultures}

Cinq types d'associations végétales se retrouvent à Sarliève : les adventices de cultures, les friches et décombres, les prairies, les berges et eaux, et enfin les formations

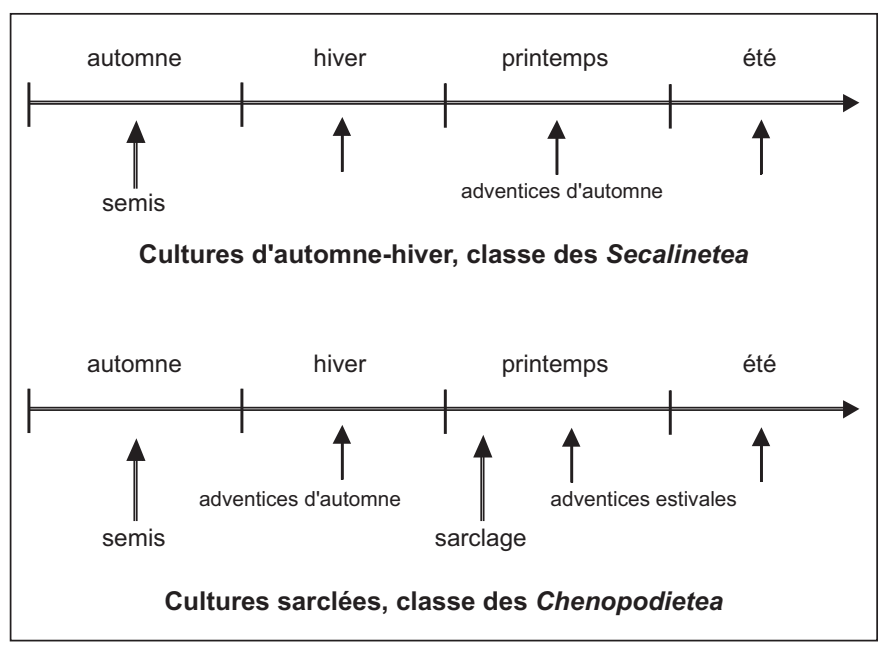

Fig. 40 - Calendrier agricole du bassin de Sarliève (DAO : M. Cabanis, CNRS).

boisées. Les adventices de cultures se composent de deux types d'association: les cultures de céréales d'automne/ hiver, de moissons (classe des Secalinetea), et les cultures de printemps/été, sarclées (classe des Chenopodietea) (fig. 40).

\section{Discussion}

Grâce aux résultats des analyses factorielles des correspondances (AFC), les milieux de vie sont corrélés aux différentes périodes. On peut ainsi reconstituer les modalités culturales et comprendre à quelle saison ont eu lieu les semailles (fig. $41, \mathrm{n}^{\text {os }} 1$ et 2 ).

\section{De La Tène B2 à La Tène C1 (300-200 av.J.-C.)}

Troiséchantillons sur quatre provenant de F2101/02/03, datés entre 300 et 200 av. J.-C., évoquent des plantes messicoles (Secalinetea) et des plantes associées aux friches, murs et décombres (fig. $42, \mathrm{n}^{\circ} 1$ ). Le quatrième échantillon, vers 250 av. J.-C., suggère plutôt des cultures sarclées au printemps. Au sein des cultures présentes à cette période, il existe une gamme d'adaptation assez large des variétés d'hiver et de printemps (Marinval, 1988). Certaines espèces comme le millet commun, la lentille et la vesce cultivée sont strictement de printemps en raison de leur sensibilité au gel. Le blé nu est plutôt semé à l'automne et passe la mauvaise saison à l'état de plantule. La présence de plantes cultivées de printemps et d'automne et des deux groupements d'adventices (Chenopodietea et Secalinetea) reflète des cultures d'hiver et d'été de La Tène B2 à La Tène C1. 
Points-lignes et points-colonnes (axes F1 et F2 : $100 \%$ )

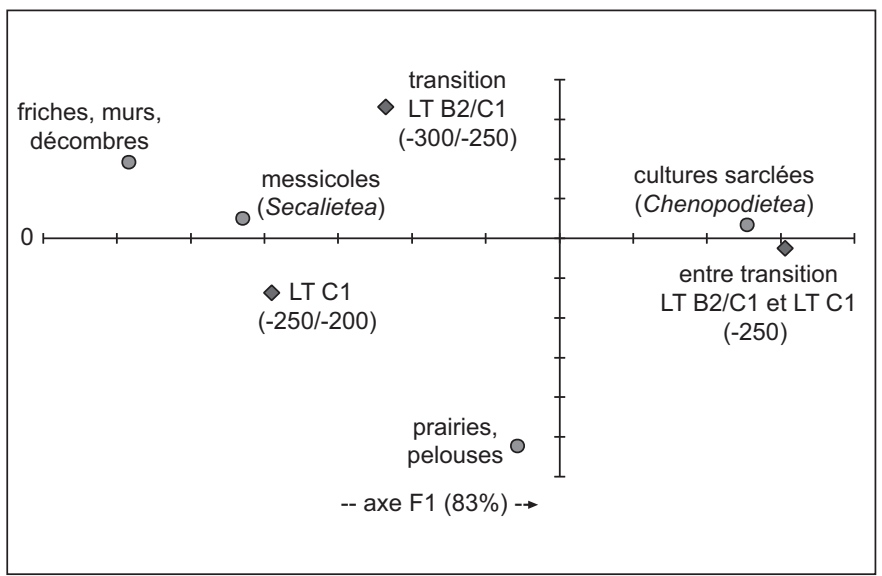

1
Points-lignes et points-colonnes (axes F1 et F2 : $100 \%$ )

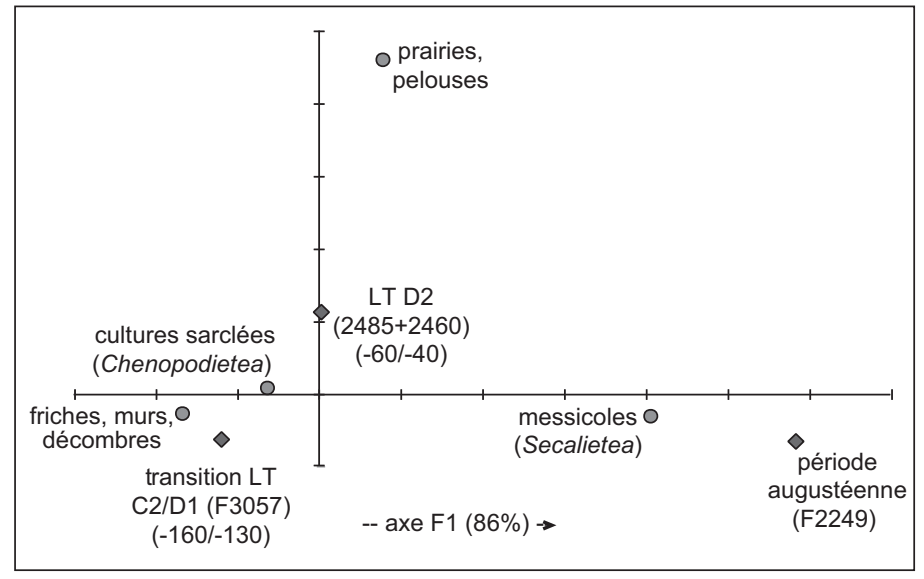

2

Fig. 41 - Carpologie du bassin de Sarliève. Analyse factorielle des correspondances (AFC) selon le nombre de restes corrigés par périodes (LT, La Tène) : 1, structures F2101 c3, F2102 c3 et F2102 c1 ; 2, puits 3057, 2460, 2485 et 2249 (DAO : M. Cabanis, CNRS).

\section{De La Tène C2 à la période augustéenne (160 av.J.-C. - début I $I^{\text {rr }}$ s. apr.J.-C.)}

Dans les puits du second âge du Fer (3057, 2460, 2485), datés de 160 à 40 av. J.-C., les cortèges de mauvaises herbes montrent une tendance aux cultures sarclées au printemps (fig. 42, $\mathrm{n}^{\mathrm{O}}$ 2). La présence de la vesce, de la lentille et de l'ers (Vicia ervilia) va dans le sens d'un semis printanier. L'orge, l'amidonnier, l'engrain et le blé nu dévoilent une gestion mixte des cultures, de printemps ou d'automne.

Dans le puits daté de la période augustéenne (2249), les adventices d'automne (Secalinetea) sont liées à des histogrammes où aucune plante ne domine significativement. La diversification du corpus des plantes cultivées par rapport au second âge du Fer est symptomatique de la romanisation.

M. C. et P. M.

\section{SYNTHÈSE SUR LES INTERACTIONS SOCIÉTÉS-MILIEUX DANS LE BASSIN DE SARLIÈVE}

Il convient à ce stade de croiser les résultats des différentes disciplines ou spécialités, afin d'esquisser un schéma évolutif global du géosystème, puis de l'« anthroposystème " constitué par le bassin de Sarliève (pl. III, hors texte).

\section{FORMATION ET FONGTIONNEMENT DU GÉOSYSTÈME LACUSTRE}

\section{LA FORMATION DU PALÉOLAC DE SARLIÈVE}

L'affaissement du substrat basal vers l'ouest et sa cote élevée au niveau de l'exutoire pourraient accréditer l'hypothèse d'une origine néotectonique de la dépression, liée au rejeu des grandes failles nord-sud de la Limagne favorisé par le volcanisme actif du Tardiglaciaire et du début de l'Holocène. La chronologie et les causes de ce phénomène restent à éclaircir.

La première mise en eau de la cuvette est un peu antérieure à la retombée volcanique CF1, datée vers 11750 cal. B.C. (Miallier et al., 2004). Elle remonterait au Bölling. L'effet de barrage dû à la remontée du substrat au nord de la dépression a été renforcé par les coulées boueuses successives issues des versant orientaux mises en évidence à l'exutoire (fig. 6).

\section{L'EMPREINTE DU VOLCANISME}

L'empreinte du volcanisme est marquée dans les sédiments par le dépôt d'une retombée de cendres bien connue des géologues, car présente dans toute la Grande Limagne: le complexe téphrique CF1, dont l'émission, datée en moyenne de 11750 cal. B.C. et issue d'un magma trachyandésitique basaltique, est attribuée au puy de la 


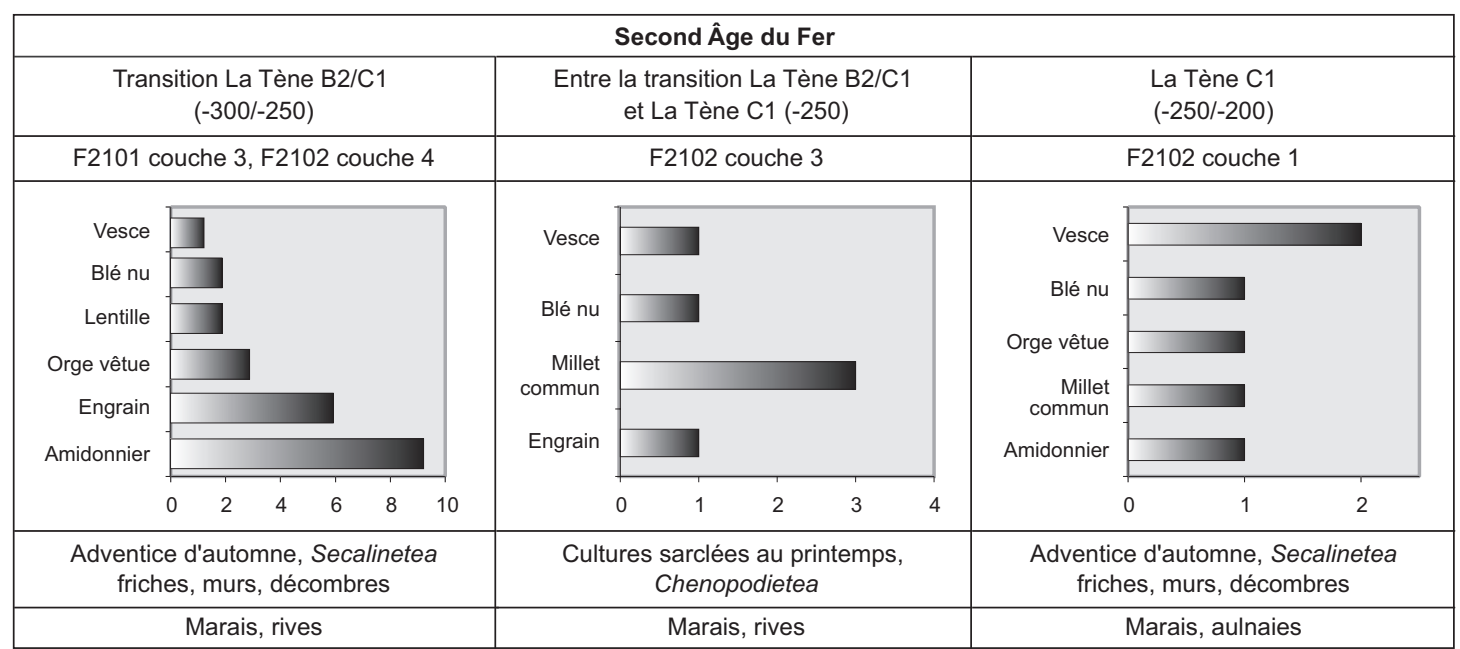

1

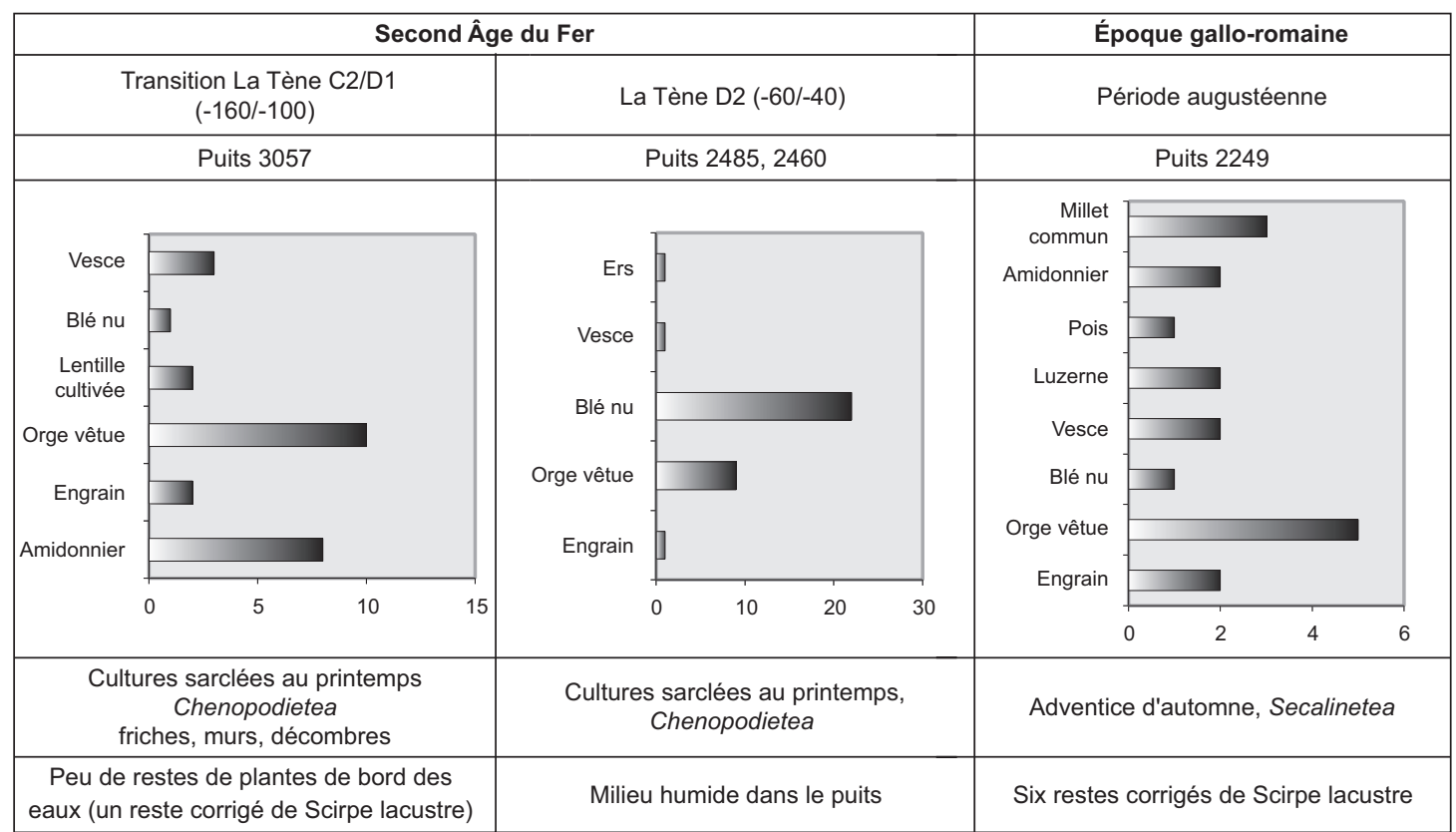

2

Fig. 42 - Synthèse des résultats carpologiques du bassin de Sarliève :

1, structure F2101/02/03 ; 2, puits 3057, 2460, 2485 et 2249 (DAO : M. Cabanis, CNRS).

Nugère (Vernet, Raynal, 2000). Un nouveau marqueur du volcanisme trachytique de la chaîne des Puys, postérieur au précédent, a été mis en évidence dans le remplissage du paléolac: le "téphra de Sarliève ", dont l'âge précis et le volcan émetteur sont en cours d'étude, mais dont l'originalité géochimique est confirmée par les analyses (Vernet, Raynal, 2002 ; Miallier et al., 2004 ; Fourmont et al., 2006).

\section{COMPLEXITÉ DU FONCTIONNEMENT DU GÉOSYSTÈME LACUSTRE}

Les nombreux forages et les sondages profonds ont mis en évidence quatre zones distinctes dans l'ancien lac de Sarliève, au remplissage sédimentaire différent durant le Tardiglaciaire et l'Holocène (fig. 6) : 
- deux bassins, l'un plus petit au nord, l'autre plus grand au sud, au fond plat et régulier ;

- une zone de deltas séparant ces deux bassins ;

- la zone d'exutoire ;

- les bordures du marais.

L'étude de ces diverses unités s'est avérée indispensable pour comprendre le fonctionnement global du géosystème lacustre, car elles ont stocké, chacune, une partie différente des sédiments produits par l'érosion sur les versants. En outre, il apparaît qu'elles ont joué un rôle primordial dans les choix d'implantation de l'habitat. Deux grandes phases peuvent être distinguées :

- une première phase, du Bölling à l'Atlantique, au cours de laquelle les zones de deltas et les deux bassins ont fonctionné de manière bien distincte ;

- une seconde phase, de l'Atlantique à l'actuel, pendant laquelle ces unités ont fonctionné de manière synchrone, suite à l'élévation générale du plan d'eau.

\section{FLUCTUATIONS DU PLAN D'EAU ET OCCUPATION DES RIVES}

La combinaison des données géologiques, paléoécologiques et archéologiques permet de restituer les grandes phases de l'évolution du plan d'eau et de l'occupation des rives par les communautés successives. Au sein de ce géosystème lacustre semi-endoréique, les fluctuations verticales du plan d'eau sont conditionnées, en dehors de tout aménagement anthropique, par le régime des précipitations (en particulier le bilan précipitations/évaporation), mais également par le niveau de base de l'exutoire. Or ce dernier, situé dans une zone de coulées boueuses, a pu varier sous l'influence de multiples facteurs : instabilité intrinsèque des versants marno-calcaires, pluviométrie, action de l'homme. On soulignera la coïncidence chronologique de fluctuations particulièrement importantes de la nappe d'eau avec des phases climatiques susceptibles d'expliquer, au moins en partie, ces variations (pl. III, hors texte).

\section{LE TARDIGLACIAIRE : DU BÖLLING AU DRYAS RÉCENT}

Entre la mise en eau de la cuvette vers 11750 cal. B.C. et 9550 cal. B.C. environ, la constitution de la première génération de deltas (US1) implique une phase de haut niveau relatif du plan d'eau. La tranche d'eau dépassait alors la cote 342 m NGF, tandis que le fond de la cuvette se trouvait aux environs de 338 m NGF minimum. Pendant cette période, la sédimentation a été faible dans les bassins nord et sud.

\section{L'HOLOCÈNE INFÉRIEUR : PRÉBORÉAL ET BORÉAL}

Au début de l'Holocène, la couche colluviale UC1 montre l'émersion de la zone des deltas (le toit des colluvions pédogénisées est à $343 \mathrm{~m}$ NGF environ). La pédogenèse qui l'affecte a pu se dérouler pendant l'essentiel de l'Holocène inférieur. L'enrichissement en dolomite à la partie supérieure de l'unité lacustre ULla montre le caractère endoréique des bassins et l'alternance de phases d'assèchement et de remise en eau qui les affecte. Le niveau d'eau saumâtre, peu profond et d'extension limitée, ne paraît pas avoir été très supérieur à la cote 339 m NGF.

\section{L'ATLANTIQUE : FIN DU MÉSOLITHIQUE-NÉOLITHIQUE}

Au cours de l'Atlantique (UL2), la tranche d'eau a été affectée par des fluctuations d'une amplitude plus forte qu'à la phase précédente, ainsi que par des variations de la chimie des eaux. Les variations des cortèges polliniques et des microfossiles non polliniques expriment ces fluctuations cycliques, notamment dans le degré d'eutrophisation de l'eau (milieu souvent mésotrophe) et l'extension des berges humides.

\section{LE SUBBORÉAL : NÉOLITHIQUE RÉCENT-ÂGE DU BRONZE}

Le Subboréal (UL3) se caractérise par une nette élévation du plan d'eau, qui atteint un premier maximum à la cote $343 \mathrm{~m} \mathrm{NGF}$, et par une extension de la zone inondée. Cette transgression favorise l'homogénéisation et la synchronisation du remplissage sédimentaire des deux cuvettes. Elle pourrait être la conséquence de l'oscillation climatique humide identifiée un peu partout en Europe durant cette période (Hughes et al., 2000), y compris dans le bassin-versant ligérien (Carcaud et al., 2002).

Les microfossiles non polliniques indiquent toutefois des fluctuations de la tranche et de la qualité des eaux, qui deviennent franchement eutrophes vers la fin de cette période (âge du Bronze). L'installation d'une nécropole [CO-081, fig. 15], datée du Bronze final 1, sur le delta oriental implique que le plan d'eau était alors situé plus bas, mais il est probable que ce secteur soit resté encore relativement humide. On sait que les morts étaient fréquemment relégués dans des zones insalubres à cette époque. 


\section{LE DÉBUT DU SUBATLANTIQUE : ÂGE DU FER-HAUT-EMPIRE}

Le début du Subatlantique est marqué par une augmentation de la tranche d'eau, suivie d'un abaissement du plan d'eau puis par l'assèchement de la dépression. Plusieurs indices permettent de préciser le rythme et les modalités de cette évolution :

- la netteté de la limite entre UL3 et la couche noire (CN) implique une phase d'assèchement antérieure à 403262 cal. B.C., âge le plus ancien de la couche noire obtenu sur S177, dans la zone deltaïque orientale ;

- le gonflement de la part des pollens de Cypéracées à la transition des biozones $h$ et $i$ traduit un gain de terres sur le marais ;

- la présence, sur le delta oriental, d'un habitat [CO-082, fig. 17 à 19] occupé de manière continue entre La Tène B1 $\left(\mathrm{IV}^{\mathrm{e}}\right.$ s. av. J.-C.) et La Tène D2 ( ${ }^{\mathrm{er}} \mathrm{s}$. av. J.-C.) indique que ce secteur était devenu habitable à cette époque ; l'existence de trois bâtiments gallo-romains [CO-084, CO-085, CO086, fig. 20] montre que c'est encore le cas durant les deux premiers siècles de notre ère ;

- plusieurs indices suggèrent même que la cuvette était complètement asséchée ou presque entre La Tène moyenne (III ${ }^{\mathrm{e}}$ s. av. J.-C.) et la fin du Haut-Empire (II ${ }^{\mathrm{e}}$ s. apr. J.-C.) :

- l'établissement [312, fig. 18 à 20], occupé entre La Tène moyenne et le milieu du $\mathrm{I}^{\mathrm{er}}$ s. apr. J.-C., qui est implanté dans la cuvette ;

- plusieurs zones funéraires datées des deux premiers siècles de notre ère, installées également au milieu de la cuvette [308, 309, 310, 311, 166, CO-012, CO-071, fig. 20] ;

- des épandages de mobilier datés entre le $\mathrm{I}^{\mathrm{er}} \mathrm{s}$. av. J.-C. et le $\mathrm{II}^{\mathrm{e}}$ s. apr. J.-C. (fig. 18 et 20 ) ;

- enfin, et surtout, le parcellaire borné que G. Vernet propose de dater au plus tard du début de la période augustéenne ;

- les données carpologiques suggèrent que, malgré la réduction du plan d'eau, le milieu était encore humide à la fin de l'âge du Fer, comme en témoigne la présence d'espèces inféodées aux bordures de marais, aux prairies humides et aux aulnaies; elles montrent la persistance de prairies humides durant le Haut-Empire, malgré le drainage.

L'augmentation de l'épaisseur de la tranche d'eau au début du Subatlantique pourrait correspondre à la phase d'humidification reconnue à cette époque (Van Geel et al., 1996), à laquelle J.-P. Daugas et al. (1983) imputaient le recul du peuplement dans la plaine de Limagne entre le
Bronze final et La Tène ancienne. Dans les plaines alluviales situées en amont de la Loire et dans ses affluents du bassin du Forez, une reprise de l'activité fluviale est observée à la charnière Bronze final/premier âge du Fer (Cubizolle, Georges, 2001 et 2002). On notera qu'à Sarliève, le delta oriental, qui avait été colonisé par la nécropole du Bronze final 1 [CO-081, fig. 15], n'est pas occupé durant le premier âge du Fer.

L'abaissement du plan d'eau au second âge du Fer et sa résorption aux environs du changement d'ère sontils la conséquence d'un climat moins humide ou l'effet d'entreprises de drainage conduites à proximité des centres de pouvoir? Les deux facteurs ont pu combiner leurs effets. On sait que les Arvernes acquirent précocement une excellente maîtrise technique dans le domaine hydraulique, qui leur permit de coloniser les marais de Limagne dès La Tène moyenne (Trément et al., 2004).

\section{LE SUBATLANTIQUE : BAS-EMPIRE-DÉBUT DE L'ÉPOQUE MODERNE}

La remise en eau du bassin s'effectue entre les III ${ }^{\mathrm{e}}-\mathrm{IV}^{\mathrm{e}} \mathrm{s}$. et le $\mathrm{X}^{\mathrm{e}} \mathrm{s}$., et la dépression fonctionne comme un véritable lac jusqu'à son assèchement artificiel au début du XVII ${ }^{\mathrm{e}} \mathrm{s}$. Les marqueurs de la remise en eau et de la formation du lac sont les suivants :

- le dépôt de la couche noire (CN), qui n'a pu s'opérer que sous une tranche d'eau, ce que confirme la présence de diatomées (Fleury, 2001). Or ce niveau scelle le comblement des fossés gallo-romains mis au jour par G. Vernet au cour de la cuvette. Les datations par le radiocarbone de ce niveau sont les suivantes : 403-262 cal. B.C. (dans S177), 94 cal. B.C.51 cal. A.D. (SP1), 68-220 cal. A.D. (SP1), 49 cal. B.C.222 cal. A.D. (SP4), 49 cal. B.C.-81 cal. A.D. (SARL 2A) ;

- la constitution du delta de seconde génération (US2), qui fossilise les vestiges gallo-romains du secteur de la Grande Halle, prouve que les eaux ont atteint, au moins temporairement, une cote supérieure à 346,50 m NGF, inondant les zones bordières entre les III ${ }^{\mathrm{e}}-\mathrm{IV}^{\mathrm{e}} \mathrm{s}$. et le début du XVII ${ }^{\mathrm{e}}$ s. ;

- même si l'argument a silentio est toujours critiquable, on ne peut qu'être frappé par l'absence totale de vestiges postérieurs au Haut-Empire dans la cuvette, alors que les indices évoqués plus haut sont si nombreux pour les siècles qui précèdent; or les rives, elles, continuent d'être densément occupées au Bas-Empire et au début du Moyen Âge ;

- la toponymie peut fournir deux indices supplémentaires en faveur d'une remise en eau précoce de la dépression. 
Deux toponymes aujourd'hui disparus marquent les extrémités septentrionale et méridionale de l'actuelle cuvette: Caplacus (954-986) et Sumlau (XI ${ }^{\mathrm{e}} \mathrm{s}$.). Contenant le radical latin lacus et relevant de types bien connus en Gaule ou en Italie (*Capu lacu, *Summu lacu), ces composés parallèles constituent un couple révélateur dont la formation peut être située, selon J.-P. Chambon, entre le $\mathrm{III}^{\mathrm{e}}$ et le $\mathrm{V}^{\mathrm{e}} \mathrm{s}$. (Chambon, Trément, 2004);

- les textes médiévaux et modernes mentionnent explicitement un lac en eau claire entre le $\mathrm{X}^{\mathrm{e}} \mathrm{s}$. et le début du

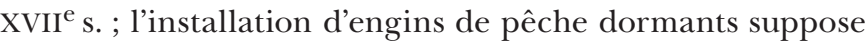
une profondeur d'eau relativement importante, qui nécessitait l'utilisation d'embarcations. Le niveau du lac était toutefois fluctuant;

- les microfossiles non polliniques pourraient rendre compte de ces fluctuations, indiquant une très forte eutrophisation des eaux et des phases à faible profondeur.

La remise en eau qui s'opère vraisemblablement dès le $\mathrm{III}^{\mathrm{e}} \mathrm{s}$. de notre ère est-elle la conséquence d'une fluctuation humide, dont l'existence est démontrée par ailleurs (Provansal et al., 1999), ou celle d'un changement du type de gestion du milieu ? Ces deux registres de causalité se sont vraisemblablement combinés dès la fin du Haut-Empire dans la moyenne vallée du Rhône (Berger, 1996 ; Berger, Jung, 1999 ; Berger et al., 2001).

Le maximum altimétrique absolu du plan d'eau est enregistré entre les $\mathrm{III}^{\mathrm{e}}-\mathrm{IV}^{\mathrm{e}} \mathrm{s}$. et le début du XVII ${ }^{\mathrm{e}} \mathrm{s}$. Il se traduit par la constitution d'un delta, qui témoigne d'événements pluviométriques très violents. Il est pour l'heure impossible de caler précisément cet épisode dans la chronologie ${ }^{7}$. À la fin du VI ${ }^{\mathrm{e}}$ s., Grégoire de Tours signale des inondations catastrophiques qui détruisent récoltes, troupeaux et maisons en Limagne (Historia Francorum, V, 25, 33). On sait qu'à partir de la fin de l'Antiquité et durant le haut Moyen Âge, le milieu subit de profonds changements en Grande Limagne (Ballut, 2000 ; Trément et al., 2002). Le système de drainage antique n'est apparemment plus efficace ou est même abandonné.

Enfin, les mentions récurrentes de débordements dans les textes du XVI ${ }^{\mathrm{e}}$ s. et $\mathrm{du} \mathrm{XVII}^{\mathrm{e}} \mathrm{s}$. pourraient être mises en rapport avec l'oscillation humide du Petit Âge Glaciaire.

\section{LA FIN DU SUBATLANTIQUE : XVII ${ }^{\mathrm{e}}-\mathrm{XXI}^{\mathrm{e}} \mathrm{S}$.}

Le paysage lacustre disparaît au début du XVII ${ }^{\mathrm{e}}$ s., suite à une entreprise de mise en valeur opérée par une famille de

7. Sur la «crise environnementale de la fin de l'Antiquité et du haut Moyen Âge », voir Leveau et al., 2000. promoteurs étrangers, les Strada, qui se concrétise par des remembrements et des travaux d'assèchement (Trément, 2004).

\section{GESTION DU MILIEU ET IMPACT DE L'OCGUPATION DU SOL}

À partir du Néolithique, les variations du plan d'eau sont étroitement corrélées avec les dynamiques de l'occupation du sol dans le bassin de Sarliève. On sait pourtant, à la lumière des recherches conduites récemment en Grande Limagne, que tout déterminisme simpliste est à proscrire (Trément et al., 2004). La localisation des implantations à distance du marais ne signifie pas que les zones humides n'étaient pas utilisées comme pâturages ou pour leurs ressources spécifiques (végétaux, argile, gibier d'eau, poissons, éventuellement sel).

\section{LE NÉOLITHIQUE}

Les plus anciens indices d'agriculture reconnus dans le bassin de Sarliève sont légèrement postérieurs à 59755637 cal. B.C., ce qui les place, logiquement, dans le courant du Néolithique ancien. Il s'agit de grains de pollen de céréales reconnus vers la base de la carotte SARL 2B (biozone b) en association avec des indices de défrichage. Cette période est pour ainsi dire inconnue en Auvergne. Elle se caractériserait dans la plaine par une faible densité de population et par un développement précaire des techniques agricoles (Delpuech, 1987, p. 26). Les deux tessons de céramique à pastillage recueillis en prospection sur les sites [264] et [274] (fig. 12 et 13) confirment la réalité de cette occupation dans le bassin de Sarliève à la fin du Néolithique ancien.

Les indices d'activité agricole deviennent plus nets dans le courant de l'Atlantique, témoignant de l'ouverture de clairières aux dépens de la forêt de chênes, de l'extension des cultures et de la création de pâturages (SARL 2B.c1). Les champignons typiques du début de la dégradation de la forêt, à écologie coprophile, traduisent l'augmentation des populations animales autour du marais. Les bouffées de pollen tertiaire signent une intensification de l'érosion sur les versants. L'étude sédimentologique du remplissage des cuvettes confirme une augmentation épisodique de l'érosion mécanique, notamment dans les couches interlamines de l'unité lacustre UL2, alors que l'érosion chimique dominait largement auparavant. On peut mettre cette évolution en rapport avec la densification des installations 
durant le Néolithique moyen. Celle-ci reflète une appropriation véritable du territoire par les communautés agropastorales chasséennes. L'habitat du Néolithique moyen, qui se concentre majoritairement sur les plateaux, les versants et les piémonts, a pu bénéficier des importantes fluctuations du plan d'eau qui caractérisent l'Atlantique pour coloniser, au moins temporairement, la zone de l'émissaire et la bordure orientale.

Palynologie et étude des microfossiles non polliniques pourraient traduire les effets d'une gestion itinérante et cyclique du milieu par des communautés plus ou moins nombreuses mais mobiles, susceptibles d'avoir localement un fort impact sur l'environnement, du fait d'une gestion peu économe des sols et d'une implantation privilégiant les zones de versants, particulièrement sensibles à l'érosion, sur lesquels elles pratiquent déboisements, brûlis et pâturage. Dans la biozone SARL 2B.c2, les microfossiles non polliniques indiquent une intensification de la déforestation concomitante d'incendies à caractère régional, favorisés peut-être par une relative sécheresse. Or ces phases caractérisées par l'utilisation du feu sont précédées par un gonflement des marqueurs de l'élevage, en particulier des champignons coprophiles, et sont contemporaines d'un développement des cultures céréalières. Le recours à cette pratique était le seul moyen d'étendre les cultures et les pacages (Sigaut, 1975 ; Berger, Thiébault, 2000).

La fin de l'Atlantique est marquée par un climat plus frais et plus humide, propice au développement du hêtre, du sapin et du chêne, ainsi qu'à la montée du plan d'eau. Ces tendances s'accentuent au Subboréal, dont la première partie correspond au Néolithique récent/final. La tranche d'eau s'épaissit alors fortement. Le développement de la couverture forestière sur les versants les protège mieux contre l'érosion mécanique : on observe en effet, dans les sédiments, une reprise de l'érosion chimique, même si les apports par érosion mécanique restent prédominants. L'impact des communautés agropastorales sur le milieu est cependant bien perceptible au cours de cette période, sous la forme d'une brusque augmentation des pollens de céréales et de rudérales, d'une régression du noisetier, d'une intensification de l'érosion mécanique et d'une accentuation de l'eutrophisation des eaux.

\section{L'ÂGE DU BRONZE}

L'âge du Bronze est marqué par une évolution complexe, dont il est impossible de préciser la chronologie. La désertion des zones basses et des piémonts, probablement dès le Néolithique récent/final, pourrait s'expliquer par la nette élévation du plan d'eau au Subboréal. On peut supposer que celle-ci a provoqué la désorganisation du drainage sur les bordures du marais et dans les vallons affluents, contribuant à rendre insalubres les zones basses. Le diagramme d'anthropisation végétale fait apparaître une succession de cycles d'expansion agricole et de reprise forestière corrélés à une accentuation de l'érosion mécanique sur les versants. La forêt est majoritairement composée de chênes, de hêtres et de sapins. La hêtraie, qui atteint son développement maximal, fait l'objet d'une exploitation. Les clairières ainsi ouvertes sont vouées au pâturage. Les cultures de céréales sont attestées pendant toute la période, mais moins nettement qu'au Néolithique. Le noyer fait son apparition.

Il est impossible de corréler précisément les pulsations du système agricole et les cartes archéologiques. Ces dernières mettent en évidence deux phases de peuplement relativement dense, au Bronze ancien et au Bronze final, séparées par une apparente rétraction au Bronze moyen. L'habitat, qui se concentre sur les versants et les hauteurs durant le Bronze ancien et moyen, amorce une timide descente au Bronze final. Ses dynamiques spatiales contrastées renvoient, comme les données paléoenvironnementales, à des cycles d'exploitation du milieu dans lesquels ont dû interférer les importantes fluctuations du plan d'eau.

\section{L'ÂGE DU FER}

Le début du Subatlantique est caractérisé par une nette accentuation de la pression anthropique sur le milieu, qui se traduit par l'amorce d'un lent déclin de la chênaie-hêtraie, par l'extension des pâturages et des prairies humides à la faveur du déboisement des rives, par une expansion des cultures et de l'élevage, enfin, par un accroissement de l'eutrophisation des eaux.

Le rythme de cette évolution n'est apparemment pas continu. Deux phases de pression agricole sont marquées dans la biozone SARL 2B.h :

- la première pourrait correspondre à la densification de l'habitat au premier âge du Fer ;

- la seconde, qui se caractérise par une accentuation des tendances précédentes et par un renouveau de la pratique du brûlis, pourrait prendre place dans le contexte d'expansion démographique de La Tène finale.

$\mathrm{Au}$ Bronze final et au premier âge du Fer, les oscillations du plan d'eau interfèrent de manière complexe avec les effets, sur l'habitat, des mutations politiques et socio- 
économiques. L'installation d'une nécropole [CO-081, fig. 15] sur l'ancien delta oriental au Bronze final 1 ne signifie pas forcément que le lieu était salubre. Au premier âge du Fer, l'occupation des zones basses s'avère encore bien timide, même si la bordure du marais n'apparaît pas franchement répulsive. Faut-il voir là la conséquence d'une remontée du plan d'eau suggérée par les microfossiles non polliniques au début du Subatlantique?

Le comportement de l'habitat vis-à-vis du marais évolue considérablement au second âge du Fer, sous l'effet de l'abaissement progressif du plan d'eau et d'une probable expansion démographique qui rend nécessaire la mise en valeur de nouvelles terres. Dès La Tène ancienne, la zone de l'ancien delta oriental est occupée par un établissement pérenne associé à une nécropole [CO-082, CO-083, fig. 17]. On peut supposer que ses occupants ont cherché à s'implanter le plus près possible de la cuvette, dans une zone légèrement dominante et mieux drainée que les autres points de la bordure du marais, du fait du sous-sol sableux. À La Tène moyenne, l'installation d'un établissement [312, fig. 18] dans la cuvette septentrionale implique que le plan d'eau y était déjà largement résorbé. Cette tendance s'accentue à La Tène finale, avec la colonisation de la bordure orientale. Dans le bassin sud, en revanche, l'absence de toute implantation dans la cuvette pourrait indiquer que celle-ci demeurait partiellement en eau. La nette densification de l'habitat sur la terrasse orientale, qui se traduit par le développement d'un véritable chapelet d'établissements dominant le fond du marais, prouve que les terres bordières étaient mieux drainées qu'auparavant.

La palynologie montre clairement que de nouvelles terres sont gagnées sur le marais au cours de l'âge du Fer. Les déboisements opérés sur les rives aux dépens de la chênaie-hêtraie s'accompagnent d'une extension des pâturages et des prairies humides. La carpologie révèle l'existence, à côté des champs de céréales, de cultures sarclées enrichies en nitrate par rejet de substances riches en azote ou ajout d'engrais organiques (présence de plantes nitrophiles comme l'arroche hastée, le chénopode blanc et l'ortie). L'étude des microfossiles non polliniques confirme l'intensification du pastoralisme sur la bordure du marais. Les analyses archéozoologiques conduites par P. Caillat sur l'habitat de la Grande Halle [CO-082, fig. 18] suggèrent que le cheptel se composait de bovidés, d'ovinés et de suidés, sans permettre d'en savoir plus sur la part respective de chaque espèce.

L'étude paléopathologique menée par M. Billard sur les squelettes de la nécropole laténienne [CO-083, fig. 18] révèle que l'état sanitaire misérable d'une partie de la population qui occupait le delta oriental pourrait être lié non seulement à des carences alimentaires, mais aussi à la promiscuité avec le bétail et à la proximité de zones palustres (hyperostose porotique, atteintes tuberculeuses et peut-être brucelliennes). La carpologie indique d'ailleurs clairement que la bordure du marais demeurait très humide.

Il apparaît, à la lumière des données fournies par la carpologie et par la palynologie, que cette période est caractérisée par un paysage très ouvert, dominé par les cultures et les prairies, où les bois et les fourrés sont rares. La diversification des espèces cultivées et la recherche d'une certaine intensification témoignent d'une bonne maîtrise agricole, visant à satisfaire les besoins d'une population croissante. Les prospections révèlent en effet, pour La Tène finale, une densité de peuplement jusqu'alors inégalée. L'installation d'établissements nombreux dans tous les types de milieux a dû nécessiter l'usage du feu pour le défrichage. Malheureusement, du fait de l'exondation, l'impact érosif de cette expansion agricole n'a pas été enregistré dans la cuvette.

\section{L'ÉPOQUE ROMAINE}

Pour la même raison, l'époque romaine est mal documentée du point de vue paléoenvironnemental. Les échantillons polliniques prélevés à la base du fossé F168 révèlent un paysage très ouvert, dans lequel subsistent quelques îlots forestiers. Cette image est conforme à celle obtenue par L. Gachon dans les niveaux gallo-romains de la villa de Belde [336, fig. 20], à quelques centaines de mètres au nord de la cuvette (Gachon, 1963, p. 143 et tabl. 20). La carpologie témoigne d'une diversification des plantes cultivées dès l'époque augustéenne. La carte archéologique du HautEmpire confirme la généralisation du système agricole à l'ensemble du bassin-versant, dans le cadre de l'économie domaniale, dans un contexte de forte pression démographique qui s'explique par la proximité du chef-lieu de cité Augustonemetum.

Le plan d'eau n'est pas complètement résorbé avant la fin de La Tène finale ou l'époque augustéenne. C'est à ce moment qu'est mis en place le parcellaire borné identifié au milieu de la cuvette septentrionale. Dès le $\mathrm{I}^{\mathrm{er}} \mathrm{s}$. de notre ère, plusieurs établissements s'implantent dans la moitié est de la cuvette septentrionale, tandis que des nécropoles à incinération se localisent dans les points les plus bas, tant au nord qu'au centre et au sud de la dépression. La distribution des épandages agraires laisse supposer que tout le fond de la cuvette était cultivé. On pense à des prairies de fauche 
ou à des pâturages appartenant aux grands domaines qui jalonnaient la bordure de l'ancien marais asséché.

Le dépôt de la couche noire charbonneuse, dès la fin du Haut-Empire, correspond à un changement dans le type de gestion du milieu, qui se traduit par l'abandon des fossés de drainage, la remise en eau de la cuvette et la pratique généralisée des brûlis. L'épaisseur, l'étendue et la cohérence de cette couche attestent l'ampleur du phénomène. Faut-il voir là la conséquence d'un rééquilibrage de l'économie au profit de l'élevage? Grégoire de Tours (Liber de passione et virtutibus sancti Juliani, 17) évoque bien un pastoralisme transhumant entre la vallée de l'Allier et les plateaux d'altitude au $\mathrm{VI}^{\mathrm{e}} \mathrm{s}$., mais on ne sait pas si ce phénomène concernait le bassin de Clermont. Dans la moyenne vallée du Rhône, une "phase de paléoincendies généralisée " a été mise en évidence par J.-F. Berger (2001, p. 393) «à la transition entre le Bas-Empire et l'Antiquité tardive (vers 1600-1500 B.P., soit un pic de probabilité situé autour du $\mathrm{VI}^{\mathrm{e}}$ s. apr. J.-C. en données calibrées) ». L'auteur est tenté d'imputer ces feux aux pratiques agropastorales de la fin de l'Antiquité, caractérisées par une exploitation des points hauts et par un développement du pastoralisme dans les piémonts et les lits majeurs des cours d'eau ${ }^{8}$.

La couche noire implique une remise en eau du marais, postérieure au $\mathrm{II}^{\mathrm{e}}$ s., qui s'accompagne d'un retrait définitif de l'habitat du fond de la cuvette. Les rives du marais ne sont pas pour autant désertées. Les exploitations qui s'y étaient développées au Haut-Empire perdurent quasiment toutes au Bas-Empire. Malgré la formation d'un véritable lac, une partie de ces établissements est encore occupée durant le haut Moyen Âge, voire au bas Moyen Âge. À l'image de ce qui a été observé dans le Grand Marais, à quelques kilomètres au nord, il s'agit là d'un bel exemple de résilience du réseau de l'habitat antique, dans un contexte environnemental pourtant profondément modifié (Trément et al., 2004).

\section{DE LA FIN DE L’ANTIQUITÉ AU MOYEN ÂGE}

La mise en place de la séquence sédimentaire UL4 superposée au niveau noir ne peut pas avoir débuté avant le Bas-Empire. Elle est marquée par l'effondrement des pollens d'arbres (SARL 2B.i), qui reflète le déclin consommé de la chênaie-hêtraie, et par le développement important des cultures et des étendues herbeuses liées au substrat humide.

8. Sur la pratique des feux agropastoraux à la fin de l'Antiquité et durant le haut Moyen Âge, voir la synthèse de A. Durand et P. Leveau, 2004, p. 201-204.
Dans le sondage SP4, la forte représentation des pollens tertiaires est l'indice d'une érosion mécanique active qui se traduit par l'arrivée massive de sédiments dans la zone deltaïque orientale. La sédimentologie confirme l'intensification de l'érosion mécanique sur les versants. Les dépôts deltaïques grossiers de la bordure orientale témoignent de la forte intensité et du pouvoir érosif de certains événements pluviométriques. Les microfossiles non polliniques indiquent une continuité des activités pastorales, l'utilisation du feu comme moyen de déforestation et une accentuation de l'eutrophisation du plan d'eau, dont la profondeur paraît alors assez peu importante.

À partir de l'an mil, les textes montrent que le lac contribue à former un paysage original et complexe, où la limite entre eaux libres et terrains marécageux est fluctuante. Les nombreux partages dont il fait l'objet entre paroisses, seigneuries et domaines ecclésiastiques prouvent qu'il est un enjeu économique de premier ordre. Il procure en effet des ressources variées : produit de la pêche, exploitation de la roselière, pacages et herbages. De nouveaux espaces sont gagnés sur ses marges pour l'élevage et même les cultures. Ce système d'exploitation traditionnelle disparaît au début du XVII ${ }^{\mathrm{e}}$ s., au profit d'une mise en valeur globale qui nécessite assèchements et remembrements.

F. T.

$$
\text { * * }
$$

Durant plus d'un demi-millénaire, du $\mathrm{X}^{\mathrm{e}} \mathrm{s}$. au début du $\mathrm{XVII}^{\mathrm{e}}$ s., le nom de Sarliève a évoqué pour les contemporains une cuvette marécageuse et lacustre, étroitement intégrée à l'économie locale au profit des seigneuries et communautés villageoises installées sur ses rives, mais aussi au profit d'établissements religieux qui y trouvaient des ressources complémentaires. Le bassin-versant s'inscrit dans les représentations mentales comme une unité paysagère cohérente, centrée sur le marais.

Pourtant, l'un des apports des recherches conduites depuis 1997 est d'avoir mis en lumière la grande variabilité du milieu humide à l'Holocène. Cette variabilité est telle qu'elle oblige à utiliser différents termes, suivant les périodes, pour qualifier la même unité physique : marais, lac, paléolac, cuvette ou dépression. Il apparaît que le plan d'eau fluctue selon des cycles régis par plusieurs temporalités. L'alternance de phases de mise en eau et d'assèchement est la situation la plus courante, même lorsque la cuvette fonctionne comme un véritable lac (au Moyen Âge et peut-être au Subboréal) ; à l'inverse, il est vraisemblable que, malgré le drainage antique, le fond de la dépression 
soit toujours resté assez humide. Ce qui change, dans la longue durée, c'est l'ampleur des oscillations verticales du plan d'eau, conditionnées par le climat mais également par le niveau de base de l'exutoire et par les aménagements humains. Constitué de coulées boueuses, le niveau de base de l'exutoire a pu lui-même varier sous l'inflence de facteurs naturels ou anthropiques.

Un autre apport de cette étude réside dans la mise en évidence des relations entre fluctuations du plan d'eau et occupation des rives. Si aucun déterminisme physique ne préside aux mouvements de l'habitat, il est clair que le milieu humide a pu présenter, par moments, une contrainte forte pour le peuplement et pour les cultures. Avant la stabilisation de l'habitat et du système agricole, à l'âge du Fer, la forte mobilité des implantations permettait aux communautés de s'accomoder des variations du milieu humide. Les groupes humains ont assurément tiré le meilleur profit de la complémentarité des ressources fournies par un milieu aussi diversifié : versants et plateaux aux sols légers et bien drainés, biodiversité du marais, terrains de pacage sur ses bordures. Il est probable, d'ailleurs, que certaines implantations reconnues dans des zones basses soumises à l'humidité n'aient été occupées que par intermittence, saisonnièrement. Autre exemple d'adaptation aux conditions du milieu : lorsque l'abaissement du plan d'eau à la fin de l'âge du Bronze puis dans le courant de l'âge du Fer est propice à la colonisation des bas-fonds, les secteurs les moins salubres sont d'abord voués aux morts avant de l'être aux vivants.

À la fin de l'âge du Fer et durant le Haut-Empire, l'assèchement de la dépression nécessita la réalisation d'impor- tants travaux, dont plusieurs fossés bornés ont conservé la trace. La dernière étendue d'eau subsistant au pied de l'oppidum de Gergovie et aux portes d'Augustonemetum était résorbée, au profit, vraisemblablement, de gros propriétaires fonciers dont les villae bordaient l'ancien marais. À partir de ce moment, et quelle que soit l'ampleur des fluctuations du plan d'eau, on observe, comme dans le reste de la Limagne, une grande stabilité du réseau des établissements agricoles.

Cette première synthèse confirme la pertinence du choix du bassin-versant pour appréhender dans la longue durée l'histoire des interactions sociétés-milieux. Le bassinversant est un système au sein duquel interagissent en permanence variables biophysiques, climatiques et anthropiques. C'est à cette échelle qu'une compréhension globale des processus est possible.

De toute évidence, les caractéristiques intrinsèques du bassin de Sarliève mais aussi sa localisation au cœur du territoire des Arvernes en font un excellent enregistreur des dynamiques environnementales, mais aussi sociétales. Nos recherches ont montré qu'une vraie démarche interdisciplinaire est à même d'assurer, à cette échelle spatiale, le décryptage des informations contenues dans le sous-sol, à sa surface ou dans les archives écrites. Bien des questions subsistent toutefois. Il est maintenant nécessaire d'affiner la résolution temporelle des données paléoenvironnementales. À la lumière des premiers résultats et du schéma d'interprétation proposé, un retour sur le terrain s'impose, avec un questionnement fondamentalement renouvelé.

F. T. 


\section{BIBLIOGRAPHIE}

\section{ARGANT J.}

1990 : Climat et environnement au Quaternaire dans le bassin du Rhône d'après les données palynologiques, Villeurbanne, Université Claude-Bernard et Département des sciences de la terre (coll. Documents des Laboratoires de géologie de Lyon, 111), $199 \mathrm{p}$.

\section{BALlUT C.}

2000: Évolution environnementale de la Limagne de Clermont-Ferrand au cours de la seconde moitié de l'Holocène (Massif central français), thèse de Doctorat, Université de Clermont-Ferrand, 315 p. [inédit].

Beaulieu J.-L. De, Pons A., ReIlle M.

1988: " Histoire de la flore et de la végétation du Massif central (France) depuis la fin de la dernière glaciation ", Cahiers de micropaléontologie, 3, p. 5-36.

BERGER J.-F.

1996: "Climat et dynamique des agrosystèmes dans la moyenne vallée du Rhône", in FICHES J.-L. (DIR.), Le III siècle en Gaule Narbonnaise: données régionales sur la crise de l'Empire, Sophia Antipolis, éd. APDCA, p. 299-332.

\section{BERGER J.-F., JUNG C.}

1999: «Developing a methodological approach to the evolution of field systems in the Middle Rhône valley", in Leveau P., Trément F., Walsh K., BARKER G. (DIR.), Environmental Reconstruction in Mediterranean Landscape Archaeology, Oxford, Oxbow Books (coll. The Archaeology of Mediterranean Landscape, 2), p. 155-167.

BERger J.-F. AVEC LA COLlab. DE JUNG C., THIÉBAULT S.

2001 : «Évolution des agro- et des hydrosystèmes dans la région médio-rhodanienne ", in Ouzoulias P., Pellecuer C., RAYNAUD C., VAN OSSEL P., GARMY P. (DIR.), Les Campagnes de la Gaule à la fin de l'Antiquité, Antibes, éd. APDCA, p. 369-403.

\section{BERGER J.-F., THIÉBAULT S.}

2000 : « Holocene fire history in South East France: impact of climatic changes and human activities ", in SCOTT A. C., MORE J., BRAYShaY N. B. (DIR.), Fire and the Palaeoenvironment, Actes du colloque de
Sheffield, 12-13 avril 1997, Amsterdam, Elsevier (coll. Palaeogeography, Paleoclimatology, Paleoecology Special Issue, 164), $410 \mathrm{p}$.

\section{BICHET V., CAMPY M., BUONCRISTIANI J.-F.,} Di GIOVANNI C., MEYBECK M.,

\section{RICHARD H.}

1999 : «Variations in sediment yield from the Upper Doubs River carbonate watershed (Jura, France) since the Late-Glacial Period ", Quaternary Research, 51, p. 267279.

\section{BOUBY L.}

1993: Apports de la carpologie à la connaissance de l'économie végétale de l'Auvergne $d u$ Mésolithique à lépoque gallo-romaine, mémoire de DEA, Université MontpellierII, 110 p. [inédit].

Bournérias M., ARNAl G., Bock C. 2001 (4 $\left.\mathrm{e}^{\mathrm{e}} \mathrm{d}.\right)$ : Guide des groupements végétaux de la région parisienne, Paris, Belin, $639 \mathrm{p}$.

Bréhéret J.-G., MACAIre J.-J., FleUry A., FOURMONT A., SOULIÉ-MÄRSCHE I.

2003: "Indices de confinement dans les dépôts lacustres holocènes de Sarliève (Limagne, France) ", in Comptes rendus de l'Académie des sciences, 335, p. 479-485.

\section{Cabanis M.}

2002: Étude carpologique du site de Sarliève en Limagne (Puy-de-Dôme) ou les pratiques culturales durant le deuxième âge du Fer et l'époque gallo-romaine, mémoire de DEA, Université Paris-I, 89 p.

\section{Cabézuelo U.}

2001: "Domaine de Sarliève (Cournon)", Bilan scientifique du Service régional de l'archéologie, Auvergne, Ministère de la Culture, Direction régionale des Affaires culturelles d'Auvergne, p. 98.

\section{Garcaud N., Garcin M., VisSet L.,} MUSCH J., BURNOUF J.

2002: "Nouvelle lecture de l'évolution des paysages fluviaux à l'Holocène dans le bassin de la Loire moyenne", in BRAVARD J.-P., MAGNY M. (DIR.), Les Fleuves ont une histoire : paléo-environnement des rivières et des lacs français depuis 15000 ans, Paris, éd. Errance (coll. Archéologie aujourd'hui), p. 71-84.
Chambon J.-P., TRÉment F.

2004: «Un couple de toponymes référant à un lac disparu près de Clermont-Ferrand (Auvergne) : *Summu lacu, *Capu lacu: confrontation des données linguistiques, archéologiques et paléoenvironnementales ", Zeitschrift für romanische Philologie, 120 , 2, p. 266-281.

\section{Cubizolle H., GeORges V.}

2001: "L'évolution holocène de la plaine alluviale de la Loire dans le bassin du Forez (Massif central, France) : mise en évidence du potentiel archéologique", Quaternaire, 12, 1-2, p. 53-67.

Cubizolle H., GeOrges V. avec la collab. de Argant J., Petit F.

2002: «Évolution morphosédimentaire des plaines alluviales de la Loire et de ses affluents dans le bassin du Forez (Massif central français) depuis la fin du Würm », in BRAVARD J.-P., MAGNYM. (DIR.), Les Fleuves ont une histoire : paléo-environnement des rivières et des lacs français depuis 15000 ans, Paris, éd. Errance (coll. Archéologie aujourd'hui), p. 63-70.

DaUgas J.-P., Loison G., Vital J.

1983: "Lâge du Bronze en Auvergne", in Les Inédits de la Préhistoire auvergnate, Clermont-Ferrand, Musée Bargoin, $271 \mathrm{p}$.

\section{DELPUECH A.}

1987: Deux millions d'années en Auvergne: archéologie et autoroute A71, Société des autoroutes Paris-Rhin-Rhône, Scetauroute, Direction régionale des Affaires culturelles d'Auvergne, Direction des Antiquités, Marsat, $62 \mathrm{p}$.

\section{DERRUAU M.}

1949 : La Morphogenèse de la Grande Limagne et ses conséquences sur la morphologie des plateaux bordiers, thèse de Doctorat complémentaire, Université de Grenoble, 181 p. [inédit].

Dousteyssier B., Segard M., TRÉment F. 2004: "Les villae gallo-romaines dans le territoire proche d'Augustonemetum (Clermont-Ferrand) : approche critique de la documentation archéologique", Revue archéologique du Centre de la France, 43, p. 115-147. 
DURAND A., LEVEAU P.

2004: «Farming in Mediterranean France and rural settlement in the Late Roman and Early Medieval periods: the contribution from archaeology and environmental sciences in the last twenty years (1980-2000) », in BARCELÓ M., SIGAUT F. (DIR.), The Making of Feudal Agricultures?, Boston-Leiden, Brill, p. 177-253.

\section{EINSELE G., HINDERER M.}

1998: "Quantifying denudation and sediment-accumulation systems (open and closed lakes) : basic concepts and first results », Palaeogeography, Palaeoclimatology, Palaeoecology, 140, p. 7-21.

\section{ELLENBERG H.}

1988: Vegetation Ecology of Central Europe, Cambridge, New York, New Rochelle, Melbourne, Sydney, Cambridge University Press, 731 p.

FICHES J.-L. (DIR.)

1996: Le III siècle en Gaule Narbonnaise: données régionales sur la crise de l'Empire, Sophia Antipolis, éd. APDCA, 404 p.

\section{FLEURY A.}

2001: Apport des diatomées à la caractérisation de l'environnement lacustre du marais de Sarliève (Puy-de-Dôme) au cours de l'Holocène et de la période historique, mémoire de DEA, Université Orléans-Tours, 38 p. et annexes.

\section{FOURMONT A.}

2005: Érosion et sédimentation dans le bassin de Sarliève (Massif central, France) au Tardiglaciaire et à l'Holocène: impact des facteurs naturels et anthropiques, thèse de Doctorat, Université de Tours, 420 p. [inédit].

FOURMONT A., MACAIRE J.J., BÉHÉRET J.-G., Argant J., Prat B., Vernet G.

2006: "Tephras in lacustrine sediments of the Sarliève marsh (French Massif central) : age and preservation ", Comptes rendus Géosciences, 338, p. 1141-1149.

\section{FOURNIER G.}

1996: "Sarliève: un lac au Moyen Âge", Bulletin de l'Association du site de Gergovie, 11 , p. 2-34.

2000 : "Le lac de Sarliève au Moyen Âge ", Revue d'Auvergne, 554-555, n ${ }^{\circ} 1 / 2$, t. 114, p. 128-130.

\section{FOURVEL A., LIEGARD S.}

1996: Document final de synthèse de sauvetage urgent de 1993 du site de Maréchal à
Romagnat (Puy-de-Dôme), Service régional de l'archéologie, Clermont-Ferrand.

\section{GACHON L.}

1963: Contribution à l'étude du Quaternaire récent de la Grande Limagne marno-calcaire : morphogenèse et pédogenèse, Annales agronomiques, vol. 14, $\mathrm{n}^{\circ}$ hors-série I, $191 \mathrm{p}$.

\section{GaY I., MACAIRE J.-J., Cocirta C.}

1998 : «Évolution qualitative des flux particulaires depuis 12600 ans dans le bassin du lac Chambon, Massif central, France ", Bulletin de la Société géologique de France, 169, p. 301-314.

\section{HAVINGA A. J.}

1971 : «An experimental investigation into the decay of pollen and spores in various soil types ", in BROOKS J., GRANT P. R., MUIR M. D., VAN GIJZEL P., SHAW G. (DIR.), Sporopollenin, London, New York, Academic Press, p. 446-479.

1984 : «A 20 year experimental investigation into the differential corrosion susceptibility of pollen and spores in various soil types ", Pollen et spores, XXVI, 3-4, p. $541-557$.

HUGUES P. D. M., MAUQUOY D.,

BARBER K. E., LANGDON P. G.

$2000:$ : Mire development pathwaysand paleoclimatic records from a full Holocene peat archive at Walton Moss, Cumbria, England ", Holocene, 10, p. 465-479.

JEAMBRUN M., GIOT D., BOUILLER R., BAUdRY D., CAMUS G., GUYONNAUd G., WEECKSTEEN M. G., LEROY B.

1973: Carte géologique à 1:50 000 de ClermontFerrand, XXV-31, Bureau de recherches géologiques et minières.

JEAN-BLAIN C.

1973 : Plantes vénéneuses : toxicologie, Paris, La Maison rustique, $139 \mathrm{p}$.

JULLIAN C.

1901 : Vercingétorix, Paris, Hachette, 406 p.

\section{KROONENBERG S. B., VAN DEN BERG} VAN SAPAROEA R. M., JONKE A. T. J.

1987 : «Late Glacial and Holocene development of semi-closed depressions (thaw lakes ?) in the Limagne Rift Valley, French Central Massif ", Geologie en Mijnbouw, 66, p. $297-311$.

\section{LENSELINK G., KROONENBERG S. B.,} LOISON G.

1990 : «Pleniglacial to Holocene paleo-environments in the Artière basin in the western Limagne rift valley, Massif central, France ", Quaternaire, 2, p. 139-156.

Leveau P., Provansal M., Bruneton H., Palet-Martinez J.-M., Poupet P., WALSH K.

2000: «La crise environnementale de la fin de l'Antiquité et du haut Moyen Âge : définition d'un modèle et retour aux milieux réels », in RICHARD H., VIGNOT A. (DIR.), Équilibres et ruptures dans les écosystèmes depuis 20000 ans en Europe de l'Ouest, Actes du colloque international de Besançon, 18-22 sept. 2000, Besançon (coll. Presses universitaires franc-comtoises, 831), p. 291-303.

\section{LOISON G.}

2003: LẦge du Bronze ancien en Auvergne, Toulouse, École des hautes études en sciences sociales (coll. Archives d'écologie préhistorique, 14), $156 \mathrm{p}$.

LOison G., COllis J., GUichard V., BENSIHAMED L., MARCHIANTI D., Pelissier E.

1991 : «Les pratiques funéraires en Auvergne à la fin du second âge du Fer : nouvelles données ", Revue archéologique du Centre de la France, 30, p. 97-111.

López Sáez J. A, VAN Geel B., FarbosTEXIER S., DIOT M.-F.

1998: "Remarques paléoécologiques à propos de quelques palynomorphes nonpolliniques provenant de sédiments quaternaires en France ", Revue de paléobiologie, 17, 2, p. 445-459.

MACAIRE J.-J., BELLEMLIH S., DI GIOVANNI C., DE LUCA P., VisSET L., BERNARD J.

2002: « Sediment yield and storage variations in the Négron river catchment (South Western Parisian Basin, France) during the Holocene Period", Earth Surface Processes and Landforms, 27, p. 991-1009.

Macaire J.-J., Bossuet G., Choquier A., CocirTa C., DE LUCA P., DUPIS A., GAY I., MATHEY E., GUENET P.

1997 : « Sediment yield during Late Glacial and Holocene periods in the lac Chambon watershed, Massif central, France ", Earth Surface Processes and Landforms, 22, p. 473489.

\section{MARGUERIE D.}

1992: Évolution de la végétation sous limpact humain en Armorique du Néolithique aux périodes historiques, Rennes, Université de Rennes (coll. Travaux du laboratoire d'anthropologie de Rennes, 40), 313 p. 


\section{MARINVAL P.}

1988: Cueillette, agriculture et alimentation végétale de l'Épipaléolithique jusqu'au deuxième âge du Fer en France méridionale: apports palethnographiques de la carpologie -1-Texte, thèse de Doctorat, Paris, École des hautes études en sciences sociales, 306 p. [inédit].

\section{MATHIEU P.-P.}

1862: Vercingétorix et César à Gergovia chez les Arvernes: mémoire où sont exposées et résolues, d'après de récentes découvertes, les questions relatives à cet épisode de l'histoire d'Auvergne, Clermont-Ferrand, Thibaud, $134 \mathrm{p}$

MENNESSIER-JOUANNET C. (DIR.)

1999-2002: Projet collectif de recherche sur les mobiliers du second âge du Fer en Auvergne : rapport annuel, Mirefleurs, Association pour la recherche sur l'âge du Fer en Auvergne.

\section{Mennessier-Jouannet C., Collis J.,} GUICHARD V.

2002: "L'occupation du sol en Limagne d'Auvergne de la fin du premier âge du Fer à la conquête romaine ", in MARTIN D. (DIR.), L'Identité de l'Auvergne (Auvergne-Bourbonnais-Velay), mythe ou réalité historique? : essai sur une histoire de l'Auvergne des origines à nos jours, Nonette, éd. Créer, p. 71-77.

Miallier D., Sanzelle S., Pilleyre T., VERNET G., BRUGIÈre S., DANHARA T.

2004: «Nouvelles données sur le téphra de Sarliève et le téphra CF7, marqueurs chronostratigraphiques de Grande Limagne (Massif central, France) ", in Comptes rendus de l'Académie des sciences, 336 , p. 1-8.

\section{MILCENT P.-Y.}

2002: "L'Auvergne à l'âge du Bronze ", in MARTIN D. (DIR.), L'Identité de l'Auvergne (Auvergne-Bourbonnais-Velay), mythe ou réalité historique?: essai sur une histoire de l'Auvergne des origines à nos jours, Nonette, éd. Créer, p. 65-70

2004: Le Premier âge du Fer en France centrale, Paris, Société préhistorique française (coll. Mémoires de la Société préhistorique française, XXXIV), 2 vol.

Miras Y., GUENET P., SURMEly F., Michelin Y., Tible R., WALTER-

SIMONNET A.-V., RICHARD H.

2003: «Histoire de l'environnement et des dynamiques agro-pastorales dans le massif du Cantal: la tourbière de
Roussy (Saint-Projet-de-Salers, France) », Quaternaire, 14, 4, p. 265-278.

\section{POMEL M.}

1853: « Catalogue méthodique et descriptif des vertébrés fossiles découverts dans le bassin hydrographique supérieur de la Loire et surtout dans la vallée de son affluent principal, l'Allier ", Annales scientifiques, littéraires et industrielles de l'Auvergne, XXVI, p. 81-229.

\section{POMMEROL F.}

1877: «Existence de l'homme en Auvergne à l'époque du renne et des volcans à cratère", in Comptes rendus de l'Association française pour l'avancement des sciences, $5^{\mathrm{e}}$ session, $11^{\mathrm{e}}$ section: Anthropologie, séance du 24 août 1876, Paris, p. 661668.

Pons A., Reille M., BeAulieU J.-L. DE, DELIBRIAS G., EVIN J.

1988: «Relations entre datations ${ }^{14} \mathrm{C}$ et analyse pollinique: l'exemple de l'intervalle 6000-4000 BP dans le Massif central ", Bulletin de l'Association française pour l'étude du Quaternaire, 1, p. 35-42.

PRAT B.

2006 : Systèmes agropastoraux et milieux périurbains en Basse-Auvergne au cours des trois derniers millénaires: contribution de l'analyse palynologique à l'étude des interactions sociétés-milieux, thèse de Doctorat, Université de Clermont-Ferrand, 368 p. [inédit].

PROVANSAL M., BERGER J.-F., BRAVARD J.-P., SALVADOR P.-G., ARNAUD-FASSETTA G., BRUNETON H., VÉROT-BOURRÉLY A

1999 : « Le régime du Rhône et les mutations des environnements fluviaux du lac de Genève à la mer ", in LEVEAU P. (DIR.), "Dossier: Le Rhône romain", Gallia, 56, p. 13-32.

\section{REILLE M.}

1990 : Lecons de palynologie et d'analyse pollinique, Paris, CNRS Éditions, 206 p.

SAVARON J.

1599: Sidonii Apollinaris Opera, Paris, A. Périer.

SIGAUT F.

1975 : L'Agriculture et le feu, Paris, éd. de la Maison des sciences de l'homme, $320 \mathrm{p}$.

\section{SYMÉONI G.}

1561 : Description de la Limagne d'Auvergne en forme de dialogue, avec plusieurs Médailles,
Statues, Oracles, Épitaphes, Sentences, et autres choses mémorables, et non moins plaisantes que proufitables aux amateurs de l'Antiquité, traduit du livre italien de Gabriel Syméoni (Dialogo pio e speculativo con diverse sentenze latine e volgari) en langue françoyse par Antoine Chappuys du Dauphiné (1560), Lyon, Guillaume Rouillé.

\section{TRÉMENT F.}

2002: «Le peuplement des campagnes d'Auvergne à l'époque romaine", in MARTIN D. (DIR.), L'Identité de l'Auvergne (Auvergne-Bourbonnais-Velay), mythe ou réalité historique? : essai sur une histoire de l'Auvergne des origines à nos jours, Nonette, éd. Créer, p. 83-112.

2004 : De la Gaule méditerranéenne à la Gaule centrale: paysages et peuplements à l'âge du Fer et à l'époque romaine. Archéologie et paléoenvironnement des campagnes de Provence et d'Auvergne, mémoire d'Habilitation à diriger les recherches, Aix-enProvence, Université de Provence, 295 p., 46 fig.

Trément F., ARgant J., BréHÉret JG., CAbanis M., Dousteyssier B., FOURMONT A., FOURNIER G., López Sáez J. A., Macaire J.-J., Prat B. VERNET G.

2005 : « Paysages et peuplement dans le bassin de Sarliève du Néolithique au Moyen Âge (Puy-de-Dôme, France) : éléments pour un nouveau modèle socio-environnemental », in BERGER J.-F., BERTONCELLO F., BRAEMER F., DAVTIAN G., GAZENBEEK M. (DIR.), Temps et espaces de l'homme en société : analyses et modèles spatiaux en archéologie, Antibes, APDCA, p. 485-498.

TRÉMENT F., BALlut C., DOUSTEYSSIER B., GUICHARD V., SEgaRd M.

2004: « Habitat et milieu humide en Grande Limagne de l'âge du Fer au Moyen Âge: essai de spatialisation dynamique des relations sociétés-milieux ", in Burnouf J., LeVEaU P. (DIR.), Fleuves et marais, une histoire au croisement de la nature et de la culture. Sociétés préindustrielles et milieux fluviaux, lacustres et palustres: pratiques sociales et hydrosystèmes, Paris, Comité des travaux historiques et scientifiques, p. 95-109.

\section{Trément F., Ballut C., Mennessier-} JOUANNET C., ARGANT J.

2002: "Mutations environnementales et systèmes socio-économiques en Grande Limagne (Massif central) de l'âge du Fer au Moyen Âge », in RICHARD H., 
VignOT A. (DIR.), Équilibres et ruptures dans les écosystèmes depuis 20000 ans en Europe de l'Ouest, Besançon (coll. Presses universitaires franc-comtoises, 831), p. 269-279.

\section{Trément F., Dousteyssier B.}

2003: "Élites et villae dans le territoire de la cité arverne", in CÉBEILLACGeRVASONI M., LAMOINE L. (DIR.), Les Élites et leurs facettes: les élites locales dans le monde hellénistique et romain, Rome, École française de Rome, Clermont-Ferrand, Presses universitaires Blaise-Pascal, p. $661-676$.

Trément F., Guichard V., MennessierJOUANNET C.

2002: "Aux origines de la cité arverne", in MARTIN D. (DIR.), L'Identité de l'Auvergne (Auvergne-Bourbonnais-Velay), mythe ou réalité historique? : essai sur une histoire de l'Auvergne des origines à nos jours, Nonette, éd. Créer, p. 166-193.

Trément F., Loison G., Argant J., BRÉHÉret J.-G., CABANIS M., DOUSTEYSSIER B., FOURMONT A., FOURNIER G., LIABEUF R., LóPEZ SÁEZ J. A., MACAIRE J.-J., MILCENT P.-Y., PRAT B., Rialland Y., VERNET G.

2006: «Interactions sociétés-milieux en Grande Limagne du Néolithique à l'époque romaine: apport des recherches interdisciplinaires conduites dans le bassin de Sarliève (Puy-de-Dôme) ", in FouÉrÉ P.,CHEVILlot C.,COURTAUd P.,
Ferullo O., Leroyer C. (DIR.), Paysages et peuplements. Aspects culturels et chronologiques en France méridionale: actualité de la recherche, Périgueux, coéd. Association pour le développement de la recherche archéologique et historique en PérigordPréhistoire du Sud-Ouest (coll. Suppl. à l'ADRAHP-PSO, 11), p. 11-32.

Trément F., MENNESSIER-JOUANNET C., ARGANT J., BRÉHÉRET J.-G., CABANIS M., DOUSTEYSSIER B., FOURMONT A., López Sáez J. A., Macaire J.-J., Prat B., VERNET G.

2007: «Le bassin de Sarliève: occupation du sol et paléo-environnement à l'âge du Fer ", in MENNESSIER-JOUANNET C., DEBERGE Y. (DIR.), L'Archéologie de l'âge du Fer en Auvergne: thème régional, Lattes, éd. Association pour le développement de l'archéologie en Languedoc-Roussillon (coll. Monographies d'archéologie méditerranéenne), p. 385-400.

TYSON R. V.

1995: Sedimentary Organic Matter (Organic Facies and Palynofacies), Londres, Chapman and Hall, 615 p.

VAN GEEL B., BUURMAN J., WATERBOLK H. T. 1996: "Archaeological and palaeoecological indications for an abrupt climate change in the Netherlands, and evidence for climatological teleconnections around $2650 \mathrm{BP}$ », Journal of Quaternary Science, 11, p. 451-460.

\section{VERGNE $V$.}

1989 : L'Évolution tardiglaciaire et holocène d'un piémont de moyenne montagne cristalline: l'Artense (Massif central, France), thèse de Doctorat, Université Paris-I-PanthéonSorbonne, 354 p. [inédit].

\section{VERGNETTE A.}

1927 : «La région de Sarliève, éclaircissements géologiques et historiques ", Revue d'Auvergne, 41, p. 257-269.

Vernet G., Caillat P., MennessierJouAnNet C., PARENT D., Rebiscoul A., RICHIER A., SÉriS D., VERNET B., WITTMANN A.

2005 : Rapport final d'opération de diagnostic et de fouille archéologique portant sur le bassin de Sarliève, Grande Halle d'Auvergne, INRAP, Direction interrégionale Rhône-Alpes/Auvergne, Centre archéologique régional d'Auvergne, 3 vol.

VERnET G., RAYNAL J.P.

2000: "Un cadre téphrostratigraphique réactualisé pour la préhistoire tardiglaciaire et holocène de Limagne (Massif central, France)", in Comptes rendus de l'Académie des sciences, 330, p. 309-405.

2002 : «Éruptions trachytiques de la chaîne des Puys (France) et leur impact sur les environnements", in RAYNAL J.-P., Albore-Livadie C., PIPERNO M. (DIR.), Hommes et volcans: de l'éruption à l'objet, Actes du symposium 15.2 du XIV congrès de l'Union internationale des sciences préhistoriques et protohistoriques, Liège, 2001, CDERAD (coll. Dossiers de l'Archéologis, 2), p. 49-56. 


\section{LISTE DES AUTEURS}

Jacqueline ARgAnt, Association de recherche paléoécologique en archéologie (ARPA), UFR des Sciences de la Terre, Université Lyon-I, Géode, 2 rue Raphaël-Dubois, F-69622 Villeurbanne Cedex. Courriel : j.argant@wanadoo.fr

Jean-Gabriel BRÉHÉRET, Laboratoire de géologie des environnements aquatiques continentaux (EA 2100-GéEAC), Faculté des sciences et techniques, Parc de Grandmont, F-37200 Tours. Courriel : jean-gabriel.breheret@univ-tours.fr

Manon CABANIS, Centre de recherche sur la préhistoire et la protohistoire de la Méditerranée (CRPPM, EHESS et UMR 5608 du CNRS), 39 allée Jules-Guesde, F-31000 Toulouse. Courriel : manon.cabanis@inrap.fr

Bertrand Dousteyssier, Centre d'histoire «Espaces et Cultures» (EA 1001-CHEC). Maison des sciences de l'homme, 4 rue Ledru, F-63057 Clermont-Ferrand Cedex 1. Courriel : bertrand.dousteyssier@univ-bpclermont.fr

Agathe Fourmont, Laboratoire de géologie des environnements aquatiques continentaux (EA 2100-GéEAC), Faculté des sciences et techniques, Parc de Grandmont, F-37200 Tours. Courriel : agathe.fourmont@hotmail.fr

Gabriel FOURNIER, ancien Professeur à l'Université Blaise-Pascal, 3 rue Adrien-Morin, F-63400 Chamalières.

René LiAbeUf, Service régional de l'archéologie, DRAC Auvergne, Hôtel de Chazerat, 4 rue Pascal, BP 378 , F-63010 Clermont-Ferrand Cedex 1. Courriel : rene.liabeuf@culture.gouv.fr

Gilles LOISON, Institut national de recherches archéologiques préventives (INRAP Méditerranée), 561 rue ÉtienneLenoir, km delta, F-39000 Nîmes. Courriel : gilles.loison@inrap.fr

José Antonio López SÁEz, Laboratorio de arqueobotánica, CEH, CSIC, Duque de Medinaceli 8, E-28014 Madrid. Courriel : cehl149@ceh.csic.es

Jean-Jacques MACAIRE, Laboratoire de géologie des environnements aquatiques continentaux (EA 2100-GéEAC), Faculté des sciences et techniques, Parc de Grandmont, F-37200 Tours. Courriel : jean-jacques.macaire@univ-tours.fr

Philippe MARINVAL, Centre de recherche sur la préhistoire et la protohistoire de la Méditerranée (CRPPM, EHESS et UMR 5608 du CNRS), 39 allée Jules-Guesde, F-31000 Toulouse. Courriel : marinval@cict.fr

Christine MENNESSIER-JOUANNET, Institut national de recherches archéologiques préventives (INRAP Rhône-AlpesAuvergne), 13 bis rue Pierre-Boulanger, F-63100 Clermont-Ferrand. Courriel : Chjouannet@aol.com

Pierre-Yves Milcent, Unité toulousaine d'archéologie et d'histoire (UMR 5608 du CNRS), 14 rue Cany, F-31300 Toulouse. Courriel : milcent@univ-tlse2.fr

Béatrice PRAT, Géolab (UMR 6042 du CNRS), Maison des sciences de l'homme, 4 rue Ledru, F-63057 Clermont-Ferrand Cedex 1. Courriel : beatriceprat@hotmail.com

Yannick RIALLAND, Service régional de l'archéologie, DRAC Auvergne, Hôtel de Chazerat, 4 rue Pascal, BP 378, F-63010 Clermont-Ferrand Cedex 1. Courriel : yannick.rialland@culture.gouv.fr

Frédéric Trément, Centre d'histoire «Espaces et Cultures » (EA 1001-CHEC). Maison des sciences de l'homme, 4 rue Ledru, F-63057 Clermont-Ferrand Cedex 1. Courriel : frederic.trement@wanadoo.fr

Gérard VERNET, Institut national de recherches archéologiques préventives (INRAP Rhône-Alpes-Auvergne), 13 bis rue Pierre-Boulanger, F-63100 Clermont-Ferrand. Courriel : gerard.vernet@inrap.fr 